\title{
AFFINE CELLULARITY OF KHOVANOV-LAUDA-ROUQUIER ALGEBRAS OF FINITE TYPES
}

\author{
ALEXANDER S. KLESHCHEV AND JOSEPH W. LOUBERT
}

\begin{abstract}
We prove that the Khovanov-Lauda-Rouquier algebras $R_{\alpha}$ of finite type are (graded) affine cellular in the sense of Koenig and Xi. In fact, we establish a stronger property, namely that the affine cell ideals in $R_{\alpha}$ are generated by idempotents. This in particular implies the (known) result that the global dimension of $R_{\alpha}$ is finite.
\end{abstract}

\section{INTRODUCTION}

The goal of this paper is to establish (graded) affine cellularity in the sense of Koenig and $\mathrm{Xi}$ [14] for the Khovanov-Lauda-Rouquier algebras $R_{\alpha}$ of finite Lie type. In fact, we construct a chain of affine cell ideals in $R_{\alpha}$ which are generated by idempotents. This stronger property is analogous to quasiheredity for finite dimensional algebras, and by a general result of Koenig and $\mathrm{Xi}$ [14, Theorem 4.4], it also implies finiteness of the global dimension of $R_{\alpha}$. Thus we obtain a new proof of (a slightly stronger version of) a recent result of Kato 6] and McNamara [19] (see also [2]). As another application, one gets a theory of standard and proper standard modules, cf. [6], [2]. It would be interesting to apply this paper to prove the conjectural (graded) cellularity of cyclotomic KLR algebras of finite types.

Our approach is independent of the homological results in [19], 6] and [2] (which relies on [19]). The connection between the theory developed in [2] and this paper is explained in [13]. This paper generalizes [10], where analogous results were obtained for finite type $A$.

We now give a definition of (graded) affine cellular algebra from 14, Definition 2.1]. Throughout the paper, unless otherwise stated, we assume that all algebras are $(\mathbb{Z})$-graded, all ideals, subspaces, etc. are homogeneous, and all homomorphisms are homogeneous degree zero homomorphisms with respect to the given gradings. For this introduction, we fix a noetherian domain $k$ (later on it will be sufficient to work with $k=\mathbb{Z}$ ). Let $A$ be a (graded) unital $k$-algebra with a $k$-anti-involution $\tau$. A (two-sided) ideal $J$ in $A$ is called an affine cell ideal if the following conditions are satisfied:

(1) $\tau(J)=J$

Research supported in part by the NSF grant no. DMS-1161094 and the Humboldt Foundation. Substrantial part of the paper has been completed at the University of Stuttgart. The authors thank Steffen Koenig for hospitality. 
(2) there exists an affine $k$-algebra $B$ with a $k$-involution $\sigma$ and a free $k$ module $V$ of finite rank such that $\Delta:=V \otimes_{k} B$ has an $A$ - $B$-bimodule structure, with the right $B$-module structure induced by the regular right $B$-module structure on $B$;

(3) let $\Delta^{\prime}:=B \otimes_{k} V$ be the $B$ - $A$-bimodule with left $B$-module structure induced by the regular left $B$-module structure on $B$ and right $A$ module structure defined by

$$
(b \otimes v) a=\mathrm{s}(\tau(a)(v \otimes b)),
$$

where s : $V \otimes_{k} B \rightarrow B \otimes_{k} V, v \otimes b \rightarrow b \otimes v$; then there is an $A$ $A$-bimodule isomorphism $\mu: J \rightarrow \Delta \otimes_{B} \Delta^{\prime}$, such that the following diagram commutes:

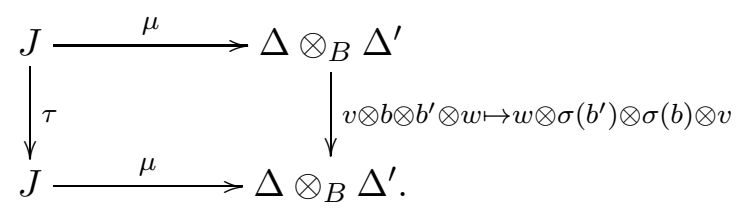

The algebra $A$ is called affine cellular if there is a $k$-module decomposition $A=J_{1}^{\prime} \oplus J_{2}^{\prime} \oplus \cdots \oplus J_{n}^{\prime}$ with $\tau\left(J_{l}^{\prime}\right)=J_{l}^{\prime}$ for $1 \leq l \leq n$, such that, setting $J_{m}:=\bigoplus_{l=1}^{m} J_{l}^{\prime}$, we obtain an ideal filtration

$$
0=J_{0} \subset J_{1} \subset J_{2} \subset \cdots \subset J_{n}=A
$$

so that each $J_{m} / J_{m-1}$ is an affine cell ideal of $A / J_{m-1}$.

To describe our main results we introduce some notation referring the reader to the main body of the paper for details. Fix a Cartan datum of finite type, and denote by $\Phi_{+}=\left\{\beta_{1}, \ldots, \beta_{N}\right\}$ the set of positive roots, and by $Q_{+}$the positive part of the root lattice. For $\alpha \in Q_{+}$we have the KLR algebra $R_{\alpha}$ with standard idempotents $\left\{e(\boldsymbol{i}) \mid \boldsymbol{i} \in\langle I\rangle_{\alpha}\right\}$. We denote by $\Pi(\alpha)$ the set of root partitions of $\alpha$. This is partially ordered with respect to a certain bilexicographic order ' $\leq$ '.

To any $\pi \in \Pi(\alpha)$ one associates a proper standard module $\bar{\Delta}(\pi)$ and a word $\boldsymbol{i}_{\pi} \in\langle I\rangle_{\alpha}$. We fix a distinguished vector $v_{\pi}^{+} \in \bar{\Delta}(\pi)$, and choose a set $\mathfrak{B}_{\pi} \subseteq R_{\alpha}$ so that $\left\{b v_{\pi}^{+} \mid b \in \mathfrak{B}_{\pi}\right\}$ is a basis of $\bar{\Delta}(\pi)$. We define polynomial subalgebras $\Lambda_{\pi} \subseteq R_{\alpha}$-these are isomorphic to tensor products of algebras of symmetric polynomials. We also explicitly define elements $\delta_{\pi}, D_{\pi} \in R_{\alpha}$ and set $e_{\pi}:=D_{\pi} \delta_{\pi}$. Then we set

$$
I_{\pi}^{\prime}:=k-\operatorname{span}\left\{b e_{\pi} \Lambda_{\pi} D_{\pi}\left(b^{\prime}\right)^{\tau} \mid b, b^{\prime} \in \mathfrak{B}_{\pi}\right\},
$$

$I_{\pi}:=\sum_{\sigma \geq \pi} I_{\sigma}^{\prime}$, and $I_{>\pi}=\sum_{\sigma>\pi} I_{\sigma}^{\prime}$. Our main results are now as follows

Main Theorem. The algebra $R_{\alpha}$ is graded affine cellular with cell chain given by the ideals $\left\{I_{\pi} \mid \pi \in \Pi(\alpha)\right\}$. Moreover, for a fixed $\pi \in \Pi(\alpha)$, we set $\bar{R}_{\alpha}:=R_{\alpha} / I_{>\pi}$ and $\bar{h}:=h+I_{>\pi}$ for any $h \in R_{\alpha}$. We have:

(i) $I_{\pi}=\sum_{\sigma \geq \pi} R_{\alpha} e\left(\boldsymbol{i}_{\sigma}\right) R_{\alpha}$;

(ii) $\bar{e}_{\pi}$ is an idempotent in $\bar{R}_{\alpha}$; 
(iii) the map $\Lambda_{\pi} \rightarrow \bar{e}_{\pi} \bar{R}_{\alpha} \bar{e}_{\pi}, f \mapsto \bar{e}_{\pi} \bar{f} \bar{e}_{\pi}$ is an isomorphism of graded algebras;

(iv) $\bar{R}_{\alpha} \bar{e}_{\pi}$ is a free right $\bar{e}_{\pi} \bar{R}_{\alpha} \bar{e}_{\pi}$-module with basis $\left\{\bar{b} \bar{e}_{\pi} \mid b \in \mathfrak{B}_{\pi}\right\}$;

(v) $\bar{e}_{\pi} \bar{R}_{\alpha}$ is a free left $\bar{e}_{\pi} \bar{R}_{\alpha} \bar{e}_{\pi}$-module with basis $\left\{\bar{e}_{\pi} \bar{D}_{\pi} \bar{b}^{\tau} \mid b \in \mathfrak{B}_{\pi}\right\}$;

(vi) multiplication provides an isomorphism

$$
\bar{R}_{\alpha} \bar{e}_{\pi} \otimes_{\bar{e}_{\pi} \bar{R}_{\alpha} \bar{e}_{\pi}} \bar{e}_{\pi} \bar{R}_{\alpha} \stackrel{\sim}{\longrightarrow} \bar{R}_{\alpha} \bar{e}_{\pi} \bar{R}_{\alpha}
$$

(vii) $\bar{R}_{\alpha} \bar{e}_{\pi} \bar{R}_{\alpha}=I_{\pi} / I_{>\pi}$.

Main Theorem(vii) shows that each affine cell ideal $I_{\pi} / I_{>\pi}$ in $R_{\alpha} / I_{>\pi}$ is generated by an idempotent. This, together with the fact that each algebra $\Lambda_{\pi}$ is a polynomial algebra, is enough to invoke [14, Theorem 4.4] to get

Corollary. If the ground ring $k$ has finite global dimension, then the algebra $R_{\alpha}$ has finite global dimension.

This seems to be a slight generalization of [6], [19], 2] in two ways: 6] assumes that $k$ is a field of characteristic zero (and the Lie type is simplylaced), while [19, [2] assume that $k$ is a field; moreover, [6], [19], 2] deal with categories of graded modules only, while our corollary holds for the algebra $R_{\alpha}$ even as an ungraded algebra.

The paper is organized as follows. Section 2 contains preliminaries needed for the rest of the paper. The first subsection contains mostly general conventions that will be used. Subsection 2.2 goes over the Lie theoretic notation that we employ. We move on in subsection 2.3 to the definition and basic results of Khovanov-Lauda-Rouquier (KLR) algebras. The next two subsections are devoted to recalling results about the representation theory of KLR algebras. Then, in subsection 2.6, we introduce our notation regarding quantum groups, and recall some well-known basis theorems. The next subsection is devoted to the connection between KLR algebras and quantum groups, namely the categorification theorems. Finally, subsection 2.8 contains an easy direct proof of a graded dimension formula for the KLR algebras, cf. [2, Corollary 3.15].

Section 3 is devoted to constructing a basis for the KLR algebras that is amenable to checking affine cellularity. We begin in subsection 3.1 by choosing some special weight idempotents and proving some properties they enjoy. Subsection 3.2 introduces the notation that allows us to define our affine cellular structure. This subsection also contains the crucial Hypothesis 3.9. Next, in subsection 3.3, we come up with an affine cellular basis in the special case corresponding to a root partition of that is a power of a single root. Finally, we use this in the last subsection to come up with our affine cellular basis in full generality.

In section 4 we show how the affine cellular basis is used to prove that the KLR algebras are affine cellular.

Finally, in section 5 we verify Hypothesis 3.9 for all positive roots in all finite types. We begin in subsection 5.1 by recalling some results concerning homogeneous representations. In subsection 5.2 we recall the definition 
of special Lyndon orders and Lyndon words, which will serve as the special weights of subsection 3.1. The next subsection is devoted to verifying Hypothesis 3.9 in the special case when the cuspidal representation corresponding to the positive root is homogeneous. We then employ this in subsection 5.4 to show that the hypothesis holds in simply-laced types. Finally, we have subsection 5.5. wherein we verify the hypothesis by hand in the non-symmetric types.

\section{Preliminaries AND A Dimension formula}

In this section we set up the theory of KLR algebras and their connection to quantum groups following mainly [7] and also [1]. Only subsection 2.8 contains some new material.

2.1. Generalities. Throughout the paper we work over the ground ring $\mathcal{O}$ which is assumed to be either $\mathbb{Z}$ or an arbitrary field $F$. Most of the time we work over $F$ and then deduce the corresponding result for $\mathbb{Z}$ using the following standard lemma

Lemma 2.1. Let $M$ be a finitely generated $\mathbb{Z}$-module, and $\left\{x_{\alpha}\right\}_{\alpha \in A}$ a subset of $M$. Then $\left\{x_{\alpha}\right\}$ is a spanning set (resp. basis) of $M$ if and only if $\left\{1_{F} \otimes x_{\alpha}\right\}$ is a spanning set (resp. basis) of $F \otimes_{\mathbb{Z}} M$ for every field $F$.

Let $q$ be an indeterminate, $\mathbb{Q}(q)$ the field of rational functions, and $\mathcal{A}:=$ $\mathbb{Z}\left[q, q^{-1}\right] \subseteq \mathbb{Q}(q)$. Let ${ }^{-}: \mathbb{Q}(q) \rightarrow \mathbb{Q}(q)$ be the $\mathbb{Q}$-algebra involution with $\bar{q}=q^{-1}$, referred to as the bar-involution.

For a graded vector space $V=\oplus_{n \in \mathbb{Z}} V_{n}$, with finite dimensional graded components its graded dimension is $\operatorname{dim}_{q} V:=\sum_{n \in \mathbb{Z}}\left(\operatorname{dim} V_{n}\right) q^{n} \in \mathbb{Z}\left[\left[q, q^{-1}\right]\right]$. For any graded $F$-algebra $H$ we denote by $H$-Mod the abelian category of all graded left $H$-modules, with morphisms being degree-preserving module homomorphisms, which we denote by hom. Let $H$-mod denote the abelian subcategory of all finite dimensional graded $H$-modules and $H$-proj denote the additive subcategory of all finitely generated projective graded $H$-modules. Denote the corresponding Grothendieck groups by $[H$-mod] and $[H$-proj], respectively. These Grothendieck groups are $\mathcal{A}$-modules via $q^{m}[M]:=[M\langle m\rangle]$, where $M\langle m\rangle$ denotes the module obtained by shifting the grading up by $m: M\langle m\rangle_{n}:=M_{n-m}$. For $n \in \mathbb{Z}$, let $\operatorname{Hom}_{H}(M, N)_{n}:=$ $\operatorname{hom}_{H}(M\langle n\rangle, N)$ denote the space of homomorphisms of degree $n$. Set $\operatorname{Hom}_{H}(M, N):=\bigoplus_{n \in \mathbb{Z}} \operatorname{Hom}_{H}(M, N)_{n}$.

2.2. Lie theoretic data. A Cartan datum is a pair $(I, \cdot)$ consisting of a set $I$ and a $\mathbb{Z}$-valued symmetric bilinear form $i, j \mapsto i \cdot j$ on the free abelian group $\mathbb{Z}[I]$ such that $i \cdot i \in\{2,4,6, \ldots\}$ for all $i \in I$ and $2(i \cdot j) /(i \cdot i) \in\{0,-1,-2 \ldots\}$ for all $i \neq j$ in $I$. Set $a_{i j}:=2(i \cdot j) /(i \cdot i)$ for $i, j \in I$ and define the Cartan matrix $A:=\left(a_{i j}\right)_{i, j \in I}$. Throughout the paper, unless otherwise stated, we assume that $A$ has finite type, see [5, §4]. We have simple roots $\left\{\alpha_{i} \mid i \in I\right\}$, and we identify $\alpha_{i}$ with $i$. Let $Q_{+}:=\bigoplus_{i \in I} \mathbb{Z}_{\geq 0} \alpha_{i}$. For $\alpha \in Q_{+}$, we write $h t(\alpha)$ for the sum of its coefficients when expanded in terms of the 
$\alpha_{i}$ 's. Denote by $\Phi_{+} \subset Q_{+}$the set of positive roots, cf. [5, $\left.\S 1.3\right]$, and by $W$ the corresponding Weyl group. A total order on $\Phi_{+}$is called convex if $\beta, \gamma, \beta+\gamma \in \Phi_{+}$and $\beta<\gamma$ imply $\beta<\beta+\gamma<\gamma$.

Given $\beta \in \mathbb{Z}[I]$, denote

$$
q_{\beta}:=q^{(\beta \cdot \beta) / 2},[n]_{\beta}:=\left(q_{\beta}^{n}-q_{\beta}^{-n}\right) /\left(q_{\beta}-q_{\beta}^{-1}\right),[n]_{\beta}^{!}:=[n]_{\beta}[n-1]_{\beta} \ldots[1]_{\beta} .
$$

In particular, for $i \in I$, we have $q_{i},[n]_{i},[n]_{i}^{!}$. Let $A$ be a $Q_{+}$-graded $\mathbb{Q}(q)$ algebra, $\theta \in A_{\alpha}$ for $\alpha \in Q_{+}$, and $n \in \mathbb{Z}_{>0}$. We use the standard notation for quantum divided powers: $\theta^{(n)}:=\theta^{n} /[n]_{\alpha}^{!}$.

Denote by $\langle I\rangle:=\bigsqcup_{d \geq 0} I^{d}$ the set of all tuples $\boldsymbol{i}=i_{1} \ldots i_{d}$ of elements of $I$, which we refer to as words. We consider $\langle I\rangle$ as a monoid under the concatenation product. If $\boldsymbol{i} \in\langle I\rangle$, we can write it in the form $\boldsymbol{i}=j_{1}^{m_{1}} \ldots j_{r}^{m_{r}}$ for $j_{1}, \ldots, j_{r} \in I$ such that $j_{s} \neq j_{s+1}$ for all $s=1,2, \ldots, r-1$. We then denote

$$
[\boldsymbol{i}] !:=\left[m_{1}\right]_{j_{1}}^{!} \ldots\left[m_{r}\right]_{j_{r}}^{!} .
$$

For $\boldsymbol{i}=i_{1} \ldots i_{d}$ set $|\boldsymbol{i}|:=\alpha_{i_{1}}+\cdots+\alpha_{i_{d}} \in Q_{+}$. The symmetric group $S_{d}$ with simple transpositions $s_{1}, \ldots, s_{d-1}$ acts on $I^{d}$ on the left by place

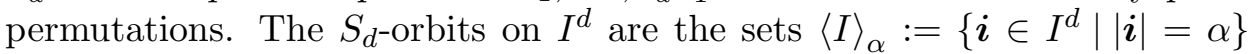
parametrized by the elements $\alpha \in Q_{+}$of height $d$.

2.3. Khovanov-Lauda-Rouquier algebras. Let $A$ be a Cartan matrix. Choose signs $\varepsilon_{i j}$ for all $i, j \in I$ with $a_{i j}<0$ so that $\varepsilon_{i j} \varepsilon_{j i}=-1$, and define the polynomials $\left\{Q_{i j}(u, v) \in F[u, v] \mid i, j \in I\right\}$ :

$$
Q_{i j}(u, v):= \begin{cases}0 & \text { if } i=j ; \\ 1 & \text { if } a_{i j}=0 \\ \varepsilon_{i j}\left(u^{-a_{i j}}-v^{-a_{j i}}\right) & \text { if } a_{i j}<0 .\end{cases}
$$

In addition, fix $\alpha \in Q_{+}$of height $d$. Let $R_{\alpha}=R_{\alpha}(\Gamma, \mathcal{O})$ be an associative graded unital $\mathcal{O}$-algebra, given by the generators

$$
\left\{e(\boldsymbol{i}) \mid \boldsymbol{i} \in\langle I\rangle_{\alpha}\right\} \cup\left\{y_{1}, \ldots, y_{d}\right\} \cup\left\{\psi_{1}, \ldots, \psi_{d-1}\right\}
$$

and the following relations for all $\boldsymbol{i}, \boldsymbol{j} \in\langle I\rangle_{\alpha}$ and all admissible $r, t$ :

$$
\begin{gathered}
e(\boldsymbol{i}) e(\boldsymbol{j})=\delta_{\boldsymbol{i}, \boldsymbol{j}} e(\boldsymbol{i}), \quad \sum_{\boldsymbol{i} \in\langle I\rangle_{\alpha}} e(\boldsymbol{i})=1 ; \\
y_{r} e(\boldsymbol{i})=e(\boldsymbol{i}) y_{r} ; \quad y_{r} y_{t}=y_{t} y_{r} ; \\
\psi_{r} e(\boldsymbol{i})=e\left(s_{r} \boldsymbol{i}\right) \psi_{r} \\
y_{r} \psi_{s}=\psi_{s} y_{r} \quad(r \neq s, s+1) \\
\left(y_{t} \psi_{r}-\psi_{r} y_{s_{r}(t)}\right) e(\boldsymbol{i})=\delta_{i_{r}, i_{r+1}}\left(\delta_{t, r+1}-\delta_{t, r}\right) e(\boldsymbol{i}) ; \\
\psi_{r}^{2} e(\boldsymbol{i})=Q_{i_{r}, i_{r+1}}\left(y_{r}, y_{r+1}\right) e(\boldsymbol{i}) \\
\psi_{r} \psi_{t}=\psi_{t} \psi_{r} \quad(|r-t|>1) ; \\
\left(\psi_{r+1} \psi_{r} \psi_{r+1}-\psi_{r} \psi_{r+1} \psi_{r}\right) e(\boldsymbol{i}) \\
=\delta_{i_{r}, i_{r+2}} \frac{Q_{i_{r}, i_{r+1}}\left(y_{r+2}, y_{r+1}\right)-Q_{i_{r}, i_{r+1}}\left(y_{r}, y_{r+1}\right)}{y_{r+2}-y_{r}} e(\boldsymbol{i}) .
\end{gathered}
$$


The grading on $R_{\alpha}$ is defined by setting:

$$
\operatorname{deg}(e(\boldsymbol{i}))=0, \quad \operatorname{deg}\left(y_{r} e(\boldsymbol{i})\right)=i_{r} \cdot i_{r}, \quad \operatorname{deg}\left(\psi_{r} e(\boldsymbol{i})\right)=-i_{r} \cdot i_{r+1} .
$$

In this paper grading always means $\mathbb{Z}$-grading, ideals are assumed to be homogeneous, and modules are assumed graded, unless otherwise stated.

It is pointed out in [8] and [20, §3.2.4] that up to isomorphism the graded $\mathcal{O}$-algebra $R_{\alpha}$ depends only on the Cartan datum and $\alpha$. We refer to the algebra $R_{\alpha}$ as an (affine) Khovanov-Lauda-Rouquier algebra. It is convenient to consider the direct sum of algebras $R:=\bigoplus_{\alpha_{\in} Q_{+}} R_{\alpha}$. Note that $R$ is nonunital, but it is locally unital since each $R_{\alpha}$ is unital. The algebra $R_{\alpha}$ possesses a graded anti-automorphism

$$
\tau: R_{\alpha} \rightarrow R_{\alpha}, x \mapsto x^{\tau}
$$

which is the identity on generators.

For each element $w \in S_{d}$ fix a reduced expression $w=s_{r_{1}} \ldots s_{r_{m}}$ and set $\psi_{w}:=\psi_{r_{1}} \ldots \psi_{r_{m}}$. In general, $\psi_{w}$ depends on the choice of the reduced expression of $w$.

Theorem 2.2. [7, Theorem 2.5], [20, Theorem 3.7] The following set is an $\mathcal{O}$-basis of $R_{\alpha}:\left\{\psi_{w} y_{1}^{m_{1}} \ldots y_{d}^{m_{d}} e(\boldsymbol{i}) \mid w \in S_{d}, m_{1}, \ldots, m_{d} \in \mathbb{Z}_{\geq 0}, \boldsymbol{i} \in\langle I\rangle_{\alpha}\right\}$.

In view of the theorem, we have a polynomial subalgebra

$$
P_{d}=\mathcal{O}\left[y_{1}, \ldots, y_{d}\right] \subseteq R_{\alpha}
$$

Let $\gamma_{1}, \ldots, \gamma_{l}$ be elements of $Q_{+}$with $\gamma_{1}+\cdots+\gamma_{l}=\alpha$. Then we have a natural embedding

$$
\iota_{\gamma_{1}, \ldots, \gamma_{l}}: R_{\gamma_{1}} \otimes \cdots \otimes R_{\gamma_{l}} \hookrightarrow R_{\alpha}
$$

of algebras, whose image is the parabolic subalgebra $R_{\gamma_{1}, \ldots, \gamma_{l}} \subseteq R_{\alpha}$. This is not a unital subalgebra, the image of the identity element of $R_{\gamma_{1}} \otimes \cdots \otimes R_{\gamma_{l}}$ being

$$
1_{\gamma_{1}, \ldots, \gamma_{l}}=\sum_{\boldsymbol{i}^{(1)} \in\langle I\rangle_{\gamma_{1}}, \ldots, \boldsymbol{i}^{(l)} \in\langle I\rangle_{\gamma_{l}}} e\left(\boldsymbol{i}^{(1)} \ldots \boldsymbol{i}^{(l)}\right) .
$$

An important special case is where $\alpha=d \alpha_{i}$ is a multiple of a simple root, in which case we have that $R_{d \alpha_{i}}$ is the $d^{\text {th }}$ nilHecke algebra $H_{d}$ generated by $\left\{y_{1}, \ldots, y_{d}, \psi_{1}, \ldots, \psi_{d-1}\right\}$ subject to the relations

$$
\begin{aligned}
\psi_{r}^{2} & =0 \\
\psi_{r} \psi_{s} & =\psi_{s} \psi_{r} \quad \text { if }|r-s|>1 \\
\psi_{r} \psi_{r+1} \psi_{r} & =\psi_{r+1} \psi_{r} \psi_{r+1} \\
\psi_{r} y_{s} & =y_{s} \psi_{r} \quad \text { if } s \neq r, r+1 \\
\psi_{r} y_{r+1} & =y_{r} \psi_{r}+1 \\
y_{r+1} \psi_{r} & =\psi_{r} y_{r}+1 .
\end{aligned}
$$

The grading is so that $\operatorname{deg}\left(y_{r}\right)=\alpha_{i} \cdot \alpha_{i}$ and $\operatorname{deg}\left(\psi_{r}\right)=-\alpha_{i} \cdot \alpha_{i}$. Note that here the elements $\psi_{w}$ do not depend on a choice of reduced decompositions. 
Let $w_{0} \in \mathfrak{S}_{d}$ be the longest element, and define the following elements of $H_{d}$ :

It is known that

$$
\delta_{d}:=y_{2} y_{3}^{2} \ldots y_{d}^{d-1}, \quad e_{d}:=\psi_{w_{0}} \delta_{d}
$$

$$
e_{d} \psi_{w_{0}}=\psi_{w_{0}}
$$

and in particular $e_{d}$ is an idempotent, see for example [7, §2.2]. The following is a special case of our main theorem for the case where $\alpha=d \alpha_{i}$, which will be used in its proof. It is known that the center $Z\left(H_{d}\right)$ consists of the symmetric polynomials $\mathcal{O}\left[y_{1}, \ldots, y_{d}\right]^{\mathfrak{S}_{d}}$.

Theorem 2.3. [10, Theorem 4.16] Let $X$ be a $\mathcal{O}$-basis of $\mathcal{O}\left[y_{1}, \ldots, y_{d}\right]^{\mathfrak{S}_{d}}$ and let $\mathfrak{B}$ be a basis of $\mathcal{O}\left[y_{1}, \ldots, y_{d}\right]$ as an $\mathcal{O}\left[y_{1}, \ldots, y_{d}\right]^{\mathfrak{S}_{d}}$-module. Then $\left\{b e_{d} f \psi_{w_{0}}\left(b^{\prime}\right)^{\tau} \mid b, b^{\prime} \in \mathfrak{B}, f \in X\right\}$ is a $\mathcal{O}$-basis of $H_{d}$.

2.4. Basic representation theory of $R_{\alpha}$. By [7], every irreducible graded $R_{\alpha}$-module is finite dimensional, and there are finitely many irreducible $R_{\alpha^{-}}$ modules up to isomorphism and grading shift. For $\boldsymbol{i} \in\langle I\rangle_{\alpha}$ and $M \in$ $R_{\alpha}$-Mod, the $\boldsymbol{i}$-word space of $M$ is $M_{\boldsymbol{i}}:=e(\boldsymbol{i}) M$. We have a decomposition of (graded) vector spaces $M=\bigoplus_{\boldsymbol{i} \in\langle I\rangle_{\alpha}} M_{\boldsymbol{i}}$. We say that $\boldsymbol{i}$ is a word of $M$ if $M_{i} \neq 0$.

We identify in a natural way:

$$
[R-\mathrm{mod}]=\bigoplus_{\alpha \in Q_{+}}\left[R_{\alpha} \text {-mod }\right], \quad[R-\text { proj }]=\bigoplus_{\alpha \in Q_{+}}\left[R_{\alpha}-\text { proj }\right] .
$$

Recall the anti-automorphism $\tau$ from (2.11). This allows us to introduce the left $R_{\alpha}$-module structure on the graded dual of a finite dimensional $R_{\alpha}$-module $M$-the resulting left $R_{\alpha}$-module is denoted $M^{\circledast}$. On the other hand, given any left $R_{\alpha}$-module $M$, denote by $M^{\tau}$ the right $R_{\alpha}$-module with the action given by $m x=\tau(x) m$ for $x \in R_{\alpha}, m \in M$. Following [8, (14)], define the Khovanov-Lauda pairing to be the $\mathcal{A}$-linear pairing

$$
(\cdot, \cdot):\left[R_{\alpha} \text {-proj }\right] \times\left[R_{\alpha} \text {-proj }\right] \rightarrow \mathcal{A} \cdot \prod_{i \in I} \prod_{a=1}^{m_{i}} \frac{1}{\left(1-q_{i}^{2 a}\right)}
$$

such that $([P],[Q])=\operatorname{dim}_{q}\left(P^{\tau} \otimes_{R_{\alpha}} Q\right)$.

Let $\alpha, \beta \in Q_{+}$. Recalling the isomorphism $\iota_{\alpha, \beta}: R_{\alpha} \otimes R_{\beta} \rightarrow R_{\alpha, \beta} \subseteq$ $R_{\alpha+\beta}$, consider the functors

$$
\begin{aligned}
& \operatorname{Ind}_{\alpha, \beta}:=R_{\alpha+\beta} 1_{\alpha, \beta} \otimes_{R_{\alpha, \beta}} ?: R_{\alpha, \beta}-\operatorname{Mod} \rightarrow R_{\alpha+\beta} \text {-Mod, } \\
& \operatorname{Res}_{\alpha, \beta}:=1_{\alpha, \beta} R_{\alpha+\beta} \otimes_{\alpha+\beta} ?: R_{\alpha+\beta} \text { Mod } \rightarrow R_{\alpha, \beta}-\operatorname{Mod} .
\end{aligned}
$$

For $M \in R_{\alpha}$-mod and $N \in R_{\beta}$-mod, we denote $M \circ N:=\operatorname{Ind}_{\alpha, \beta}(M \otimes N)$. The functors of induction define products on the Grothendieck groups $[R$-mod] and $[R$-proj] and the functors of restriction define coproducts on $[R$-mod] and $[R$-proj]. These products and coproducts make $[R$-mod $]$ and $[R$-proj $]$ into twisted unital and counital bialgebras [7, Proposition 3.2]. 
Let $i \in I$ and $n \in \mathbb{Z}_{>0}$. As explained in [7, §2.2], the algebra $R_{n \alpha_{i}}$ has a representation on the polynomials $F\left[y_{1}, \ldots, y_{n}\right]$ such that each $y_{r}$ acts as multiplication by $y_{r}$ and each $\psi_{r}$ acts as the divided difference operator $\partial_{r}: f \mapsto \frac{s_{r} f-f}{y_{r}-y_{r+1}}$. Let $P\left(i^{(n)}\right)$ denote this representation of $R_{n \alpha_{i}}$ viewed as a graded $R_{n \alpha_{i}}$-module with grading defined by

$$
\operatorname{deg}\left(y_{1}^{m_{1}} \cdots y_{n}^{m_{n}}\right):=\left(\alpha_{i} \cdot \alpha_{i}\right)\left(m_{1}+\cdots+m_{n}-n(n-1) / 4\right) .
$$

By [7, §2.2], the left regular $R_{n \alpha_{i}}$-module decomposes as $P\left(i^{n}\right) \cong[n]_{i}^{!} \cdot P\left(i^{(n)}\right)$. In particular, $P\left(i^{(n)}\right)$ is projective. Set

$$
\begin{aligned}
\theta_{i}^{(n)} & :=\operatorname{Ind}_{\alpha, n \alpha_{i}}\left(? \otimes P\left(i^{(n)}\right)\right): R_{\alpha^{-}} \text {Mod } \rightarrow R_{\alpha+n \alpha_{i}}-\operatorname{Mod}, \\
\left(\theta_{i}^{*}\right)^{(n)} & :=\operatorname{Hom}_{R_{n \alpha_{i}}^{\prime}}\left(P\left(i^{(n)}\right), ?\right): R_{\alpha+n \alpha_{i}}-\operatorname{Mod} \rightarrow R_{\alpha}-\operatorname{Mod},
\end{aligned}
$$

where $R_{n \alpha_{i}}^{\prime}:=1 \otimes R_{n \alpha_{i}} \subseteq R_{\alpha, n \alpha_{i}}$. These functors induce $\mathcal{A}$-linear maps on the corresponding Grothendieck groups:

$$
\theta_{i}^{(n)}:\left[R_{\alpha} \text {-proj] } \rightarrow\left[R_{\alpha+n \alpha_{i}}-\text { proj }\right], \quad\left(\theta_{i}^{*}\right)^{(n)}:\left[R_{\alpha+n \alpha_{i}}-\bmod \right] \rightarrow\left[R_{\alpha}-\bmod \right] .\right.
$$

2.5. Cuspidal and standard modules. Standard module theory for $R_{\alpha}$ has been developed in $3,4,11,19$. Here we follow the most general approach of McNamara [19]. Fix a reduced decomposition $w_{0}=s_{i_{1}} \ldots s_{i_{N}}$ of the longest element $w_{0} \in W$. This gives a convex total order on the positive roots

$$
\Phi_{+}=\left\{\beta_{1}>\cdots>\beta_{N}\right\},
$$

with $\beta_{N+1-k}=s_{i_{1}} \ldots s_{i_{k-1}}\left(\alpha_{i_{k}}\right)$.

To every positive root $\beta \in \Phi_{+}$of the corresponding root system $\Phi$, one associates a cuspidal module $L(\beta)$. This irreducible module is uniquely determined by the following property: if $\delta, \gamma \in Q_{+}$are non-zero elements such that $\beta=\delta+\gamma$ and $\operatorname{Res}_{\delta, \gamma} L(\beta) \neq 0$, then $\delta$ is a sum of positive roots less than $\beta$ and $\gamma$ is a sum of positive roots greater than $\beta$.

A standard argument involving the Mackey Theorem from [7] and convexity as in the proof of [2, Lemma 2.11], yields:

Lemma 2.4. Let $\beta \in \Phi_{+}$and $a_{1}, \ldots, a_{n} \in \mathbb{Z}_{\geq 0}$. All composition factors of $\operatorname{Res}_{a_{1} \beta, \ldots, a_{n} \beta} L(\beta)^{\circ\left(a_{1}+\cdots+a_{n}\right)}$ are of the form $L(\beta)^{\circ a_{1}} \nabla \cdots \nabla L(\beta)^{\circ a_{n}}$.

Let $\alpha \in Q_{+}$. A tuple $\pi=\left(p_{1}, \ldots p_{N}\right) \in \mathbb{Z}_{\geq 0}^{N}$ is called a root partition of $\alpha$ if $p_{1} \beta_{1}+\cdots+p_{N} \beta_{N}=\alpha$. We also use the notation $\pi=\left(\beta_{1}^{p_{1}}, \ldots, \beta_{N}^{p_{N}}\right)$. For example, if $\alpha=n \beta$ for $\beta \in \Phi_{+}$, we have a root partition $\left(\beta^{n}\right) \in \Pi(\alpha)$. Denote by $\Pi(\alpha)$ the set of all root partitions of $\alpha$. This set has two total orders: $\leq_{l}$ and $\leq_{r}$ defined as follows: $\left(p_{1}, \ldots, p_{N}\right)<_{l}\left(s_{1}, \ldots, s_{N}\right)$ (resp. $\left.\left(p_{1}, \ldots, p_{N}\right)<_{r}\left(s_{1}, \ldots, s_{N}\right)\right)$ if there exists $1 \leq k \leq N$ such that $p_{k}<s_{k}$ and $p_{m}=s_{m}$ for all $m<k$ (resp. $m>k$ ). Finally, we have a bilexicographic partial order:

$$
\pi \leq \sigma \Longleftrightarrow \pi \leq_{l} \sigma \text { and } \pi \leq_{r} \sigma \quad(\pi, \sigma \in \Pi(\alpha)) .
$$

The following lemma is implicit in [19]; see also [2, Lemma 2.5]. 
Lemma 2.5. Given any $\pi \in \Pi(p \beta)$, we have $\pi \geq\left(\beta^{p}\right)$.

For a root partition $\pi=\left(p_{1}, \ldots, p_{N}\right) \in \Pi(\alpha)$ as above, set $\operatorname{sh}(\pi):=$ $\sum_{k=1}^{N}\left(\beta_{k} \cdot \beta_{k}\right) p_{k}\left(p_{k}-1\right) / 4$, and define the corresponding proper standard module

$$
\bar{\Delta}(\pi):=L\left(\beta_{1}\right)^{\circ p_{1}} \circ \cdots \circ L\left(\beta_{N}\right)^{\circ p_{N}}\langle\operatorname{sh}(\pi)\rangle .
$$

For $\pi=\left(\beta_{1}^{p_{1}}, \ldots, \beta_{N}^{p_{N}}\right)$, we denote

$$
\operatorname{Res}_{\pi}:=\operatorname{Res}_{p_{1} \beta_{1}, \ldots, p_{N} \beta_{N}} .
$$

Theorem 2.6. [19] For any convex order there exists a cuspidal system $\left\{L(\beta) \mid \beta \in \Phi_{+}\right\}$. Moreover:

(i) For every $\pi \in \Pi(\alpha)$, the proper standard module $\bar{\Delta}(\pi)$ has irreducible head; denote this irreducible module $L(\pi)$.

(ii) $\{L(\pi) \mid \pi \in \Pi(\alpha)\}$ is a complete and irredundant system of irreducible $R_{\alpha}$-modules up to isomorphism.

(iii) $L(\pi)^{\circledast} \simeq L(\pi)$.

(iv) $[\bar{\Delta}(\pi): L(\pi)]_{q}=1$, and $[\bar{\Delta}(\pi): L(\sigma)]_{q} \neq 0$ implies $\sigma \leq \pi$.

(v) $L(\beta)^{\text {on }}$ is irreducible for every $\beta \in \Phi_{+}$and every $n \in \mathbb{Z}_{>0}$.

(vi) $\operatorname{Res}_{\pi} \bar{\Delta}(\sigma) \neq 0$ implies $\sigma \geq \pi$, and $\operatorname{Res}_{\pi} \bar{\Delta}(\pi) \simeq L\left(\beta_{1}\right)^{\circ p_{1}} \otimes \cdots \otimes$ $L\left(\beta_{N}\right)^{\circ p_{N}}$.

Note that the algebra $R_{\alpha}(F)$ is defined over $\mathbb{Z}$, i.e. $R_{\alpha}(F) \simeq R_{\alpha}(\mathbb{Z}) \otimes_{\mathbb{Z}} F$. We will use the corresponding indices when we need to distinguish between modules defined over different rings. The following result shows that cuspidal modules are also defined over $\mathbb{Z}$ :

Lemma 2.7. Let $\beta \in \Phi_{+}$, and $v \in L(\beta)_{\mathbb{Q}}$ be a non-zero homogeneous vector. Then $L(\beta)_{\mathbb{Z}}:=R_{\beta}(\mathbb{Z}) \cdot v \subset L(\beta)_{\mathbb{Q}}$ is an $R_{\beta}(\mathbb{Z})$-invariant lattice such that $L(\beta)_{\mathbb{Z}} \otimes_{\mathbb{Z}} F \simeq L(\beta)_{F}$ as $R_{\beta}(F)$-modules for any field $F$.

Proof. Note using degrees that $L(\beta)_{\mathbb{Z}}$ is finitely generated over $\mathbb{Z}$, hence it is a lattice in $L(\beta)_{\mathbb{Q}}$. Furthermore $\operatorname{ch}_{q} L(\beta)_{\mathbb{Z}} \otimes_{\mathbb{Z}} F=\operatorname{ch}_{q} L(\beta)_{\mathbb{Q}}$, whence by definition of the cuspidal modules, all composition factors of $\operatorname{ch}_{q} L(\beta)_{\mathbb{Z}} \otimes_{\mathbb{Z}} F$ are of the form $L(\beta)_{F}$. But there is always a multiplicity one composition factor in a reduction modulo $p$ of any irreducible module over a KLR algebra, thank to [9, Lemma 4.7].

2.6. Quantum groups. Following [17, Section 1.2], we define the algebra 'f to be the free $\mathbb{Q}(q)$-algebra with generators ' $\theta_{i}$ for $i \in I$ (our $q$ is Lusztig's $v^{-1}$, in keeping with the conventions of [7]). This algebra is $Q_{+}$-graded by assigning the degree $\alpha_{i}$ to ${ }^{\prime} \theta_{i}$ for each $i \in I$, so that ${ }^{\prime} \mathbf{f}=\oplus_{\alpha \in Q_{+}}{ }^{\prime} \mathbf{f}_{\alpha}$. If $x \in{ }^{\prime} \mathbf{f}_{\alpha}$, we write $|x|=\alpha$. For $\boldsymbol{i}=\left(i_{1}, \ldots, i_{n}\right) \in\langle I\rangle$, write ${ }^{\prime} \theta_{\boldsymbol{i}}:={ }^{\prime} \theta_{i_{1}} \ldots{ }^{\prime} \theta_{i_{n}}$. Then $\left.{ }^{\prime} \theta_{\boldsymbol{i}} \mid \boldsymbol{i} \in\langle I\rangle_{\alpha}\right\}$ is a basis for ' $\mathbf{f}_{\alpha}$. In particular, each ${ }^{\prime} \mathbf{f}_{\alpha}$ is finite dimensional. Consider the graded dual ' $\mathbf{f}^{*}:=\oplus_{\alpha \in Q_{+}}\left({ }^{\prime} \mathbf{f}_{\alpha}\right)^{*}$. We consider words $\boldsymbol{i} \in\langle I\rangle$ as elements of ' $\mathbf{f}^{*}$, so that $\boldsymbol{i}\left({ }^{\prime} \theta_{\boldsymbol{j}}\right)=\delta_{\boldsymbol{i}, \boldsymbol{j}}$. That is to say, $\left\{\boldsymbol{i} \mid \boldsymbol{i} \in\langle I\rangle_{\alpha}\right\}$ is the basis of ' $\mathbf{f}_{\alpha}^{*}$ dual to the basis $\left\{{ }^{\prime} \theta_{i} \mid \boldsymbol{i} \in\langle I\rangle_{\alpha}\right\}$. 
Let ${ }_{\mathcal{A}}^{\prime} \mathbf{f}$ be the $\mathcal{A}$-subalgebra of 'f generated by $\left\{\left({ }^{\prime} \theta_{i}\right)^{(n)} \mid i \in I, n \in \mathbb{Z}_{\geq 0}\right\}$. This algebra is $Q_{+}$-graded by $\mathcal{A}_{\mathcal{A}}^{\prime} \mathbf{f}=\oplus_{\alpha \in Q_{+}}{ }_{\mathcal{A}}^{\prime} \mathbf{f}_{\alpha}$, where $\mathcal{A}^{\prime} \mathbf{f}_{\alpha}:={ }_{\mathcal{A}}^{\prime} \mathbf{f} \cap^{\prime} \mathbf{f}_{\alpha}$. Given $\boldsymbol{i}=j_{1}^{r_{1}} \ldots j_{m}^{r_{m}} \in\langle I\rangle$ with $j_{n} \neq j_{n+1}$ for $1 \leq n<m$, denote ' $\theta_{(\boldsymbol{i})}:=$ $\left({ }^{\prime} \theta_{j_{1}}\right)^{\left(r_{1}\right)} \ldots\left({ }^{\prime} \theta_{j_{m}}\right)^{\left(r_{m}\right)} \in{ }_{\mathcal{A}}^{\prime} \mathbf{f}$. Then

$$
\left\{{ }^{\prime} \theta_{(i)} \mid \boldsymbol{i} \in\langle I\rangle_{\alpha}\right\}
$$

is an $\mathcal{A}$-basis of ${ }_{\mathcal{A}}^{\prime} \mathbf{f}_{\alpha}$. We also define ${ }_{\mathcal{A}}^{\prime} \mathbf{f}^{*}:=\left\{x \in{ }^{\prime} \mathbf{f}^{*} \mid x\left({ }_{\mathcal{A}}^{\prime} \mathbf{f}\right) \subseteq \mathcal{A}\right\}$, and assign it the induced $Q_{+}$-grading. For every $\alpha \in Q_{+}$, the $\mathcal{A}$-module ${ }_{\mathcal{A}}^{\prime} \mathbf{f}_{\alpha}^{*}$ is free with basis

$$
\left\{[\boldsymbol{i}] ! \boldsymbol{i} \mid \boldsymbol{i} \in\langle I\rangle_{\alpha}\right\}
$$

dual to (2.23).

There is a twisted multiplication on 'f $\otimes$ 'f given by $(x \otimes y)(z \otimes w)=$ $q^{-|y| \cdot|z|} x z \otimes y w$ for homogeneous $x, y, z, w \in{ }^{\prime} \mathbf{f}$. Let $r:{ }^{\prime} \mathbf{f} \rightarrow^{\prime} \mathbf{f} \otimes$ 'f be the algebra homomorphism determined by $r\left({ }^{\prime} \theta_{i}\right)={ }^{\prime} \theta_{i} \otimes 1+1 \otimes^{\prime} \theta_{i}$ for all $i \in I$. By [17, Proposition 1.2.3] there is a unique symmetric bilinear form $(\cdot, \cdot)$ on 'f such that $(1,1)=1$ and

$$
\begin{aligned}
\left({ }^{\prime} \theta_{i},{ }^{\prime} \theta_{j}\right) & =\frac{\delta_{i, j}}{1-q_{i}^{2}} \quad \text { for } i, j \in I, \\
(x y, z) & =(x \otimes y, r(z)), \\
(x, y z) & =(r(x), y \otimes z),
\end{aligned}
$$

where the bilinear form on ' $\mathbf{f} \otimes{ }^{\prime} \mathbf{f}$ is given by $\left(x \otimes x^{\prime}, y \otimes y^{\prime}\right)=(x, y)\left(x^{\prime}, y^{\prime}\right)$.

Define $\mathbf{f}$ to be the quotient of ' $\mathbf{f}$ by the radical of $(\cdot, \cdot)$. Denote the image of ' $\theta_{i}$ in $\mathbf{f}$ by $\theta_{i}$. The $Q_{+}$-grading on 'f descends to a $Q_{+}$-grading on $\mathbf{f}$ with $\left|\theta_{i}\right|=i$. Let ${ }_{\mathcal{A}} \mathbf{f}$ be the $\mathcal{A}$-subalgebra of $\mathbf{f}$ generated by $\theta_{i}^{(n)}$ for $i \in I, n \in \mathbb{Z}_{\geq 0}$. This algebra is $Q_{+}$-graded by ${ }_{\mathcal{A}} \mathbf{f}_{\alpha}:={ }_{\mathcal{A}} \mathbf{f} \cap \mathbf{f}_{\alpha}$. Given $\boldsymbol{i}=j_{1}^{r_{1}} \ldots j_{m}^{r_{m}} \in\langle I\rangle$ with $j_{n} \neq j_{n+1}$ for $1 \leq n<m$, denote $\theta_{i}:=\theta_{j_{1}}^{r_{1}} \ldots \theta_{j_{m}}^{r_{m}}$ and

$$
\theta_{(\boldsymbol{i})}:=\theta_{j_{1}}^{\left(r_{1}\right)} \ldots \theta_{j_{m}}^{\left(r_{m}\right)} \in{ }_{\mathcal{A}} \mathbf{f} .
$$

We recall the definition of the $P B W$ basis of ${ }_{\mathcal{A}} \mathbf{f}$ from [17, Part VI]. Recall that a reduced decomposition $w_{0}=s_{i_{1}} \ldots s_{i_{N}}$ yields a total order on the positive roots $\Phi_{+}=\left\{\beta_{1}>\cdots>\beta_{N}\right\}$, with $\beta_{N+1-k}=s_{i_{1}} \ldots s_{i_{k-1}}\left(\alpha_{i_{k}}\right)$. Now, embed ${ }_{\mathcal{A}} \mathbf{f}$ into the upper half of the full quantum group via $\theta_{i} \mapsto E_{i}$ and take the braid group generators $T_{i}:=T_{i,+}^{\prime \prime}$ from [17, 37.1.3]. For $1 \leq k \leq N$, we define

$$
E_{\beta_{N+1-k}}:=T_{i_{1}} \ldots T_{i_{k-1}}\left(\theta_{i_{k}}\right) \in{ }_{\mathcal{A}} \mathbf{f}_{\beta_{N+1-k}} .
$$

For a sequence $\pi=\left(p_{1}, \ldots p_{N}\right) \in \mathbb{Z}_{\geq 0}^{N}$, we set

$$
E_{\pi}:=E_{\beta_{1}}^{\left(p_{1}\right)} \ldots E_{\beta_{N}}^{\left(p_{N}\right)}
$$

and also define

$$
l_{\pi}:=\prod_{r=1}^{N} \prod_{s=1}^{p_{k}} \frac{1}{1-q_{\beta_{r}}^{2 s}} .
$$

The next theorem now gives a PBW basis of ${ }_{\mathcal{A}} \mathbf{f}_{\alpha}$. 
Theorem 2.8. The set $\left\{E_{\pi} \mid \pi \in \Pi(\alpha)\right\}$ is an $\mathcal{A}$-basis of ${ }_{\mathcal{A}} \mathbf{f}_{\alpha}$. Furthermore:

$$
\left(E_{\pi}, E_{\sigma}\right)=\delta_{\pi, \sigma} l_{\pi}
$$

Proof. This follows from Corollary 41.1.4(b), Propositions 41.1.7, 38.2.3, and Lemma 1.4.4 of Lusztig [17].

Consider the graded dual $\mathbf{f}^{*}:=\oplus_{\alpha \in Q_{+}} \mathbf{f}_{\alpha}^{*}$. The map $r^{*}: \mathbf{f}^{*} \otimes \mathbf{f}^{*} \rightarrow \mathbf{f}^{*}$ gives $\mathbf{f}^{*}$ the structure of an associative algebra. Let

$$
\kappa: \mathbf{f}^{*} \hookrightarrow{ }^{\prime} \mathbf{f}^{*}
$$

be the map dual to the quotient map $\xi:{ }^{\prime} \mathbf{f} \rightarrow \mathbf{f}$. Set ${ }_{\mathcal{A}} \mathbf{f}^{*}:=\left\{x \in \mathbf{f}^{*} \mid\right.$ $\left.x\left({ }_{\mathcal{A}} \mathbf{f}\right) \subseteq \mathcal{A}\right\}$ with the induced $Q_{+}$-grading. Given $i \in I$, we denote by $\theta_{i}^{*}: \mathbf{f}^{*} \rightarrow \mathbf{f}^{*}$ the dual map to the map $\mathbf{f} \rightarrow \mathbf{f}, x \mapsto x \theta_{i}$. Then the divided power $\left(\theta_{i}^{*}\right)^{(n)}: \mathbf{f}^{*} \rightarrow \mathbf{f}^{*}$ is dual to the map $x \mapsto x \theta_{i}^{(n)}$. Clearly $\left(\theta_{i}^{*}\right)^{(n)}$

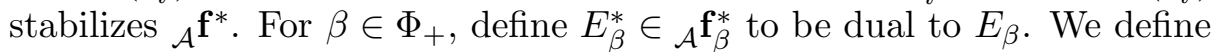

$$
\left(E_{\beta}^{*}\right)^{\langle m\rangle}:=q_{\beta}^{m(m-1) / 2}\left(E_{\beta}^{*}\right)^{m} \text { and } E_{\pi}^{*}:=\left(E_{\beta_{1}}^{*}\right)^{\left\langle p_{1}\right\rangle} \ldots\left(E_{\beta_{N}}^{*}\right)^{\left\langle p_{N}\right\rangle}
$$

for $m \geq 0$, and any sequence $\pi=\left(p_{1}, \ldots p_{N}\right) \in \mathbb{Z}_{\geq 0}^{N}$. The next well-known result gives the dual $P B W$ basis of ${ }_{\mathcal{A}} \mathbf{f}^{*}$.

Theorem 2.9. The set $\left\{E_{\pi}^{*} \mid \pi \in \Pi(\alpha)\right\}$ is the $\mathcal{A}$-basis of ${ }_{\mathcal{A}} \mathbf{f}_{\alpha}^{*}$ dual to the $P B W$ basis of Theorem 2.8.

Proof. It easily follows from the properties of the Lusztig bilinear form, the definition of the product on $\mathbf{f}^{*}$ and Theorem 2.8 that the linear functions $\left(E_{\pi},-\right)$ and $l_{\pi} E_{\pi}^{*}$ on $\mathbf{f}$ are equal. It remains to apply Theorem 2.8 one more time.

Example 2.10. Let $C=A_{2}$, and $w_{0}=s_{1} s_{2} s_{1}$. Then $E_{\alpha_{1}+\alpha_{2}}=T_{1,+}^{\prime \prime}\left(E_{2}\right)=$ $E_{1} E_{2}-q E_{2} E_{1}$, and, switching back to $\theta$ 's, the PBW basis of $\mathcal{A}_{\alpha_{1}+\alpha_{2}}$ is $\left\{\theta_{2} \theta_{1}, \theta_{1} \theta_{2}-q \theta_{2} \theta_{1}\right\}$. Using the defining properties of Lusztig's bilinear form, one can easily check that $\left(E_{\alpha_{2}} E_{\alpha_{1}}, E_{\alpha_{2}} E_{\alpha_{1}}\right)=\frac{1}{\left(1-q^{2}\right)^{2}},\left(E_{\alpha_{1}+\alpha_{2}}, E_{\alpha_{1}+\alpha_{2}}\right)=$ $\frac{1}{\left(1-q^{2}\right)}$, and $\left(E_{\alpha_{2}} E_{\alpha_{1}}, E_{\alpha_{1}+\alpha_{2}}\right)=0$. Finally the dual basis is $\{(12),(21)+$ $q(12)\}$.

2.7. Categorification of ${ }_{\mathcal{A}} \mathbf{f}$ and ${ }_{\mathcal{A}} \mathbf{f}^{*}$. Now we state the fundamental categorification theorem proved in $\left[7,8\right.$, see also [20]. We denote by $\left[R_{0}\right]$ the class of the left regular representation of the trivial algebra $R_{0} \cong F$.

Theorem 2.11. There is a unique $\mathcal{A}$-linear isomorphism $\gamma:{ }_{\mathcal{A}} \mathbf{f} \stackrel{\sim}{\rightarrow}[R$-proj] such that $1 \mapsto\left[R_{0}\right]$ and $\gamma\left(x \theta_{i}^{(n)}\right)=\theta_{i}^{(n)}(\gamma(x))$ for all $x \in{ }_{\mathcal{A}} \mathbf{f}, i \in I$, and $n \geq 1$. Under this isomorphism:

(1) $\gamma\left({ }_{\mathcal{A}} \mathbf{f}_{\alpha}\right)=\left[R_{\alpha}\right.$-proj];

(2) the multiplication ${ }_{\mathcal{A}} \mathbf{f}_{\alpha} \otimes{ }_{\mathcal{A}} \mathbf{f}_{\beta} \rightarrow{ }_{\mathcal{A}} \mathbf{f}_{\alpha+\beta}$ corresponds to the product on $\left[R\right.$-proj] induced by the exact functor $\operatorname{Ind}_{\alpha, \beta}$;

(3) for $\boldsymbol{i} \in\langle I\rangle_{\alpha}$ we have $\gamma\left(\theta_{\boldsymbol{i}}\right)=\left[R_{\alpha} e(\boldsymbol{i})\right]$;

(4) for $x, y \in{ }_{\mathcal{A}} \mathbf{f}$ we have $(x, y)=(\gamma(x), \gamma(y))$. 
Let $M$ be a finite dimensional graded $R_{\alpha}$-module. Define the $q$-character of $M$ as follows:

$$
\operatorname{ch}_{q} M:=\sum_{i \in\langle I\rangle_{\alpha}}\left(\operatorname{dim}_{q} M_{i}\right) \boldsymbol{i} \in{ }_{\mathcal{A}}^{\prime} \mathbf{f}^{*} .
$$

The $q$-character map $\operatorname{ch}_{q}: R_{\alpha}$-mod $\rightarrow{ }_{\mathcal{A}}^{\prime} \mathbf{f}^{*}$ factors through to give an $\mathcal{A}$ linear map from the Grothendieck group

$$
\operatorname{ch}_{q}:\left[R_{\alpha}-\bmod \right] \rightarrow{ }_{\mathcal{A}}^{\prime} \mathbf{f}^{*} .
$$

We now state a dual result to Theorem 2.11, see [11, Theorem 4.4].

Theorem 2.12. There is a unique $\mathcal{A}$-linear isomorphism $\gamma^{*}:[R$-mod $] \stackrel{\sim}{\rightarrow}$ ${ }_{\mathcal{A}} \mathbf{f}^{*}$ with the following properties:

(1) $\gamma^{*}\left(\left[R_{0}\right]\right)=1$;

(2) $\gamma^{*}\left(\left(\theta_{i}^{*}\right)^{(n)}(x)\right)=\left(\theta_{i}^{*}\right)^{(n)}\left(\gamma^{*}(x)\right)$ for all $x \in[R$-mod], $i \in I, n \geq 1$;

(3) the following triangle is commutative:

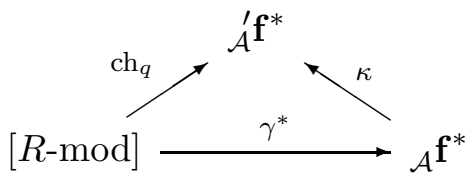

(4) $\gamma^{*}\left(\left[R_{\alpha}\right.\right.$-mod $\left.]\right)={ }_{\mathcal{A}} \mathbf{f}_{\alpha}^{*}$ for all $\gamma \in Q_{+}$;

(5) under the isomorphism $\gamma^{*}$, the multiplication ${ }_{\mathcal{A}} \mathbf{f}_{\alpha}^{*} \otimes{ }_{\mathcal{A}} \mathbf{f}_{\beta}^{*} \rightarrow{ }_{\mathcal{A}} \mathbf{f}_{\alpha+\beta}^{*}$ corresponds to the product on $\left[R\right.$-mod] induced by $\operatorname{Ind}_{\alpha, \beta}$;

We conclude with McNamara's result on the categorification of the dual PBW-basis (see also [6] for simply laced Lie types):

Lemma 2.13. For every $\pi \in \Pi(\alpha)$ we have $\gamma^{*}([\bar{\Delta}(\pi)])=E_{\pi}^{*}$.

Proof. By [19, Theorem 3.1(1)], we have $\gamma^{*}([L(\beta)])=E_{\beta}^{*}$ for all $\beta \in \Phi_{+}$. The general case then follows from Theorem $2.12(5)$ and the definition (2.22) of $\bar{\Delta}(\pi)$.

2.8. A dimension formula. In this section we obtain a dimension formula for $R_{\alpha}$, which can be viewed as a combinatorial shadow of the affine quasihereditary structure on it. The idea of the proof comes from [1, Theorem 4.20]. An independent but much less elementary proof can be found in [2, Corollary 3.15].

Recall the element $\theta_{(\boldsymbol{i})} \in{ }_{\mathcal{A}} \mathbf{f}$ from (2.25) and the scalar $[\boldsymbol{i}] ! \in \mathcal{A}$ from (2.1). We note that Lemma 2.14 and Theorem 2.15 do not require the assumption that the Cartan matrix $A$ is of finite type, adopted elsewhere in the paper.

Lemma 2.14. Let $V^{1}, \ldots, V^{m} \in R_{\alpha}$-mod, and let $v^{n}:=\gamma^{*}\left(\left[V^{n}\right]\right) \in{ }_{\mathcal{A}} \mathbf{f}_{\alpha}^{*}$ for $n=1, \ldots, m$. Assume that $\left\{v^{1}, \ldots, v^{m}\right\}$ is an $\mathcal{A}$-basis of $\mathcal{A}_{\alpha}^{*}$. Let $\left\{v_{1}, \ldots, v_{m}\right\}$ be the dual basis of ${ }_{\mathcal{A}} \mathbf{f}_{\alpha}$. Then for every $\boldsymbol{i} \in\langle I\rangle_{\alpha}$, we have

$$
\theta_{(\boldsymbol{i})}=\sum_{n=1}^{m} \frac{\operatorname{dim}_{q} V_{\boldsymbol{i}}^{n}}{[\boldsymbol{i}] !} v_{n} .
$$


Proof. Recall the map $\kappa$ from (2.27) dual to the natural projection $\xi$ : 'f $\rightarrow \mathbf{f}$. By Theorem 2.12 we have for any $1 \leq n \leq m$ :

$$
\kappa\left(v^{n}\right)=\kappa\left(\gamma^{*}\left(\left[V^{n}\right]\right)\right)=\operatorname{ch}_{q}\left(\left[V^{n}\right]\right)=\sum_{\boldsymbol{i} \in\langle I\rangle_{\alpha}}\left(\operatorname{dim}_{q} V_{\boldsymbol{i}}^{n}\right) \boldsymbol{i}=\sum_{\boldsymbol{i} \in\langle I\rangle_{\alpha}} \frac{\operatorname{dim}_{q} V_{\boldsymbol{i}}^{n}}{[\boldsymbol{i}] !}[\boldsymbol{i}] ! \boldsymbol{i} .
$$

Recalling (2.23) and (2.24), $\left\{^{\prime} \theta_{(i)} \mid \boldsymbol{i} \in\langle I\rangle_{\alpha}\right\}$ and $\left\{[\boldsymbol{i}] ! \boldsymbol{i} \mid \boldsymbol{i} \in\langle I\rangle_{\alpha}\right\}$ is a pair of dual bases in ${ }_{\mathcal{A}}^{\prime} \mathbf{f}_{\alpha}$ and ${ }_{\mathcal{A}}^{\prime} \mathbf{f}_{\alpha}^{*}$. So, using our expression for $\kappa\left(v^{n}\right)$, we can now get by dualizing:

$$
\begin{aligned}
\theta_{(\boldsymbol{i})} & =\xi\left({ }^{\prime} \theta_{(\boldsymbol{i})}\right)=\sum_{n=1}^{m} v^{n}\left(\xi\left({ }^{\prime} \theta_{(\boldsymbol{i})}\right)\right) v_{n} \\
& =\sum_{n=1}^{m} \kappa\left(v^{n}\right)\left({ }^{\prime} \theta_{(\boldsymbol{i})}\right) v_{n}=\sum_{n=1}^{m} \sum_{\boldsymbol{j} \in\langle I\rangle_{\alpha}} \frac{\operatorname{dim}_{q} V_{\boldsymbol{j}}^{n}}{[\boldsymbol{j}] !}[\boldsymbol{j}] ! \boldsymbol{j}\left({ }^{\prime} \theta_{(\boldsymbol{i})}\right) v_{n} \\
& =\sum_{n=1}^{m} \sum_{\boldsymbol{j} \in\langle I\rangle_{\alpha}} \frac{\operatorname{dim}_{q} V_{\boldsymbol{j}}^{n}}{[\boldsymbol{j}] !} \delta_{\boldsymbol{i}, \boldsymbol{j}} v_{n}=\sum_{n=1}^{m} \frac{\operatorname{dim}_{q} V_{\boldsymbol{i}}^{n}}{[\boldsymbol{i}] !} v_{n},
\end{aligned}
$$

as required.

Theorem 2.15. With the assumptions of Lemma 2.14, for every $\boldsymbol{i}, \boldsymbol{j} \in\langle I\rangle_{\alpha}$, we have

$$
\operatorname{dim}_{q}\left(e(\boldsymbol{i}) R_{\alpha} e(\boldsymbol{j})\right)=\sum_{n, k=1}^{m}\left(\operatorname{dim}_{q} V_{\boldsymbol{i}}^{n}\right)\left(\operatorname{dim}_{q} V_{\boldsymbol{j}}^{k}\right)\left(v_{n}, v_{k}\right)
$$

In particular,

$$
\operatorname{dim}_{q}\left(R_{\alpha}\right)=\sum_{n, k=1}^{m}\left(\operatorname{dim}_{q} V^{n}\right)\left(\operatorname{dim}_{q} V^{k}\right)\left(v_{n}, v_{k}\right)
$$

Proof. Theorem $2.11(3)$ shows that $\left[R_{\alpha} e(\boldsymbol{i})\right]=\gamma\left(\theta_{\boldsymbol{i}}\right)=\gamma\left([\boldsymbol{i}] ! \theta_{(\boldsymbol{i})}\right)$. Using the definitions and Theorem 2.11(4), we have

$$
\begin{aligned}
\operatorname{dim}_{q}\left(e(\boldsymbol{i}) R_{\alpha} e(\boldsymbol{j})\right) & =\operatorname{dim}_{q}\left(\left(R_{\alpha} e(\boldsymbol{i})\right)^{\tau} \otimes_{R_{\alpha}} R_{\alpha} e(\boldsymbol{j})\right) \\
& =\left(\left[R_{\alpha} e(\boldsymbol{i})\right],\left[R_{\alpha} e(\boldsymbol{j})\right]\right)=\left([\boldsymbol{i}] ! \theta_{(\boldsymbol{i})},[\boldsymbol{j}] ! \theta_{(\boldsymbol{j})}\right) .
\end{aligned}
$$

Now, by Lemma 2.14 we see that

$$
\left([\boldsymbol{i}] ! \theta_{(\boldsymbol{i})},[\boldsymbol{j}] ! \theta_{(\boldsymbol{j})}\right)=\left(\sum_{n=1}^{m}\left(\operatorname{dim}_{q} V_{\boldsymbol{i}}^{n}\right) v_{n}, \sum_{k=1}^{n}\left(\operatorname{dim}_{q} V_{\boldsymbol{j}}^{k}\right) v_{k}\right),
$$

which implies the theorem.

Recall the scalar $l_{\pi}$ from (2.26), the module $\bar{\Delta}(\pi)$ from (2.22), and PBWbasis elements $E_{\pi}$ from 2.6 . 
Corollary 2.16. For every $\boldsymbol{i}, \boldsymbol{j} \in\langle I\rangle_{\alpha}$, we have

$$
\operatorname{dim}_{q}\left(e(\boldsymbol{i}) R_{\alpha} e(\boldsymbol{j})\right)=\sum_{\pi \in \Pi(\alpha)}\left(\operatorname{dim}_{q} \bar{\Delta}(\pi)_{\boldsymbol{i}}\right)\left(\operatorname{dim}_{q} \bar{\Delta}(\pi)_{\boldsymbol{j}}\right) l_{\pi} .
$$

In particular,

$$
\operatorname{dim}_{q}\left(R_{\alpha}\right)=\sum_{\pi \in \Pi(\alpha)}\left(\operatorname{dim}_{q} \bar{\Delta}(\pi)\right)^{2} l_{\pi} .
$$

Proof. By Lemma 2.13, we have $\gamma^{*}(\bar{\Delta}(\pi))=E_{\pi}^{*}$ for all $\pi \in \Pi(\alpha)$. Moreover, by Theorem 2.9, $\left\{E_{\pi}^{*} \mid \pi \in \Pi(\alpha)\right\}$ and $\left\{E_{\pi} \mid \pi \in \Pi(\alpha)\right\}$ is a pair of dual bases in ${ }_{\mathcal{A}} \mathbf{f}_{\alpha}^{*}$ and ${ }_{\mathcal{A}} \mathbf{f}_{\alpha}$. Finally, $\left(E_{\pi}, E_{\sigma}\right)=\delta_{\pi, \sigma} l_{\pi}$ by Theorem [2.8, It remains to apply Theorem 2.15.

\section{Affine cellular structure}

Throughout this section we fix $\alpha \in Q_{+}$and a total order $\leq$on the set $\Pi(\alpha)$ of root partitions of $\alpha$, which refines the bilexicographic partial order (2.21).

3.1. Some special word idempotents. Recall from Section 2.5 that for each $\beta \in \Phi_{+}$, we have a cuspidal module $L(\beta)$. Every irreducible $R_{\alpha}$-module $L$ has a word space $L_{\boldsymbol{i}}$ such that the lowest degree component of $L_{\boldsymbol{i}}$ is onedimensional, see for example [9, Lemma 2.30] or [2, Lemma 4.5] for two natural choices. From now on, for each $\beta \in \Phi_{+}$we make an arbitrary choice of such word $\boldsymbol{i}_{\beta}$ for the cuspidal module $L(\beta)$.

For $\pi=\left(\beta_{1}^{p_{1}}, \ldots, \beta_{N}^{p_{N}}\right) \in \Pi(\alpha)$, define

$$
\begin{aligned}
\boldsymbol{i}_{\pi} & :=\boldsymbol{i}_{\beta_{1}}^{p_{1}} \ldots \boldsymbol{i}_{\beta_{N}}^{p_{N}}, \\
I_{\pi} & :=\sum_{\sigma \geq \pi} R_{\alpha} e\left(\boldsymbol{i}_{\sigma}\right) R_{\alpha}, \\
I_{>\pi} & :=\sum_{\sigma>\pi} R_{\alpha} e\left(\boldsymbol{i}_{\sigma}\right) R_{\alpha},
\end{aligned}
$$

the sums being over $\sigma \in \Pi(\alpha)$. We also consider the (non-unital) embedding of algebras:

$$
\iota_{\pi}:=\iota_{p_{1} \beta_{1}, \ldots, p_{N} \beta_{N}}: R_{p_{1} \beta_{1}} \otimes \cdots \otimes R_{p_{N} \beta_{N}} \hookrightarrow R_{\alpha},
$$

whose image is the parabolic subalgebra

$$
R_{\pi}:=R_{p_{1} \beta_{1}, \ldots, p_{N} \beta_{N}} .
$$

Lemma 3.1. If a two-sided ideal $J$ of $R_{\alpha}$ contains all idempotents $e\left(\boldsymbol{i}_{\pi}\right)$ with $\pi \in \Pi(\alpha)$, then $J=R_{\alpha}$.

Proof. If $J \neq R_{\alpha}$, let $I$ be a maximal left ideal containing $J$. Then $R_{\alpha} / I \cong L(\pi)$ for some $\pi$. Then $e\left(i_{\pi}\right) L(\pi) \neq 0$, which contradicts the assumption that $e\left(\boldsymbol{i}_{\pi}\right) \in J$. This argument proves the lemma over any field, and then it also follows for $\mathbb{Z}$. 
Lemma 3.2. Let $\pi \in \Pi(\alpha)$ and $e \in R_{\alpha}$ a homogeneous idempotent. If $e L(\sigma)=0$ for all $\sigma \leq \pi$, then $e \in I_{>\pi}$.

Proof. Let $I$ be any maximal (graded) left ideal containing $I_{>\pi}$. Then $R_{\alpha} / I \cong L(\sigma)$ for some $\sigma \in \Pi(\alpha)$ such that $\sigma \leq \pi$. Indeed, if we had $\sigma>\pi$ then by definition $e\left(\boldsymbol{i}_{\sigma}\right) \in I_{>\pi} \subseteq I$, and so $e\left(\boldsymbol{i}_{\sigma}\right) L(\sigma)=e\left(\boldsymbol{i}_{\sigma}\right)\left(R_{\alpha} / I\right)=0$, which is a contradiction.

We have shown that $e$ is contained in every maximal left ideal containing $I_{>\pi}$. By a standard argument, explained in [10, Lemma 5.8], we conclude that $e \in I_{>\pi}$.

Corollary 3.3. Suppose that $\alpha=p \beta$ for some $p \geq 1$ and $\beta \in \Phi_{+}$. Let $\boldsymbol{i} \in\langle I\rangle_{\alpha}$. If e $(\boldsymbol{i}) L\left(\beta^{p}\right)=0$, then $e(\boldsymbol{i}) \in I_{>\left(\beta^{p}\right)}$.

Proof. This follows from Lemma 2.5 together with Proposition 3.2 ,

Lemma 3.4. Let $\pi=\left(\beta_{1}^{p_{1}} \ldots \beta_{N}^{p_{N}}\right) \in \Pi(\alpha)$. Then $R_{\pi} \subseteq I_{\pi}$.

Proof. By Lemma 3.1, we have

$$
R_{p_{n} \beta_{n}}=\sum_{\pi^{(n)} \in \Pi\left(p_{n} \beta_{n}\right)} R_{p_{n} \beta_{n}} e\left(\boldsymbol{i}_{\pi^{(n)}}\right) R_{p_{n} \beta_{n}}
$$

for all $n=1, \ldots, N$. Therefore the image of $\iota_{\pi}$ equals

$$
\sum R_{\pi} e\left(\boldsymbol{i}_{\pi^{(1)}} \ldots \boldsymbol{i}_{\pi^{(N)}}\right) R_{\pi}
$$

where the sum is over all $\pi^{(1)} \in \Pi\left(p_{1} \beta_{1}\right), \ldots, \pi^{(N)} \in \Pi\left(p_{N} \beta_{N}\right)$. Fix $\pi^{(n)} \in$ $\Pi\left(p_{n} \beta_{n}\right)$ for all $n=1, \ldots, N$. If $\pi^{(n)}=\left(\beta_{n}^{p_{n}}\right)$ for every $n$, then $\boldsymbol{i}_{\pi^{(1)}} \ldots \boldsymbol{i}_{\pi^{(N)}}=$ $\boldsymbol{i}_{\pi}$, and the corresponding term of (3.1) is in $I_{\pi}$ by definition.

Let us now assume that $\pi^{(k)} \neq\left(\beta_{k}^{p_{k}}\right)$ for some $k$. In view of Lemma 2.5. we have $\pi^{(k)}>\left(\beta_{k}^{p_{k}}\right)$. For any $\sigma \in \Pi(\alpha)$ we have $e\left(\boldsymbol{i}_{\pi^{(1)}} \ldots \boldsymbol{i}_{\pi^{(N)}}\right) \bar{\Delta}(\sigma) \subseteq$ $\operatorname{Res}_{\pi} \bar{\Delta}(\sigma)$, and by Theorem 2.6(vi), if $\sigma<\pi$ then $\operatorname{Res}_{\pi} \bar{\Delta}(\sigma)=0$. Furthermore, for $\sigma=\pi$ we have, by Theorem 2.6(vi) applied again

$$
\begin{aligned}
e\left(\boldsymbol{i}_{\pi^{(1)}} \ldots \boldsymbol{i}_{\pi^{(N)}}\right) \bar{\Delta}(\pi) & =e\left(\boldsymbol{i}_{\pi^{(1)}} \ldots \boldsymbol{i}_{\pi^{(N)}}\right) \operatorname{Res}_{\pi} \bar{\Delta}(\pi) \\
& =e\left(\boldsymbol{i}_{\pi^{(1)}} \ldots \boldsymbol{i}_{\pi^{(N)}}\right)\left(\bar{\Delta}\left(\beta_{1}^{p_{1}}\right) \otimes \cdots \otimes \bar{\Delta}\left(\beta_{N}^{p_{N}}\right)\right) \\
& \subseteq\left(\operatorname{Res}_{\pi^{(1)}} \bar{\Delta}\left(\beta_{1}^{p_{1}}\right)\right) \otimes \cdots \otimes\left(\operatorname{Res}_{\pi^{(N)}} \bar{\Delta}\left(\beta_{N}^{p_{N}}\right)\right),
\end{aligned}
$$

which is zero since $\operatorname{Res}_{\pi^{(k)}} \bar{\Delta}\left(\beta_{k}^{p_{k}}\right)=0$ by Theorem 2.6)(vi) again. We have shown that for all $\sigma \leq \pi$ we have $e\left(\boldsymbol{i}_{\pi^{(1)}} \ldots \boldsymbol{i}_{\pi^{(N)}}\right) \bar{\Delta}(\sigma)=0$, and consequently $e\left(\boldsymbol{i}_{\pi^{(1)}} \ldots \boldsymbol{i}_{\pi^{(N)}}\right) L(\sigma)=0$. Applying Lemma 3.2, we have that $e\left(\boldsymbol{i}_{\pi^{(1)}} \ldots \boldsymbol{i}_{\pi^{(N)}}\right) \in I_{>\pi} \subseteq I_{\pi}$.

The following result will often allow us to reduce to the case of a smaller height.

Proposition 3.5. Let $\gamma_{1}, \ldots, \gamma_{m} \in Q_{+}, 1 \leq k \leq m$, and $\pi_{0} \in \Pi\left(\gamma_{k}\right)$. Assume that $\pi \in \Pi\left(\gamma_{1}+\cdots+\gamma_{m}\right)$ is such that all idempotents from the set

$$
E=\left\{e\left(\boldsymbol{i}_{\pi^{(1)}} \ldots \boldsymbol{i}_{\pi^{(m)}}\right) \mid \pi^{(n)} \in \Pi\left(\gamma_{n}\right) \text { for all } n=1, \ldots, m \text { and } \pi^{(k)}>\pi_{0}\right\}
$$


annihilate the irreducible modules $L(\sigma)$ for all $\sigma \leq \pi$. Then

$$
\iota_{\gamma_{1}, \ldots, \gamma_{m}}\left(R_{\gamma_{1}} \otimes \cdots \otimes R_{\gamma_{k-1}} \otimes I_{>\pi_{0}} \otimes R_{\gamma_{k+1}} \otimes \cdots \otimes R_{\gamma_{m}}\right) \subseteq I_{>\pi} .
$$

Proof. We may assume that $\gamma_{k} \neq 0$ since otherwise $I_{>\pi_{0}}=0$, and the result is clear. By Lemma 3.1, we have $R_{\gamma_{n}}=\sum_{\pi^{(n)} \in \Pi\left(\gamma_{n}\right)} R_{\gamma_{n}} e\left(\boldsymbol{i}_{\pi^{(n)}}\right) R_{\gamma_{n}}$ for all $n=1, \ldots, m$, and by definition, we have $I_{>\pi_{0}}=\sum_{\pi^{(k)}>\pi_{0}} R_{\gamma_{k}} e\left(\boldsymbol{i}_{\pi^{(k)}}\right) R_{\gamma_{k}}$. Therefore the left hand side of (3.2) equals $\sum_{e \in E} R_{\gamma_{1}, \ldots, \gamma_{m}} e R_{\gamma_{1}, \ldots, \gamma_{m}}$. The result now follows by applying Lemma 3.2 .

Recall from Lemma 3.4 that $\operatorname{im}\left(\iota_{\pi}\right) \subseteq I_{\pi}$.

Corollary 3.6. Let $\pi=\left(\beta_{1}^{p_{1}}, \ldots, \beta_{N}^{p_{N}}\right) \in \Pi(\alpha)$ and $1 \leq k \leq N$. Then

$$
\iota_{\pi}\left(R_{p_{1} \beta_{1}} \otimes \cdots \otimes R_{p_{k-1} \beta_{k-1}} \otimes I_{>\left(\beta_{k}^{p_{k}}\right)} \otimes R_{p_{k+1} \beta_{k+1}} \otimes \cdots \otimes R_{p_{N} \beta_{N}}\right) \subseteq I_{>\pi} .
$$

In particular, the composite map $R_{\pi} \stackrel{\iota_{\pi}}{\longrightarrow} I_{\pi} \longrightarrow I_{\pi} / I_{>\pi}$ factors through the quotient $R_{p_{1} \beta_{1}} / I_{>\left(\beta_{1}^{p_{1}}\right)} \otimes \cdots \otimes R_{p_{N} \beta_{N}} / I_{>\left(\beta_{N}^{p_{N}}\right)}$.

Proof. Apply Proposition 3.5 with $m=N, \gamma_{n}=p_{n} \beta_{n}$, for $1 \leq n \leq N$, $\pi_{0}=\left(\beta_{k}^{p_{k}}\right)$, and $\pi=\pi$. We have to prove that any $e=e\left(\boldsymbol{i}_{\pi^{(1)}} \ldots \boldsymbol{i}_{\pi^{(N)}}\right) \in E$ annihilates all $L(\sigma)$ for $\sigma \leq \pi$. We prove more, namely that $e$ annihilates $\bar{\Delta}(\sigma)$ for all $\sigma \leq \pi$. By Theorem 2.6(vi):

$$
\begin{aligned}
e \bar{\Delta}(\sigma) & =e \operatorname{Res}_{\pi} \bar{\Delta}(\sigma)=e \delta_{\pi, \sigma}\left(L\left(\beta_{1}\right)^{\circ p_{1}} \otimes \cdots \otimes L\left(\beta_{1}\right)^{\circ p_{1}}\right) \\
& =\delta_{\pi, \sigma} e\left(\boldsymbol{i}_{\pi^{(1)}}\right) L\left(\beta_{1}\right)^{o p_{1}} \nabla \cdots \otimes e\left(\boldsymbol{i}_{\pi^{(N)}}\right) L\left(\beta_{N}\right)^{\circ p_{N}},
\end{aligned}
$$

which is zero since

$$
e\left(\boldsymbol{i}_{\pi^{(k)}}\right) L\left(\beta_{k}\right)^{\circ p_{k}}=e\left(\boldsymbol{i}_{\pi^{(k)}}\right) \operatorname{Res}_{\pi^{(k)}} L\left(\beta_{k}\right)^{\circ p_{k}}=0
$$

by Theorem 2.6(vi) again.

Corollary 3.7. For $\beta \in \Phi_{+}$and $a, b, c \in \mathbb{Z}_{\geq 0}$ we have

$$
\iota_{a \beta, b \beta, c \beta}\left(R_{a \beta} \otimes I_{>\left(\beta^{b}\right)} \otimes R_{c \beta}\right) \subseteq I_{>\left(\beta^{a+b+c}\right)} .
$$

Proof. We apply Proposition 3.5 with $m=3, k=2, \gamma_{1}=a \beta, \gamma_{2}=$ $b \beta, \gamma_{3}=c \beta, \pi_{0}=\left(\beta^{b}\right)$ and $\pi=\left(\beta^{a+b+c}\right)$. Pick an idempotent $e=$ $e\left(\boldsymbol{i}_{\pi^{(1)}} \boldsymbol{i}_{\pi^{(2)}} \boldsymbol{i}_{\pi^{(3)}}\right) \in E$. Since $\pi$ is the minimal element of $\Pi((a+b+c) \beta)$, it suffices to prove that $e L(\pi)=0$. Note that $e L(\pi)=e \operatorname{Res}_{a \beta, b \beta, c \beta} L(\pi)$, so using Lemma 2.4, we just need to show that $e\left(L(\beta)^{\circ a} \otimes L(\beta)^{\circ b} \otimes L(\beta)^{\circ c}\right)=0$. But

$e\left(L(\beta)^{\circ a} \otimes L(\beta)^{\circ b} \otimes L(\beta)^{\circ c}\right)=e\left(\boldsymbol{i}_{\pi^{(1)}}\right) L(\beta)^{\circ a} \otimes e\left(\boldsymbol{i}_{\pi^{(2)}}\right) L(\beta)^{\circ b} \otimes e\left(\boldsymbol{i}_{\pi^{(3)}}\right) L(\beta)^{\circ c}$ is zero, since $e\left(\boldsymbol{i}_{\pi^{(2)}}\right) L(\beta)^{\circ b}=e\left(\boldsymbol{i}_{\pi^{(2)}}\right) \operatorname{Res}_{\pi^{(2)}} L(\beta)^{\circ b}=0$ by Theorem [2.6(vi).

Repeated application of Corollary 3.7 gives the following result.

Corollary 3.8. For $\beta \in \Phi_{+}$and $p \in \mathbb{Z}_{>0}$ we have

$$
\iota_{\beta, \ldots, \beta}\left(R_{\beta} \otimes \cdots \otimes I_{>(\beta)} \otimes \cdots \otimes R_{\beta}\right) \subseteq I_{>\left(\beta^{p}\right)} .
$$


3.2. Basic notation concerning cellular bases. Let $\beta$ be a fixed positive root of height $d$. Recall that we have made a choice of $\boldsymbol{i}_{\beta}$ so that in the word space $e\left(\boldsymbol{i}_{\beta}\right) L(\beta)$ of the cuspidal module, the lowest degree part is 1dimensional. We fix its spanning vector $v_{\beta}^{-}$defined over $\mathbb{Z}$, see Lemma 2.7 . Similarly, the highest degree part is spanned over $\mathbb{Z}$ by some $v_{\beta}^{+}$.

We consider the element of the symmetric group $w_{\beta, r} \in \mathfrak{S}_{p d}$

$$
w_{\beta, r}:=\prod_{k=1}^{d}((r-1) d+k, r d+k) .
$$

which permutes the $r$ th and the $(r+1)$ st ' $d$-blocks'. Now define

$$
\psi_{\beta, r}:=\psi_{w_{\beta, r}} \in R_{p \beta} \text {. }
$$

Moreover, for $u \in \mathfrak{S}_{p}$ with a fixed reduced decomposition $u=s_{r_{1}} \ldots s_{r_{m}}$, define the elements

$$
\begin{aligned}
& w_{\beta, u}:=w_{\beta, r_{1}} \ldots w_{\beta, r_{m}} \in \mathfrak{S}_{p d}, \\
& \psi_{\beta, u}:=\psi_{\beta, r_{1}} \ldots \psi_{\beta, r_{m}} \in R_{p \beta} .
\end{aligned}
$$

In Section 5, we will explicitly define homogeneous elements

$$
\delta_{\beta}, D_{\beta}, y_{\beta} \in e\left(\boldsymbol{i}_{\beta}\right) R_{\beta} e\left(\boldsymbol{i}_{\beta}\right)
$$

and $e_{\beta}:=D_{\beta} \delta_{\beta}$ so that the following hypothesis is satisfied:

Hypothesis 3.9. We have:

(i) $e_{\beta}^{2}-e_{\beta} \in I_{>(\beta)}$.

(ii) $\delta_{\beta}, D_{\beta}$ and $y_{\beta}$ are $\tau$-invariant.

(iii) $\delta_{\beta} v_{\beta}^{-}=v_{\beta}^{+}$and $D_{\beta} v_{\beta}^{+}=v_{\beta}^{-}$,

(iv) $y_{\beta}$ has degree $\beta \cdot \beta$ and commutes with $\delta_{\beta}$ and $D_{\beta}$,

(v) The algebra $\left(e_{\beta} R_{\beta} e_{\beta}+I_{>(\beta)}\right) / I_{>(\beta)}$ is generated by $e_{\beta} y_{\beta} e_{\beta}+I_{>(\beta)}$.

(vi) $\iota_{\beta, \beta}\left(D_{\beta} \otimes D_{\beta}\right) \psi_{\beta, 1}=\psi_{\beta, 1} \iota_{\beta, \beta}\left(D_{\beta} \otimes D_{\beta}\right)$.

From now on until we verify it in Section 5 , we will work under the assumption that Hypothesis 3.9 holds. It turns out that this hypothesis is sufficient to construct affine cellular bases.

Lemma 3.10. $R_{\beta} e_{\beta} R_{\beta}+I_{>(\beta)}=R_{\beta}$

Proof. This follows as in the proof of Lemma 3.1 using $e_{\beta} L(\beta) \neq 0$.

Using Lemma 2.7, we can choose a set

$$
\mathfrak{B}_{\beta} \subseteq R_{\beta}
$$

of elements defined over $\mathbb{Z}$ such that

$$
\left\{b v_{\beta}^{-} \mid b \in \mathfrak{B}_{\beta}\right\}
$$

is an $\mathcal{O}$-basis of $L(\beta)_{\mathcal{O}}$.

Fix $p \in \mathbb{Z}_{>0}$ and define the set

$$
\mathfrak{B}_{\beta} \rrbracket_{p}:=\left\{\iota_{\beta, \ldots, \beta}\left(b_{1} \otimes \cdots \otimes b_{p}\right) \mid b_{1}, \ldots, b_{p} \in \mathfrak{B}_{\beta}\right\},
$$


and the element

$$
y_{\beta, r}:=\iota_{(r-1) \beta, \beta,(p-r) \beta}\left(1 \otimes y_{\beta} \otimes 1\right) \in R_{p \beta} \quad(1 \leq r \leq p) .
$$

Further, define the elements of $R_{p \beta}$

$$
\begin{aligned}
e_{\beta^{\boxplus p}} & :=\iota_{\beta, \ldots, \beta}\left(e_{\beta}, \ldots, e_{\beta}\right), \\
\delta_{\left(\beta^{p}\right)} & :=y_{\beta, 2} y_{\beta, 3}^{2} \ldots y_{\beta, p}^{p-1} \iota_{\beta, \ldots, \beta}\left(\delta_{\beta} \otimes \cdots \otimes \delta_{\beta}\right), \\
D_{\left(\beta^{p}\right)} & :=\psi_{\beta, w_{0}} \iota_{\beta, \ldots, \beta}\left(D_{\beta} \otimes \cdots \otimes D_{\beta}\right), \\
e_{\left(\beta^{p}\right)} & :=D_{\left(\beta^{p}\right)} \delta_{\left(\beta^{p}\right)}=\psi_{\beta, w_{0}} y_{\beta, 2} y_{\beta, 3}^{2} \ldots y_{\beta, p}^{p-1} e_{\beta^{\bowtie p}},
\end{aligned}
$$

where $w_{0} \in \mathfrak{S}_{p}$ is the longest element. It will be proved in Corollary 3.24 that $e_{\left(\beta^{p}\right)}^{2}-e_{\left(\beta^{p}\right)} \in I_{>\left(\beta^{p}\right)}$ generalizing part (i) of Hypothesis 3.9. It is easy to see, as in [10, Lemma 2.4], that there is always a choice of a reduced decompositon of $w_{0}$ such that

$$
\psi_{\beta, w_{0}}^{\tau}=\psi_{\beta, w_{0}}
$$

We have the algebras of polynomials and the symmetric polynomials:

$$
P_{\left(\beta^{p}\right)}=\mathcal{O}\left[y_{\beta, 1}, \ldots, y_{\beta, p}\right] \quad \text { and } \quad \Lambda_{\left(\beta^{p}\right)}=P_{\left(\beta^{p}\right)}^{\mathfrak{S}_{p}}
$$

While it is clear that the $y_{\beta, r}$ commute, we do not yet know that they are algebraically independent, but this will turn out to be the case. For now, one can interpret $\Lambda_{\left(\beta^{p}\right)}$ as the algebra generated by the elementary symmetric functions in $y_{\beta, 1}, \ldots, y_{\beta, p}$. Note using Hypothesis 3.9 (iv) that

$$
\operatorname{dim}_{q} \Lambda_{\left(\beta^{p}\right)} \leq \prod_{s=1}^{p} \frac{1}{1-q_{\beta}^{2 s}} .
$$

Given $\alpha \in Q_{+}$of height $d$ and a root partition $\pi=\left(\beta_{1}^{p_{1}}, \ldots, \beta_{N}^{p_{N}}\right) \in \Pi(\alpha)$ we define the parabolic subgroup

$$
\begin{array}{r}
\mathfrak{S}_{\pi}:=\mathfrak{S}_{\mathrm{ht}\left(\beta_{1}\right)}^{\times p_{1}} \times \cdots \times \mathfrak{S}_{\mathrm{ht}\left(\beta_{N}\right)}^{\times p_{N}} \subseteq \mathfrak{S}_{d}, \\
\mathfrak{S}_{(\pi)}:=\mathfrak{S}_{p_{1} \mathrm{ht}\left(\beta_{1}\right)} \times \cdots \times \mathfrak{S}_{p_{N} \mathrm{ht}\left(\beta_{N}\right)} \subseteq \mathfrak{S}_{d},
\end{array}
$$

and we denote by $\mathfrak{S}^{\pi}$ (resp. $\left.\mathfrak{S}^{(\pi)}\right)$ the set of minimal left coset representatives of $\mathfrak{S}_{\pi}\left(\operatorname{resp} . \mathfrak{S}_{(\pi)}\right)$ in $\mathfrak{S}_{d}$. Set

$$
\mathfrak{B}_{\pi}:=\left\{\psi_{w} \iota_{\pi}\left(b_{1} \otimes \cdots \otimes b_{N}\right) \mid w \in \mathfrak{S}^{\pi}, b_{n} \in \mathfrak{B}_{\beta_{n}^{\otimes p_{n}}} \text { for } n=1, \ldots, N\right\} .
$$

Using the natural embedding of $L\left(\beta_{1}\right)^{\bigotimes p_{1}} \otimes \cdots \otimes L\left(\beta_{N}\right)^{\bigotimes p_{N}} \subseteq \bar{\Delta}(\pi)$, we define the elements

$$
v_{\pi}^{-}=\left(v_{\beta_{1}}^{-}\right)^{\otimes p_{1}} \otimes \cdots \otimes\left(v_{\beta_{N}}^{-}\right)^{\otimes p_{N}} \in \bar{\Delta}(\pi)
$$

which belong to the word space corresponding to the words

$$
\boldsymbol{i}_{\pi}:=i_{\beta_{1}}^{p_{1}} \ldots \boldsymbol{i}_{\beta_{N}}^{p_{N}} .
$$

From definitions we have

Lemma 3.11. Let $\pi \in \Pi(\alpha)$. Then $\left\{b v_{\pi}^{-} \mid b \in \mathfrak{B}_{\pi}\right\}$ is a basis for $\bar{\Delta}(\pi)$. 
Define

$$
\begin{aligned}
\delta_{\pi} & :=\iota_{\pi}\left(\delta_{\left(\beta_{1}^{p_{1}}\right)} \otimes \cdots \otimes \delta_{\left(\beta_{N}^{p_{N}}\right)}\right), \\
D_{\pi} & :=\iota_{\pi}\left(D_{\left(\beta_{1}^{p_{1}}\right)} \otimes \cdots \otimes D_{\left(\beta_{N}^{p_{N}}\right)}\right), \\
e_{\pi} & :=\iota_{\pi}\left(e_{\left(\beta_{1}^{p_{1}}\right)} \otimes \cdots \otimes e_{\left(\beta_{N}^{p_{N}}\right)}\right)=D_{\pi} \delta_{\pi}, \\
\Delta(\pi) & :=\left(\left(R_{\alpha} e_{\pi}+I_{>\pi}\right) / I_{>\pi}\right)\left\langle\operatorname{deg}\left(v_{\pi}^{-}\right)\right\rangle, \\
\Delta^{\prime}(\pi) & :=\left(\left(e_{\pi} R_{\alpha}+I_{>\pi}\right) / I_{>\pi}\right)\left\langle\operatorname{deg}\left(v_{\pi}^{+}\right)\right\rangle, \\
\Lambda_{\pi} & :=\iota_{\pi}\left(\Lambda_{\left(\beta_{1}^{p_{1}}\right)} \otimes \cdots \otimes \Lambda_{\left(\beta_{N}^{p_{N}}\right)}\right) .
\end{aligned}
$$

Note by (3.9) and (2.26) that

$$
\operatorname{dim}_{q} \Lambda_{\pi} \leq l_{\pi}
$$

Choose also a homogeneous basis $X_{\pi}$ for $\Lambda_{\pi}$. The following lemma is a consequence of Hypothesis 3.9)(ii), (vi) and (3.7).

Lemma 3.12. We have $D_{\pi}^{\tau}=D_{\pi}$ and $\delta_{\pi}^{\tau}=\delta_{\pi}$.

3.3. Powers of a single root. Throughout this subsection $\beta \in \Phi_{+}$and $p \in \mathbb{Z}_{>0}$ are fixed. Define $\alpha:=p \beta$, and $\sigma:=\left(\beta^{p}\right) \in \Pi(\alpha)$.

Define $\bar{R}_{\alpha}:=R_{\alpha} / I_{>\sigma}$, and given $r \in R_{\alpha}$ write $\bar{r}$ for its image in $\bar{R}_{\alpha}$. The following proposition is the main result of this subsection.

Proposition 3.13. We have that

(i) $\left\{\bar{b} \bar{f} \bar{e}_{\sigma} \mid b \in \mathfrak{B}_{\sigma}, f \in X_{\sigma}\right\}$ is an $\mathcal{O}$-basis for $\Delta(\sigma)$.

(ii) $\left\{\bar{e}_{\sigma} \bar{f} \bar{D}_{\sigma} \bar{b}^{\tau} \mid b \in \mathfrak{B}_{\sigma}, f \in X_{\sigma}\right\}$ is an $\mathcal{O}$-basis for $\Delta^{\prime}(\sigma)$.

(iii) $\left\{\bar{b} \bar{e}_{\sigma} \bar{f} \bar{D}_{\sigma}\left(\bar{b}^{\prime}\right)^{\tau} \mid b, b^{\prime} \in \mathfrak{B}_{\sigma}, f \in X_{\sigma}\right\}$ is an $\mathcal{O}$-basis for $\bar{R}_{\alpha}$.

(iv) The elements $\bar{y}_{\beta, 1}, \ldots, \bar{y}_{\beta, p}$ are algebraically independent.

The proof of the Proposition will occupy this subsection. It goes by induction on $p$ ht $(\beta)$. If $\beta$ a simple root, then $R_{\alpha}=\bar{R}_{\alpha}$ is exactly the nilHecke algebra, and we are done by Theorem 2.3 . For the rest of the section, we assume the Proposition holds with $\sigma=\left(\gamma^{s}\right) \in \Pi(s \gamma)$ whenever $\gamma \in \Phi_{+}$ and $s \operatorname{ht}(\gamma)<p \operatorname{ht}(\beta)$ and prove that it also holds for $\sigma=\left(\beta^{p}\right)$. We shall also assume that $\mathcal{O}=F$ is a field, and then use Lemma 2.1 to lift to $\mathbb{Z}$-forms.

Lemma 3.14. Assume that $p=1$. Then Proposition 3.13 holds.

Proof. Since $L(\beta)$ is the unique simple module in $\bar{R}_{\beta}$-mod and

$$
\operatorname{Hom}_{\bar{R}_{\beta}}(\Delta(\beta), L(\beta))=e_{\beta} L(\beta)=F v_{\beta}^{-}
$$

is one-dimensional by Hypothesis 3.9, it follows that $\Delta(\beta)$ is the projective cover of $L(\beta)$ in $\bar{R}_{\beta}$-mod under the map $\bar{e}_{\beta} \mapsto v_{\beta}^{-}$. All composition factors of $\Delta(\beta)$ are isomorphic to $L(\beta)$. Therefore, lifting the basis $\left\{b v_{\beta}^{-} \mid b \in \mathfrak{B}_{\beta}\right\}$ of $L(\beta)$ to $\Delta(\beta)$ we see that $\Delta(\beta)$ is spanned by

$$
\left\{\bar{b} \varphi\left(\bar{e}_{\beta}\right) \mid b \in \mathfrak{B}_{\beta}, \varphi \in \operatorname{End}_{\bar{R}_{\beta}}(\Delta(\beta))\right\} .
$$


By Hypothesis 3.9 (v), $\operatorname{End}_{\bar{R}_{\beta}}(\Delta(\beta)) \simeq \bar{e}_{\beta} \bar{R}_{\beta} \bar{e}_{\beta}$ is generated by $\bar{e}_{\beta} \bar{y}_{\beta} \bar{e}_{\beta}=$ $\bar{y}_{\beta} \bar{e}_{\beta}$. Thus

$$
\Delta(\beta)=F-\operatorname{span}\left\{\bar{b} \bar{f} \bar{e}_{\beta} \mid b \in \mathfrak{B}_{\beta}, f \in X_{\beta}\right\} .
$$

Analogously, $\Delta^{\prime}(\beta)$ is the projective cover of $L(\beta)^{\tau}$ as right $\bar{R}_{\beta}$-modules under the map $\bar{e}_{\beta} \mapsto v_{\beta}^{+}$. As above, lifting the basis $\left\{v_{\beta}^{+} D_{\beta} b^{\tau} \mid b \in \mathfrak{B}_{\beta}\right\}$ of $L(\beta)^{\tau}$ to $\Delta^{\prime}(\beta)$ we see that

$$
\Delta^{\prime}(\beta)=F-\operatorname{span}\left\{\bar{e}_{\beta} \bar{f} \bar{D}_{\beta} \bar{b}^{\tau} \mid b \in \mathfrak{B}_{\beta}, f \in X_{\beta}\right\} .
$$

Therefore by Lemma 3.10 and Hypothesis 3.9 (iv),

$$
\bar{R}_{\beta}=\bar{R}_{\beta} \bar{e}_{\beta} \bar{R}_{\beta}=F-\operatorname{span}\left\{\bar{b} \bar{e}_{\beta} \bar{f} \bar{D}_{\beta}\left(\bar{b}^{\prime}\right)^{\tau} \mid b, b^{\prime} \in \mathfrak{B}_{\beta}, f \in X_{\beta}\right\} .
$$

Let $\pi=\left(\beta_{1}^{p_{1}}, \ldots, \beta_{N}^{p_{N}}\right)>(\beta)$. By definition and [7, Proposition 2.16] we have

$$
\begin{aligned}
I_{\pi} & =R_{\beta} e\left(\boldsymbol{i}_{\pi}\right) R_{\beta}+I_{>\pi} \\
& =\sum_{u, v \in \mathfrak{S}^{(\pi)}} \psi_{u} R_{\pi} e\left(\boldsymbol{i}_{\pi}\right) R_{\pi} \psi_{v}^{\tau}+I_{>\pi} \subseteq \sum_{u, v \in \mathfrak{S}^{(\pi)}} \psi_{u} R_{\pi} \psi_{v}^{\tau}+I_{>\pi},
\end{aligned}
$$

because $e\left(\boldsymbol{i}_{\pi}\right) \in R_{\pi}$. The opposite inclusion follows from Lemma 3.4 .

For $n=1, \ldots, N$, define

$$
B_{n}:=\left\{b e_{\left(\beta_{n}^{p_{n}}\right)} f D_{\beta_{n}^{p_{n}}}\left(b^{\prime}\right)^{\tau} \mid b, b^{\prime} \in \mathfrak{B}_{\left(\beta_{n}^{p_{n}}\right)}, f \in X_{\left(\beta_{n}^{p_{n}}\right)}\right\} .
$$

By part (iii) of the induction hypothesis, for $n=1, \ldots, N$, the image of $B_{n}$ in $\bar{R}_{p_{n} \beta_{n}}$ is a basis. Let

$$
B_{\pi}:=\left\{\iota_{\pi}\left(b_{1} \otimes \cdots \otimes b_{N}\right) \mid b_{1} \in \mathfrak{B}_{\left(\beta_{1}^{p_{1}}\right)}, \ldots, b_{N} \in \mathfrak{B}_{\left(\beta_{N}^{p_{N}}\right)}\right\} .
$$

By Corollary 3.6 and definitions from Section 3.2 .

$$
\begin{aligned}
R_{\pi}+I_{>\pi} & =F-\operatorname{span}\left\{\iota_{\pi}\left(r_{1} \otimes \cdots \otimes r_{N}\right) \mid r_{n} \in B_{n} \text { for } n=1, \ldots, N\right\}+I_{>\pi} \\
& =F-\operatorname{span}\left\{b e_{\pi} f D_{\pi}\left(b^{\prime}\right)^{\tau} \mid b, b^{\prime} \in B_{\pi}, f \in X_{\pi}\right\}+I_{>\pi}
\end{aligned}
$$

and therefore

$$
I_{\pi}=F-\operatorname{span}\left\{\psi_{u} b e_{\pi} f D_{\pi}\left(b^{\prime}\right)^{\tau} \psi_{v}^{\tau} \mid u, v \in \mathfrak{S}^{(\pi)}, b, b^{\prime} \in B_{\pi}, f \in X_{\pi}\right\}+I_{>\pi} .
$$

By definition of $\mathfrak{B}_{\pi}$ we have

$$
\begin{aligned}
I_{\pi} & =F-\operatorname{span}\left\{b e_{\pi} f D_{\pi}\left(b^{\prime}\right)^{\tau} \mid b, b^{\prime} \in \mathfrak{B}_{\pi}, f \in X_{\pi}\right\}+I_{>\pi}, \\
R_{\beta} & =\sum_{\pi \in \Pi(\beta)} F-\operatorname{span}\left\{b e_{\pi} f D_{\pi}\left(b^{\prime}\right)^{\tau} \mid b, b^{\prime} \in \mathfrak{B}_{\pi}, f \in X_{\pi}\right\} .
\end{aligned}
$$


Using (3.10) and the equality $\operatorname{deg}\left(D_{\pi}\right)=2 \operatorname{deg}\left(v_{\pi}^{-}\right)$for all $\pi \in \Pi(\beta)$, we get

$$
\begin{aligned}
\operatorname{dim}_{q}\left(R_{\beta}\right) & =\sum_{\pi \in \Pi(\beta)} \operatorname{dim}_{q}\left(F-\operatorname{span}\left\{b e_{\pi} f D_{\pi}\left(b^{\prime}\right)^{\tau} \mid b, b^{\prime} \in \mathfrak{B}_{\pi}, f \in X_{\pi}\right\}\right) \\
& \leq \sum_{\pi \in \Pi(\beta)}\left(\sum_{b \in \mathfrak{B}_{\pi}} q^{\operatorname{deg}(b)}\right) \operatorname{dim}_{q}\left(\Lambda_{\pi}\right) q^{\operatorname{deg}\left(D_{\pi}\right)}\left(\sum_{b \in \mathfrak{B}_{\pi}} q^{\operatorname{deg}(b)}\right) \\
& \leq \sum_{\pi \in \Pi(\beta)}\left(\sum_{b \in \mathfrak{B}_{\pi}} q^{\operatorname{deg}\left(b v_{\pi}^{-}\right)}\right)^{2} l_{\pi} \\
& =\sum_{\pi \in \Pi(\beta)} \operatorname{dim}_{q}(\bar{\Delta}(\pi))^{2} l_{\pi}=\operatorname{dim}_{q}\left(R_{\beta}\right),
\end{aligned}
$$

by Corollary 2.16. The inequalities are therefore equalities, and this implies that the spanning set $\left\{b e_{\pi} f D_{\pi}\left(b^{\prime}\right)^{\tau} \mid \pi \in \Pi(\beta), b, b^{\prime} \in \mathfrak{B}_{\pi}, f \in X_{\pi}\right\}$ of $R_{\beta}$ is a basis and $\operatorname{dim}_{q} \Lambda_{\pi}=l_{\pi}$ for all $\pi$. These yield (iii) and (iv) of Proposition 3.13 in our special case $p=1$.

To show (i) and (ii), we have already noted that the claimed bases span $\Delta(\beta)$ and $\Delta^{\prime}(\beta)$, respectively. We now apply part (iii) to see that they are linearly independent.

Corollary 3.15. We have

(i) $\bar{e}_{\beta} \bar{R}_{\beta} \bar{e}_{\beta}$ is a polynomial algebra in the variable $\bar{y}_{\beta} \bar{e}_{\beta}$.

(ii) $\Delta(\beta)$ is a free right $\bar{e}_{\beta} \bar{R}_{\beta} \bar{e}_{\beta}$-module with basis $\left\{\bar{b} \bar{e}_{\beta} \mid b \in \mathfrak{B}_{\beta}\right\}$.

(iii) $\Delta^{\prime}(\beta)$ is a free left $\bar{e}_{\beta} \bar{R}_{\beta} \bar{e}_{\beta}$-module with basis $\left\{\bar{e}_{\beta} \bar{D}_{\beta} \bar{b}^{\tau} \mid b \in \mathfrak{B}_{\beta}\right\}$.

Proof. By the lemma, we have Proposition 3.13 for $p=1$. Now, (i) follows from parts (i) and (iv) of the proposition. The remaining statements follow from parts (i) and (ii) of the proposition.

Corollary 3.16. In the Grothendieck group, we have $[\Delta(\beta)]=[L(\beta)] /(1-$ $\left.q_{\beta}^{2}\right)$.

Lemma 3.17. Up to a degree shift, $\Delta(\beta)^{\circ p} \cong \bar{R}_{p \beta} \bar{e}_{\beta} \rrbracket_{p}$.

Proof. By Corollary 3.8 we have a map

$$
\Delta(\beta)^{\boxplus p} \rightarrow \operatorname{Res}_{\beta, \ldots, \beta}\left(\bar{R}_{p \beta} \bar{e}_{\beta} \rrbracket_{p}\right), \bar{e}_{\beta}^{\otimes p} \mapsto \bar{e}_{\beta} \rrbracket_{p} .
$$

By Frobenius reciprocity, we obtain a map

$$
\mu: \Delta(\beta)^{\circ p} \rightarrow \bar{R}_{p \beta} \bar{e}_{\beta}^{\boxplus p}, 1_{\beta, \ldots, \beta} \otimes \bar{e}_{\beta}^{\otimes p} \mapsto \bar{e}_{\beta}{ }^{\boxplus p} .
$$

We now show that $I_{>\left(\beta^{p}\right)} \Delta(\beta)^{\circ p}=0$. It is enough to prove that $\operatorname{Res}_{\pi} \Delta(\beta)^{\circ p}=$ 0 for all $\pi>\left(\beta^{p}\right)$. Since all composition factors of $\Delta(\beta)$ are isomorphic to $L(\beta)$, it follows that all composition factors of $\Delta(\beta)^{\circ p}$ are isomorphic to $L(\beta)^{\circ p} \cong L\left(\beta^{p}\right)$. By Theorem 2.6 (vi), $\operatorname{Res}_{\pi}\left(L(\beta)^{\circ p}\right)=0$, which proves the claim. Since $e_{\beta}{ }_{p} 1_{\beta, \ldots, \beta} \otimes \bar{e}_{\beta}^{\otimes p}=1_{\beta, \ldots, \beta} \otimes \bar{e}_{\beta}^{\otimes p}$, we obtain a map

$$
\nu: \bar{R}_{p \beta} \bar{e}_{\beta}^{\rrbracket_{p}} \rightarrow \Delta(\beta)^{\circ p}, \bar{e}_{\beta}^{\bigotimes_{p}} \mapsto 1_{\beta, \ldots, \beta} \otimes \bar{e}_{\beta}^{\otimes p} .
$$


The homomorphisms $\mu, \nu$ map the evident cyclic generators to each other, and so are inverse isomorphisms.

Lemma 3.18. There exists an endomorphism of $\Delta(\beta) \circ \Delta(\beta)$ which sends $1_{\beta, \beta} \otimes\left(\bar{e}_{\beta} \otimes \bar{e}_{\beta}\right)$ to $\psi_{\beta, 1} 1_{\beta, \beta} \otimes\left(\bar{e}_{\beta} \otimes \bar{e}_{\beta}\right)$.

Proof. Apply the Mackey theorem to $\operatorname{Res}_{\beta, \beta}(\Delta(\beta) \circ \Delta(\beta))$. We get a short exact sequence of $R_{\beta} \otimes R_{\beta}$-modules

$0 \rightarrow \Delta(\beta) \otimes \Delta(\beta) \rightarrow \operatorname{Res}_{\beta, \beta}(\Delta(\beta) \circ \Delta(\beta)) \rightarrow(\Delta(\beta) \otimes \Delta(\beta))\langle-\beta \cdot \beta\rangle \rightarrow 0$,

where $\psi_{\beta, 1} 1_{\beta, \beta} \otimes\left(\bar{e}_{\beta} \otimes \bar{e}_{\beta}\right) \in \operatorname{Res}_{\beta, \beta}(\Delta(\beta) \circ \Delta(\beta))$ is a preimage of the standard generator of $(\Delta(\beta) \otimes \Delta(\beta))\langle-\beta \cdot \beta\rangle$.

We now show that this is actually a sequence of $\bar{R}_{\beta} \otimes \bar{R}_{\beta}$-modules. It is sufficient to show that for any $\pi>(\beta)$, we have that

$$
\operatorname{Res}_{\pi, \beta} \circ \operatorname{Res}_{\beta, \beta}(\Delta(\beta) \circ \Delta(\beta))=0=\operatorname{Res}_{\beta, \pi} \circ \operatorname{Res}_{\beta, \beta}(\Delta(\beta) \circ \Delta(\beta)) .
$$

We show the first equality, the second being similar. All composition factors of $\Delta(\beta)$ are isomorphic to $L(\beta)$, so all composition factors of $\Delta(\beta) \circ$ $\Delta(\beta)$ are isomorphic to $L(\beta) \circ L(\beta)$, and thus all composition factors of $\operatorname{Res}_{\beta, \beta}(\Delta(\beta) \circ \Delta(\beta))$ are isomorphic to $L(\beta) \otimes L(\beta)$. Theorem 2.6 now tells us that $\operatorname{Res}_{\pi}(L(\beta))=0$ for all $\pi>(\beta)$.

By the projectivity of $\Delta(\beta)$ as $\bar{R}_{\beta}$-module, the short exact sequence splits, giving the required endomorphism by Frobenius reciprocity.

Corollary 3.19. $\bar{\psi}_{\beta, 1} \bar{e}_{\beta^{\boxplus 2}}=\bar{e}_{\beta}{ }^{\bigotimes 2} \bar{\psi}_{\beta, 1} \bar{e}_{\beta}{ }^{\boxplus 2}$.

Proof. Let $\varphi$ be the endomorphism of $\Delta(\beta) \circ \Delta(\beta)$ constructed in Lemma 3.18 , regarded as an endomorphism of $\bar{R}_{2 \beta} \bar{e}_{\beta}{ }^{\bowtie 2}$ by Lemma 3.17. Then

$$
\bar{\psi}_{\beta, 1} \bar{e}_{\beta} \beta_{2}=\varphi\left(\bar{e}_{\beta}{ }^{\boxplus 2}\right)=\varphi\left(\bar{e}_{\beta}^{2} 2\right)=\bar{e}_{\beta}{ }^{\boxplus 2} \varphi\left(\bar{e}_{\beta}{ }^{\boxplus 2}\right)=\bar{e}_{\beta}{ }^{\boxplus 2} \bar{\psi}_{\beta, 1} \bar{e}_{\beta}{ }^{\boxplus 2},
$$

as required.

Corollary 3.20. We have $\bar{e}_{\beta^{\boxplus p}} \bar{e}_{\left(\beta^{p}\right)} \bar{e}_{\beta^{\boxplus p}}=\bar{e}_{\left(\beta^{p}\right)}$.

Proof. Follows from (3.6), Corollary 3.19 and Hypothesis 3.9 .

Lemma 3.21. The set $\left\{\bar{e}_{\beta} \rrbracket_{p} \bar{y}_{\beta, 1}^{a_{1}} \ldots \bar{y}_{\beta, p}^{a_{p}} \bar{\psi}_{\beta, w} \bar{e}_{\beta} \rrbracket_{p} \mid w \in \mathfrak{S}_{p}, a_{1}, \ldots, a_{p} \geq 0\right\}$ gives a linear basis of $\bar{e}_{\beta}{ }^{{ }_{p}} \bar{R}_{\alpha} \bar{e}_{\beta}{ }^{{ }_{1}}$.

Proof. The elements above are linearly independent by Lemmas 3.17 and 3.18, and Corollary 3.15. We use Frobenius reciprocity, Corollary 3.16, and [2, Lemma 2.11] to see that

$$
\begin{aligned}
\operatorname{dim}_{q} \operatorname{End}_{R_{\alpha}}\left(\Delta(\beta)^{\circ p}\right) & =\operatorname{dim}_{q} \operatorname{Hom}_{R_{\beta, \ldots, \beta}}\left(\Delta(\beta)^{\bowtie p}, \operatorname{Res}_{\beta, \ldots, \beta} \Delta(\beta)^{\circ p}\right) \\
& \leq\left[\operatorname{Res}_{\beta, \ldots, \beta} \Delta(\beta)^{\circ p}: L(\beta)^{\bowtie p}\right] \\
& =\left[\operatorname{Res}_{\beta, \ldots, \beta} L(\beta)^{\circ p}: L(\beta)^{\bowtie p}\right] /\left(1-q_{\beta}^{2}\right)^{p} \\
& =q_{\beta}^{-\frac{1}{2} p(p-1)}[p]_{\beta}^{!} /\left(1-q_{\beta}^{2}\right)^{p} .
\end{aligned}
$$


By the formula for the Poincaré polynomial of $\mathfrak{S}_{p}$, we have shown that

$$
\operatorname{dim}_{q} \bar{e}_{\beta^{\bowtie p}} \bar{R}_{\alpha} \bar{e}_{\beta^{\boxplus p}} \leq \frac{\sum_{w \in \mathfrak{S}_{p}} q_{\beta}^{-2 l(w)}}{\left(1-q_{\beta}^{2}\right)^{p}},
$$

showing that the proposed basis also spans.

The next two lemmas are proved using ideas that already appeared in the proofs of [2, Lemmas].

Lemma 3.22. We have that

$$
\begin{aligned}
& \bar{\psi}_{\beta, r}^{2} \bar{e}_{\beta \boxplus p}=0, \quad \text { for } 1 \leq r \leq p-1, \\
& \bar{\psi}_{\beta, r} \bar{\psi}_{\beta, s} \bar{e}_{\beta} \rrbracket_{p}=\bar{\psi}_{\beta, s} \bar{\psi}_{\beta, r} \bar{e}_{\beta} \rrbracket_{p}, \quad \text { for }|r-s|>1 \text {, and } \\
& \bar{\psi}_{\beta, r} \bar{\psi}_{\beta, r+1} \bar{\psi}_{\beta, r} \bar{e}_{\beta}{ }^{\boxplus p}=\bar{\psi}_{\beta, r+1} \bar{\psi}_{\beta, r} \bar{\psi}_{\beta, r+1} \bar{e}_{\beta}{ }^{\boxplus p}, \quad \text { for } 1 \leq r \leq p-2 .
\end{aligned}
$$

Proof. We use Lemma 3.17 to identify $\bar{R}_{p \beta} \bar{e}_{\beta} \otimes_{p}$ with $\Delta(\beta)^{\circ p}$. It is enough to prove the first relation in the case $p=2$. The Mackey theorem analysis in the proof of Lemma 3.18 shows that, as a graded vector space

$$
(\Delta(\beta) \circ \Delta(\beta))_{i_{\beta}^{2}}=e\left(i_{\beta}^{2}\right) \otimes(\Delta(\beta) \otimes \Delta(\beta)) \oplus \psi_{\beta, 1} e\left(i_{\beta}^{2}\right) \otimes(\Delta(\beta) \otimes \Delta(\beta)) .
$$

The vector $\bar{e}_{\beta} \in \Delta(\beta)_{\boldsymbol{i}_{\beta}}$ is of minimal degree, and thus $\psi_{\beta, 1} e\left(\boldsymbol{i}_{\beta}^{2}\right) \otimes\left(\bar{e}_{\beta} \otimes \bar{e}_{\beta}\right)$ is of minimal degree in $(\Delta(\beta) \circ \Delta(\beta))_{i_{\beta}^{2}}$. The degree of $\psi_{\beta, 1}^{2} e\left(\boldsymbol{i}_{\beta}^{2}\right) \otimes\left(\bar{e}_{\beta} \otimes \bar{e}_{\beta}\right)$ is smaller by $\beta \cdot \beta$, so the vector is zero.

The second relation is clear from the definitions. To prove the third relation, it is sufficient to consider $p=3$. Let $w_{r}:=w_{\beta, r}$, and set $w_{0}:=$ $w_{1} w_{2} w_{1}$. Using the defining relations of $R_{3 \beta}$, we deduce that $\left(\psi_{\beta, 2} \psi_{\beta, 1} \psi_{\beta, 2}-\right.$ $\left.\psi_{\beta, 1} \psi_{\beta, 2} \psi_{\beta, 1}\right) e\left(\boldsymbol{i}_{\beta}^{3}\right) \otimes\left(\bar{e}_{\beta} \otimes \bar{e}_{\beta} \otimes \bar{e}_{\beta}\right)$ is an element of degree $3 \operatorname{deg}\left(v_{\beta}^{-}\right)-6 \beta \cdot \beta$ in $S:=\sum_{w<w_{0}} \psi_{w} e\left(i_{\beta}^{3}\right) \otimes(\Delta(\beta) \otimes \Delta(\beta) \otimes \Delta(\beta))$, where $<$ denotes the Bruhat order. By a Mackey theorem analysis as in the proof of Lemma 3.18, we see that

$$
S=\sum_{w \in\left\{1, w_{1}, w_{2}, w_{1} w_{2}, w_{2} w_{1}\right\}} \psi_{w} e\left(i_{\beta}^{3}\right) \otimes(\Delta(\beta) \otimes \Delta(\beta) \otimes \Delta(\beta)) .
$$

The lowest degree of an element in $S$ is therefore $3 \operatorname{deg}\left(v_{\beta}^{-}\right)-4 \beta \cdot \beta$, and the third relation is proved.

Lemma 3.23. There exists a unique choice of $\varepsilon_{\beta}= \pm 1$ such that

$$
\begin{aligned}
& \bar{\psi}_{\beta, r} \bar{y}_{\beta, s} \bar{e}_{\beta{ } p}=\bar{y}_{\beta, s} \bar{\psi}_{\beta, r} \bar{e}_{\beta{ }_{p}}, \\
& \text { for } s \neq r, r+1 \text {, } \\
& \bar{\psi}_{\beta, r} \varepsilon_{\beta} \bar{y}_{\beta, r+1} \bar{e}_{\beta} \rrbracket_{p}=\left(\varepsilon_{\beta} \bar{y}_{\beta, r} \bar{\psi}_{\beta, r}+1\right) \bar{e}_{\beta} \rrbracket_{p}, \\
& \text { for } 1 \leq r<p \text {, and } \\
& \varepsilon_{\beta} \bar{y}_{\beta, r+1} \bar{\psi}_{\beta, r} \bar{e}_{\beta} \rrbracket_{p}=\left(\bar{\psi}_{\beta, r} \varepsilon_{\beta} \bar{y}_{\beta, r}+1\right) \bar{e}_{\beta} \rrbracket_{p}, \\
& \text { for } 1 \leq r<p \text {. }
\end{aligned}
$$

Proof. The first relation is clear from the definitions. It is enough to prove the remaining relations for $p=2$. Using the defining relations of $R_{2 \beta}$ and a 
Mackey theorem analysis as in the proof of Lemma 3.18, we deduce that

$$
\begin{aligned}
\left(\bar{\psi}_{\beta, 1} \bar{y}_{\beta, 2}-\bar{y}_{\beta, 1} \bar{\psi}_{\beta, 1}\right) \bar{e}_{\beta}{ }_{\beta 2} & \in \sum_{w<w_{\beta, 1}} \psi_{w} e\left(\boldsymbol{i}_{\beta}^{2}\right) \otimes(\Delta(\beta) \otimes \Delta(\beta)) \\
& =e\left(\boldsymbol{i}_{\beta}^{2}\right) \otimes(\Delta(\beta) \otimes \Delta(\beta)),
\end{aligned}
$$

and the only vector of the correct degree is $\bar{e}_{\beta} \otimes_{2}$. Therefore (working over $\mathbb{Z})$ we must have that

$$
\left(\bar{\psi}_{\beta, 1} \bar{y}_{\beta, 2}-\bar{y}_{\beta, 1} \bar{\psi}_{\beta, 1}\right) \bar{e}_{\beta}{ }^{\bowtie 2}=c_{+} \bar{e}_{\beta}{ }^{\bowtie 2}
$$

for some $c_{+} \in \mathbb{Z}$. Similarly, we obtain

$$
\left(\bar{\psi}_{\beta, 1} \bar{y}_{\beta, 1}-\bar{y}_{\beta, 2} \bar{\psi}_{\beta, 1}\right) \bar{e}_{\beta}{ }^{\bowtie 2}=c_{-} \bar{e}_{\beta}{ }^{\boxplus 2}
$$

for some $c_{-} \in \mathbb{Z}$. We compute

$$
\begin{aligned}
\left(\bar{\psi}_{\beta, 1} \bar{y}_{\beta, 1} \bar{y}_{\beta, 2}-\bar{y}_{\beta, 1} \bar{y}_{\beta, 2} \bar{\psi}_{\beta, 1}\right) \bar{e}_{\beta \boxplus 2} & =\left(\bar{y}_{\beta, 2} \bar{\psi}_{\beta, 1}+c_{-}\right) \bar{y}_{\beta, 2}-\bar{y}_{\beta, 2}\left(\bar{\psi}_{\beta, 1} \bar{y}_{\beta, 2}-c_{+}\right) \\
& =\left(c_{-}+c_{+}\right) \bar{y}_{\beta, 2} \bar{e}_{\beta}{ }^{\boxplus 2} \\
\left(\bar{\psi}_{\beta, 1} \bar{y}_{\beta, 1} \bar{y}_{\beta, 2}-\bar{y}_{\beta, 1} \bar{y}_{\beta, 2} \bar{\psi}_{\beta, 1}\right) \bar{e}_{\beta}{ }^{\boxplus 2} & =\left(\bar{y}_{\beta, 1} \bar{\psi}_{\beta, 1}+c_{+}\right) \bar{y}_{\beta, 1}-\bar{y}_{\beta, 1}\left(\bar{\psi}_{\beta, 1} \bar{y}_{\beta, 1}-c_{-}\right) \\
& =\left(c_{+}+c_{-}\right) \bar{y}_{\beta, 1} \bar{e}_{\beta}{ }^{\boxplus 2}
\end{aligned}
$$

and since $\bar{y}_{\beta, 1} \bar{e}_{\beta \bowtie 2}$ and $\bar{y}_{\beta, 1} \bar{e}_{\beta \bowtie 2}$ are linearly independent by Lemma 3.21, we must have $c_{-}=-c_{+}$. We now fix a prime $p$ and extend scalars to $\mathbb{F}_{p}$. Suppose that $\varepsilon_{\beta}=0 \in \mathbb{F}_{p}$, so that

$$
\begin{aligned}
& \bar{\psi}_{\beta, 1} \bar{y}_{\beta, 2} \bar{e}_{\beta}{ }^{\boxplus 2}=\bar{y}_{\beta, 1} \bar{\psi}_{\beta, 1} \bar{e}_{\beta}{ }^{\boxplus 2} \\
& \bar{\psi}_{\beta, 1} \bar{y}_{\beta, 1} \bar{e}_{\beta}{ }^{\boxplus 2} \\
& =\bar{y}_{\beta, 2} \bar{\psi}_{\beta, 1} \bar{e}_{\beta}{ }^{\boxplus 2} .
\end{aligned}
$$

Define $S$ to be the submodule of $\Delta(\beta) \circ \Delta(\beta)$ generated by $\bar{y}_{\beta, 1} \bar{e}_{\beta}{ }^{\otimes 2}$ and $\bar{y}_{\beta, 2} \bar{e}_{\beta} \otimes_{2}$. The above equations show that the endomorphism defined by right multiplication by $\bar{\psi}_{\beta, 1} \bar{e}_{\beta}{ }^{\boxplus 2}$ leaves $S$ invariant. On the other hand, $\Delta(\beta) \circ$ $\Delta(\beta) / S \cong L(\beta) \circ L(\beta)$ is irreducible. Since the endomorphism algebra of an irreducible module is one dimensional, we have a contradiction. Therefore $\varepsilon_{\beta} \neq 0$ when reduced modulo any prime, i.e. $\varepsilon_{\beta}= \pm 1$.

Corollary 3.24. The homomorphism from the nilHecke algebra $H_{p}$ determined by

$$
\zeta: H_{p} \rightarrow \bar{e}_{\beta{ }_{p}} \bar{R}_{\alpha} \bar{e}_{\beta} \rrbracket_{p}, y_{r} \mapsto \varepsilon_{\beta} \bar{y}_{\beta, r} \bar{e}_{\beta} \rrbracket_{p}, \psi_{r} \mapsto \bar{\psi}_{\beta, r} \bar{e}_{\beta} \rrbracket_{p}
$$

is an isomorphism. Under this isomorphism the idempotent $e_{p} \in H_{p}$ is mapped onto $\bar{e}_{\sigma}$.

Proof. Using Lemmas 3.22 and 3.23, we see that the map exists. By Lemma 3.21, the map is an isomorphism. The second statement now follows using Corollary 3.20 .

Corollary 3.25. Given $f \in \Lambda_{\sigma}, \bar{f}$ commutes with $\bar{\delta}_{\sigma}, \bar{e}_{\sigma}$, and $\bar{e}_{\sigma} \bar{D}_{\sigma}$. 
Proof. It follows directly from Hypothesis 3.9)(iv) and the definitions that $\delta_{\sigma}$ commutes with every element of $P_{\sigma}$, and in particular with every element of the subalgebra $\Lambda_{\sigma}$. Denote by $w_{0}$ the longest element of $\mathfrak{S}_{p}$. Then by Corollaries 3.19 and 3.24

$$
\begin{aligned}
\bar{e}_{\sigma} \bar{D}_{\sigma} & =\bar{\psi}_{\beta, w_{0}} \bar{y}_{\beta, 2} \ldots \bar{y}_{\beta, p}^{p-1} \bar{e}_{\beta}{ }^{\boxplus p} \bar{\psi}_{\beta, w_{0}} \iota\left(D_{\beta} \otimes \cdots \otimes D_{\beta}\right) \\
& =\left(\bar{e}_{\beta} \otimes_{p} \psi_{\beta, w_{0}} \bar{e}_{\beta} \otimes_{p}\right)\left(\bar{y}_{\beta, 2} \ldots \bar{y}_{\beta, p}^{p-1}\right)\left(\bar{e}_{\beta{ }} \psi_{\beta, w_{0}} \bar{e}_{\beta}{ }_{\beta p}\right) \iota\left(D_{\beta} \otimes \cdots \otimes D_{\beta}\right) \\
& =\zeta\left(\psi_{w_{0}}\right)\left(\bar{y}_{\beta, 2} \ldots \bar{y}_{\beta, p}^{p-1}\right) \zeta\left(\psi_{w_{0}}\right) \iota\left(D_{\beta} \otimes \cdots \otimes D_{\beta}\right)
\end{aligned}
$$

Any $f \in \Lambda_{\sigma}$ commutes with $\iota\left(D_{\beta} \otimes \cdots \otimes D_{\beta}\right)$ by Hypothesis [3.9)(iv). It is well known that the center of the nilHecke algebra $H_{p}$ is given by the symmetric functions $\Lambda_{p}$. In particular, every element of $\Lambda_{p}$ commutes with $\psi_{w_{0}}$. Let $g \in \Lambda_{p}$ be such that $\zeta(g)=\bar{f} \bar{e}_{\beta} \bigotimes_{p}$. Then $\zeta\left(\psi_{w_{0}}\right) \bar{f}=\zeta\left(\psi_{w_{0}} g\right)=\zeta\left(g \psi_{w_{0}}\right)=$ $f \zeta\left(\psi_{w_{0}}\right)$. This implies the claim.

We can now finish the proof of Proposition 3.13. Corollary 3.24 provides an isomorphism $H_{p} \cong \operatorname{End}_{R_{\alpha}}\left(\bar{R}_{\alpha} \bar{e}_{\beta} \otimes_{p}\right)$ under which the idempotent $e_{p}$ corresponds to right multiplication by $\bar{e}_{\sigma}$. But $e_{p}$ is a primitive idempotent, so the image $\bar{R}_{\alpha} \bar{e}_{\sigma}=\Delta(\sigma)$ of this endomorphism is an indecomposable projective $\bar{R}_{\alpha}$-module. We may identify

$$
\operatorname{End}_{R_{\alpha}}(\Delta(\sigma)) \cong \bar{e}_{\sigma} \bar{R}_{\alpha} \bar{e}_{\sigma}=\zeta\left(e_{p} H_{p} e_{p}\right)=\zeta\left(\Lambda_{p} e_{p}\right)=\bar{e}_{\sigma} \bar{\Lambda}_{\sigma} \bar{e}_{\sigma} \cong \Lambda_{\sigma}
$$

where the action of $\Lambda_{\sigma}$ on $\Delta(\sigma)=\bar{R}_{\alpha} \bar{e}_{\sigma}$ is given by right multiplication which makes sense in view of Corollary 3.25. Therefore $\Delta(\sigma) \rightarrow L(\sigma), \bar{e}_{\sigma} \mapsto$ $v_{\sigma}^{-}$is a projective cover in $\bar{R}_{\alpha}$-mod. Furthermore, since $\bar{R}_{\alpha}$-mod has only one irreducible module, every composition factor of $\Delta(\sigma)$ is isomorphic to $L(\sigma)$ with an appropriate degree shift. We can lift the basis $\left\{b v_{\sigma}^{-} \mid b \in \mathfrak{B}_{\sigma}\right\}$ for $L(\sigma)$ to the set $\left\{\bar{b} \bar{e}_{\sigma} \mid b \in \mathfrak{B}_{\sigma}\right\} \subseteq \Delta(\sigma)$. Using the basis $X_{\sigma}$ for $\Lambda_{\sigma}$, we get a basis $\left\{\bar{b} \bar{f} \bar{e}_{\sigma} \mid b \in \mathfrak{B}_{\sigma}, f \in X_{\sigma}\right\}$ for $\Delta(\sigma)$.

Similarly, $\Delta^{\prime}(\sigma) \rightarrow L(\sigma)^{\tau}, \bar{e}_{\sigma} \mapsto v_{\sigma}^{+}$is a projective cover in $\bar{R}_{\alpha}^{o p}$-mod. It is immediate that $\left\{v_{\sigma}^{+} D_{\sigma} b^{\tau} \mid b \in \mathfrak{B}_{\sigma}\right\}$ is a basis of $L(\sigma)^{\tau}$. Lifting as above, we have that $\left\{\bar{e}_{\sigma} \bar{f} \bar{D}_{\sigma} \bar{b}^{\tau} \mid b \in \mathfrak{B}_{\sigma}, f \in X_{\sigma}\right\}$ is a basis for $\Delta^{\prime}(\sigma)$.

Finally, applying the multiplication map and Corollary 3.25 we have that $\left.\left\{\bar{b} \bar{e}_{\sigma} \bar{f} \bar{D}_{\sigma} \overline{ }^{\prime} b^{\prime}\right)^{\tau} \mid b, b^{\prime} \in \mathfrak{B}_{\sigma}, f \in X_{\sigma}\right\}$ spans $\bar{R}_{\alpha}$. Therefore by induction,

$$
\left.R_{\alpha}=F-\operatorname{span}\left\{\bar{b} \bar{e}_{\pi} \bar{f} \bar{D}_{\pi} \overline{(} b^{\prime}\right)^{\tau} \mid \pi \in \Pi(\alpha), b, b^{\prime} \in \mathfrak{B}_{\pi}, f \in X_{\pi}\right\},
$$

and comparing graded dimensions with Corollary 2.16 as in the proof of Lemma 3.14, this set is therefore a basis.

3.4. General case. In this section we use the results of the previous subsections to obtain affine cellular bases of the KLR algebras of finite type. Fix $\alpha \in Q_{+}$and $\pi=\left(\beta_{1}^{p_{1}}, \ldots, \beta_{N}^{p_{N}}\right) \in \Pi(\alpha)$. Define $\bar{R}_{\alpha}:=R_{\alpha} / I_{>\pi}$, and write $\bar{r} \in \bar{R}_{\alpha}$ for the image of an element $r \in R_{\alpha}$.

We begin with some easy consequences of the previous section.

Corollary 3.26. We have

(i) Given $f \in \Lambda_{\pi}, \bar{f}$ commutes with $\bar{\delta}_{\pi}, \bar{e}_{\pi}$, and $\bar{e}_{\pi} \bar{D}_{\pi}$. 
(ii) Up to a grading shift, $\Delta(\pi) \cong \Delta\left(\beta_{1}^{p_{1}}\right) \circ \cdots \circ \Delta\left(\beta_{N}^{p_{N}}\right)$.

(iii) The map $\Lambda_{\pi} \rightarrow \operatorname{End}_{\bar{R}_{\alpha}}(\Delta(\pi))$ sending $f$ to right multiplication by $\bar{e}_{\pi} \bar{f} \bar{e}_{\pi}$ is an isomorphism of algebras.

(iv) The map $\Lambda_{\pi} \rightarrow \operatorname{End}_{\bar{R}_{\alpha}}\left(\Delta^{\prime}(\pi)\right)$ sending $f$ to left multiplication by $\bar{e}_{\pi} \bar{f} \bar{e}_{\pi}$ is an isomorphism of algebras.

Proof. Claim (i) follows directly from Corollary 3.25 and the definitions.

The proof of claim (ii) is similar to that of Lemma 3.17. To be precise, by Corollary 3.6 we have a map

$$
\Delta\left(\beta_{1}^{p_{1}}\right) \otimes \cdots \otimes \Delta\left(\beta_{N}^{p_{N}}\right) \rightarrow \operatorname{Res}_{\pi} \Delta(\pi), \bar{e}_{\left(\beta_{1}^{p_{1}}\right)} \otimes \cdots \otimes \bar{e}_{\left(\beta_{N}^{p_{N}}\right)} \mapsto \bar{e}_{\pi},
$$

which by Frobenius reciprocity determines a homomorphism

$$
\mu: \Delta\left(\beta_{1}^{p_{1}}\right) \circ \cdots \circ \Delta\left(\beta_{N}^{p_{N}}\right) \rightarrow \Delta(\pi), 1_{\pi} \otimes\left(\bar{e}_{\left(\beta_{1}^{p_{1}}\right)} \otimes \cdots \otimes \bar{e}_{\left(\beta_{N}^{p_{N}}\right)}\right) \mapsto \bar{e}_{\pi} .
$$

We now claim that $I_{>\pi}\left(\Delta\left(\beta_{1}^{p_{1}}\right) \circ \cdots \circ \Delta\left(\beta_{N}^{p_{N}}\right)\right)=0$. It is enough to prove that $\operatorname{Res}_{\sigma}\left(\Delta\left(\beta_{1}^{p_{1}}\right) \circ \cdots \circ \Delta\left(\beta_{N}^{p_{N}}\right)\right)=0$ for all $\sigma>\pi$. By exactness of induction, it follows that $\Delta\left(\beta_{1}^{p_{1}}\right) \circ \cdots \circ \Delta\left(\beta_{N}^{p_{N}}\right)$ has an exhaustive filtration by $L\left(\beta_{1}^{p_{1}}\right) \circ \cdots \circ L\left(\beta_{N}^{p_{N}}\right)=\bar{\Delta}(\pi)$. By Theorem $\left[2.6(\right.$ vi $), \operatorname{Res}_{\sigma}(\bar{\Delta}(\pi))=0$, which proves the claim.

Since

$$
e_{\pi} 1_{\pi} \otimes\left(\bar{e}_{\left(\beta_{1}^{p_{1}}\right)} \otimes \cdots \otimes \bar{e}_{\left(\beta_{N}^{p_{N}}\right)}\right)=1_{\pi} \otimes\left(\bar{e}_{\left(\beta_{1}^{p_{1}}\right)} \otimes \cdots \otimes \bar{e}_{\left(\beta_{N}^{p_{N}}\right)}\right),
$$

we obtain a map

$$
\nu: \Delta(\pi) \rightarrow \Delta\left(\beta_{1}^{p_{1}}\right) \circ \cdots \circ \Delta\left(\beta_{N}^{p_{N}}\right), \bar{e}_{\pi} \mapsto 1_{\pi} \otimes\left(\bar{e}_{\left(\beta_{1}^{p_{1}}\right)} \otimes \cdots \otimes \bar{e}_{\left(\beta_{N}^{p_{N}}\right)}\right) .
$$

The homomorphisms $\mu, \nu$ map the evident cyclic generators to each other, and so are inverse isomorphisms.

We use claim (ii) to identify $\Delta(\pi)$ with $\Delta\left(\beta_{1}^{p_{1}}\right) \circ \ldots \Delta\left(\beta_{N}^{p_{N}}\right)$. As noted in the proof of claim (ii), $\Delta(\pi)$ has an exhaustive filtration by

$$
\bar{\Delta}(\pi)=\oplus_{w \in \mathfrak{S}^{(\pi)}} \psi_{w} 1_{\pi} \otimes\left(\bar{\Delta}\left(\beta_{1}^{p_{1}}\right) \otimes \cdots \otimes \bar{\Delta}\left(\beta_{N}^{p_{N}}\right)\right) .
$$

By Theorem 2.6(vi), $\operatorname{Res}_{\pi} \bar{\Delta}(\pi)$ picks out the summand corresponding to $w=1$. Therefore $\operatorname{Res}_{\pi} \Delta(\pi) \cong \Delta\left(\beta_{1}^{p_{1}}\right) \otimes \cdots \otimes \Delta\left(\beta_{N}^{p_{N}}\right)$. Applying Frobenius reciprocity and (3.14), we obtain

$$
\begin{aligned}
\operatorname{End}_{R_{\alpha}}(\Delta(\pi)) & =\operatorname{Hom}_{R_{\alpha}}\left(\Delta\left(\beta_{1}^{p_{1}}\right) \circ \cdots \circ \Delta\left(\beta_{n}^{p_{N}}\right), \Delta\left(\beta_{1}^{p_{1}}\right) \circ \cdots \circ \Delta\left(\beta_{n}^{p_{N}}\right)\right) \\
& \simeq \operatorname{Hom}_{R_{\pi}}\left(\Delta\left(\beta_{1}^{p_{1}}\right) \otimes \cdots \otimes \Delta\left(\beta_{n}^{p_{N}}\right), \operatorname{Res}_{\pi}\left(\Delta\left(\beta_{1}^{p_{1}}\right) \circ \cdots \circ \Delta\left(\beta_{n}^{p_{N}}\right)\right)\right) \\
& \simeq \operatorname{End}_{R_{\pi}}\left(\Delta\left(\beta_{1}^{p_{1}}\right) \otimes \cdots \otimes \Delta\left(\beta_{N}^{p_{N}}\right)\right) \\
& \simeq \operatorname{End}_{R_{p_{1} \beta_{1}}}\left(\Delta\left(\beta_{1}^{p_{1}}\right)\right) \otimes \cdots \otimes \operatorname{End}_{R_{p_{1} \beta_{N}}}\left(\Delta\left(\beta_{1}^{p_{N}}\right)\right) \\
& \simeq \Lambda_{\left(\beta_{1}^{p_{1}}\right)} \otimes \cdots \otimes \Lambda_{\left(\beta_{N}^{p_{N}}\right)} \simeq \Lambda_{\pi} .
\end{aligned}
$$

This proves claim (iii), and claim (iv) is shown similarly.

Proposition 3.27. We have that

(i) $\left\{\bar{b} \bar{f} \bar{e}_{\pi} \mid b \in \mathfrak{B}_{\pi}, f \in X_{\pi}\right\}$ is an $\mathcal{O}$-basis for $\Delta(\pi)$, 
(ii) $\left\{\bar{e}_{\pi} \bar{f} \bar{D}_{\pi} \bar{b}^{\tau} \mid b \in \mathfrak{B}_{\pi}, f \in X_{\pi}\right\}$ is an $\mathcal{O}$-basis for $\Delta^{\prime}(\pi)$, and

(iii) $\left\{\bar{b} \bar{e}_{\pi} \bar{f} \bar{D}_{\pi}\left(\bar{b}^{\prime}\right)^{\tau} \mid b, b^{\prime} \in \mathfrak{B}_{\pi}, f \in X_{\pi}\right\}$ is an $\mathcal{O}$-basis for $\bar{I}_{\pi}$.

Proof. For $n=1, \ldots, N$, define

$$
B_{n}:=\left\{\bar{b} \bar{f} \bar{e}_{\left(\beta_{n}^{p_{n}}\right)} \mid b \in \mathfrak{B}_{\left(\beta_{n}^{p_{n}}\right)}, f \in X_{\left(\beta_{n}^{p_{n}}\right)}\right\} .
$$

By Proposition 3.13, $B_{n}$ is a basis of $\Delta\left(\beta_{n}^{p_{n}}\right)$ for each $n=1, \ldots, N$. Let $\bar{\iota}_{\pi}: \bar{R}_{p_{1} \beta_{1}} \otimes \cdots \otimes \bar{R}_{p_{N} \beta_{N}} \rightarrow \bar{R}_{\alpha}$ be the map induced by $\iota_{\pi}$, as in Corollary 3.6 , Using [7, Proposition 2.16], and computing as in the proof of Lemma 3.14, we have

$$
\begin{aligned}
\Delta(\pi) & =\sum_{w \in \mathfrak{S}^{(\pi)}} \bar{\psi}_{w} \bar{R}_{\pi} \bar{e}_{\pi}=\sum_{w \in \mathfrak{S}^{(\pi)}} \bar{\psi}_{w} \bar{\iota}_{\pi}\left(\Delta\left(\beta_{1}^{p_{1}}\right) \otimes \cdots \otimes \Delta\left(\beta_{N}^{p_{N}}\right)\right) \\
& =\mathcal{O}-\operatorname{span}\left\{\bar{\psi}_{w} \bar{\iota}_{\pi}\left(b_{1} \otimes \cdots \otimes b_{N}\right) \mid w \in \mathfrak{S}^{(\pi)}, b_{n} \in B_{n}\right\} \\
& =\mathcal{O}-\operatorname{span}\left\{\bar{b} \bar{f} \bar{e}_{\pi} \mid b \in \mathfrak{B}_{\pi}, f \in X_{\pi}\right\} .
\end{aligned}
$$

We have shown that the set in (i) spans $\Delta(\pi)$. A similar argument shows that the set in (ii) spans $\Delta^{\prime}(\pi)$. Now, applying the multiplication map $\Delta(\pi) \otimes \Delta^{\prime}(\pi) \rightarrow \bar{I}_{\pi}$ and using Corollary $3.26(\mathrm{i})$ yields the spanning set of (iii). Letting $\pi$ vary over $\Pi(\alpha)$, we have

$$
R_{\alpha}=\sum_{\pi \in \Pi(\alpha)} \mathcal{O}-\operatorname{span}\left\{b e_{\pi} f D_{\pi}\left(b^{\prime}\right)^{\tau} \mid b, b^{\prime} \in \mathfrak{B}_{\pi}, f \in X_{\pi}\right\} .
$$

Using (3.10) and the equality $\operatorname{deg}\left(D_{\pi}\right)=2 \operatorname{deg}\left(v_{\pi}^{-}\right)$for all $\pi \in \Pi(\alpha)$, we get

$$
\begin{aligned}
\operatorname{dim}_{q}\left(R_{\alpha}\right) & =\sum_{\pi \in \Pi(\alpha)} \operatorname{dim}_{q}\left(\mathcal{O}-\operatorname{span}\left\{b e_{\pi} f D_{\pi}\left(b^{\prime}\right)^{\tau} \mid b, b^{\prime} \in \mathfrak{B}_{\pi}, f \in X_{\pi}\right\}\right) \\
& \leq \sum_{\pi \in \Pi(\alpha)}\left(\sum_{b \in \mathfrak{B}_{\pi}} q^{\operatorname{deg}(b)}\right) \operatorname{dim}_{q}\left(\Lambda_{\pi}\right) q^{\operatorname{deg}\left(D_{\pi}\right)}\left(\sum_{b \in \mathfrak{B}_{\pi}} q^{\operatorname{deg}(b)}\right) \\
& \leq \sum_{\pi \in \Pi(\alpha)}\left(\sum_{b \in \mathfrak{B}_{\pi}} q^{\operatorname{deg}(b v-)}\right)^{2} l_{\pi} \\
& =\sum_{\pi \in \Pi(\alpha)} \operatorname{dim}_{q}(\bar{\Delta}(\pi))^{2} l_{\pi}=\operatorname{dim}_{q}\left(R_{\alpha}\right),
\end{aligned}
$$

by Corollary 2.16. The inequalities are therefore equalities, and this implies that the spanning set $\left\{b e_{\pi} f D_{\pi}\left(b^{\prime}\right)^{\tau} \mid \pi \in \Pi(\alpha), b, b^{\prime} \in \mathfrak{B}_{\pi}, f \in X_{\pi}\right\}$ of $R_{\alpha}$ is a basis and $\operatorname{dim}_{q} \Lambda_{\pi}=l_{\pi}$ for all $\pi$.

To show (i) and (ii), we have already noted that the claimed bases span $\Delta(\beta)$ and $\Delta^{\prime}(\beta)$, respectively. We now apply part (iii) to see that they are linearly independent.

Corollary 3.28. The set $\left\{b e_{\pi} f D_{\pi}\left(b^{\prime}\right)^{\tau} \mid \pi \in \Pi(\alpha), b, b^{\prime} \in \mathfrak{B}_{\pi}, f \in X_{\pi}\right\}$ is an $\mathcal{O}$-basis for $R_{\alpha}$.

Proof. Apply Proposition 3.27(iii) and the fact that the filtration by the ideals $I_{\pi}$ exhausts $R_{\alpha}$, which follows from Lemma 3.1 . 


\section{Affine Cellularity}

Recall the notion of an affine cellular algebra from the introduction. In this section, we fix $\alpha \in Q_{+}$and prove that $R_{\alpha}$ is affine cellular over $\mathbb{Z}$ (which then implies that it is affine cellular over any $k$ ).

For any $\pi \in \Pi(\alpha)$, we define

$$
I_{\pi}^{\prime}:=\mathbb{Z}-\operatorname{span}\left\{b e_{\pi} \Lambda_{\pi} D_{\pi}\left(b^{\prime}\right)^{\tau} \mid b, b^{\prime} \in \mathfrak{B}_{\pi}\right\} .
$$

By Corollary 3.28 , we have $R_{\alpha}=\oplus_{\pi \in \Pi(\alpha)} I_{\pi}^{\prime}$. Moreover, $\tau\left(I_{\pi}^{\prime}\right)=I_{\pi}^{\prime}$. Indeed, $\delta_{\pi}$ commutes with elements of $\Lambda_{\pi}$ in view of Hypothesis [3.9(iv). So by Lemma 3.12, we have

$$
\begin{aligned}
\tau\left(I_{\pi}^{\prime}\right) & =\mathbb{Z}-\operatorname{span}\left\{b^{\prime} D_{\pi}^{\tau} \Lambda_{\pi}^{\tau} \delta_{\pi}^{\tau} D_{\pi}^{\tau} b^{\tau} \mid b, b^{\prime} \in \mathfrak{B}_{\pi}\right\} \\
& =\mathbb{Z}-\operatorname{span}\left\{b^{\prime} D_{\pi} \delta_{\pi} \Lambda_{\pi} D_{\pi} b^{\tau} \mid b, b^{\prime} \in \mathfrak{B}_{\pi}\right\}=I_{\pi}^{\prime} .
\end{aligned}
$$

By Proposition 3.27, we have $I_{\pi}=\oplus_{\sigma \geq \pi} I_{\sigma}^{\prime}$, and we have a nested family of ideals $\left(I_{\pi}\right)_{\pi \in \Pi(\alpha)}$. To check that $R_{\alpha}$ is affine cellular, we need to verify that $\bar{I}_{\pi}:=I_{\pi} / I_{>\pi}$ is an affine cell ideal in $\bar{R}_{\alpha}:=R_{\alpha} / I_{>\pi}$. As usual we denote $\bar{x}:=x+I_{>\pi} \in \bar{R}_{\alpha}$ for $x \in R_{\alpha}$.

The affine algebra $B$ in the definition of a cell ideal will be the algebra $\Lambda_{\pi}$, with the automorphism $\sigma$ being the identity map. The $\mathbb{Z}$-module $V$ will be the formal free $\mathbb{Z}$-module $V_{\pi}$ on the basis $\mathfrak{B}_{\pi}$. By Corollary [3.26(i) and Proposition 3.27, the following maps are isomomorphisms of $\Lambda_{\pi}$-modules.

$$
\begin{aligned}
& \eta_{\pi}: V_{\pi} \otimes_{\mathbb{Z}} \Lambda_{\pi} \rightarrow \Delta(\pi), b \otimes f \mapsto \bar{b} \bar{f} \bar{e}_{\pi}, \\
& \eta_{\pi}^{\prime}: \Lambda_{\pi} \otimes_{\mathbb{Z}} V_{\pi} \rightarrow \Delta^{\prime}(\pi), f \otimes b \mapsto \bar{e}_{\pi} \bar{f} \bar{D}_{\pi} \bar{b}^{\tau} .
\end{aligned}
$$

This allows us to endow $V_{\pi} \otimes_{\mathbb{Z}} \Lambda_{\pi}$ with a structure of an $\left(R_{\alpha}, \Lambda_{\pi}\right)$-bimodule and $\Lambda_{\pi} \otimes_{\mathbb{Z}} V_{\pi}$ with a structure of an $\left(\Lambda_{\pi}, R_{\alpha}\right)$-bimodule.

In view of Corollary 3.26)(iii), (iv) we see that $\Delta(\pi)$ (resp. $\Delta^{\prime}(\pi)$ ) is a right (resp. left) $\Lambda_{\pi}$-module, and so we may define an $R_{\alpha}$-bimodule homomorphism

$$
\nu_{\pi}: \Delta(\pi) \otimes_{\Lambda_{\pi}} \Delta^{\prime}(\pi) \rightarrow I_{\pi} / I_{>\pi}, \bar{r} \bar{e}_{\pi} \otimes \bar{e}_{\pi} \bar{r}^{\prime} \mapsto \bar{r} \bar{e}_{\pi} \bar{r}^{\prime} .
$$

By Proposition 3.27, $\nu_{\pi}$ is an isomorphism. Let $\mu_{\pi}:=\nu_{\pi}^{-1}$. This will be the map $\mu$ in the definition of a cell ideal.

Theorem 4.1. The above data make $R_{\alpha}$ into an affine cellular algebra.

Proof. To verify that $\bar{I}_{\pi}$ is a cell ideal in $\bar{R}_{\alpha}$, we first check that our $\left(\Lambda_{\pi}, R_{\alpha}\right)$-bimodule structure on $\Lambda_{\pi} \otimes_{\mathbb{Z}} V_{\pi}$ comes from our $\left(R_{\alpha}, \Lambda_{\pi}\right)$-bimodule structure on $V_{\pi} \otimes_{\mathbb{Z}} \Lambda_{\pi}$ via the rule (1.1). Let $\mathrm{s}_{\pi}: V_{\pi} \otimes_{\mathbb{Z}} \Lambda_{\pi} \stackrel{\sim}{\longrightarrow} \Lambda_{\pi} \otimes_{\mathbb{Z}} V_{\pi}$ be the swap map. This is equivalent to the fact that the composition map

$$
\varphi: \Delta^{\prime}(\pi) \stackrel{\left(\eta_{\pi}^{\prime}\right)^{-1}}{\longrightarrow} \Lambda_{\pi} \otimes_{\mathbb{Z}} V_{\pi} \stackrel{\mathrm{s}_{\pi}^{-1}}{\rightarrow} V_{\pi} \otimes_{\mathbb{Z}} \Lambda_{\pi} \stackrel{\eta_{\pi}}{\rightarrow} \Delta(\pi)=\Delta(\pi)^{\tau},
$$

is an isomorphism of right $R_{\alpha}$-modules. We already know that this is an isomorphism of $\mathbb{Z}$-modules, and so it suffices to check that

$$
\varphi\left(\bar{e}_{\pi} \bar{f} \bar{D}_{\pi} \bar{c}^{\tau} \bar{r}\right)=\bar{r}^{\tau} \varphi\left(\bar{e}_{\pi} \bar{f} \bar{D}_{\pi} \bar{c}^{\tau}\right)
$$


for all $f \in \Lambda_{\pi}, c \in \mathfrak{B}_{\pi}$, and $r \in R_{\alpha}$. Note that $\varphi\left(\bar{e}_{\pi} \bar{f} \bar{D}_{\pi} \bar{c}^{\tau}\right)=\bar{c} \bar{f} \bar{e}_{\pi}$. So we have to check

$$
\varphi\left(\bar{e}_{\pi} \bar{f} \bar{D}_{\pi} \bar{c}^{\tau} \bar{r}\right)=\bar{r}^{\tau} \bar{c} \bar{f} \bar{e}_{\pi} .
$$

By Proposition 3.27 (ii) we can find $\left\{f_{b} \mid b \in \mathfrak{B}_{\pi}\right\} \subseteq \Lambda_{\pi}$ such that

$$
\bar{e}_{\pi} \bar{f} \bar{D}_{\pi} \bar{c}^{\tau} \bar{r}=\sum_{b \in \mathfrak{B}_{\pi}} \bar{e}_{\pi} \bar{f}_{b} \bar{D}_{\pi} \bar{b}^{\tau}
$$

Also, by Corollary 3.26(i), we have

$$
\bar{e}_{\pi} \bar{f} \bar{D}_{\pi} \bar{c}^{\tau}=\bar{f} \bar{e}_{\pi} \bar{D}_{\pi} \bar{c}^{\tau}=\bar{e}_{\pi} \bar{D}_{\pi} \bar{f} \bar{c}^{\tau}
$$

Using this and the $\tau$-invariance of $D_{\pi}$ and $\delta_{\pi}$, we get (4.2) as follows:

$$
\begin{aligned}
\bar{r}^{\tau} \bar{c} \bar{f}_{\bar{e}} & =\bar{r}^{\tau} \bar{c} \bar{e}_{\pi}^{2}=\bar{r}^{\tau} \bar{c} \bar{f} \bar{D}_{\pi}^{\tau} \bar{\delta}_{\pi}^{\tau} \bar{D}_{\pi}^{\tau} \bar{\delta}_{\pi}^{\tau}=\left(\bar{\delta}_{\pi} \bar{D}_{\pi} \bar{\delta}_{\pi} \bar{D}_{\pi} \bar{f} \bar{c}^{\tau} \bar{r}\right)^{\tau} \\
& =\left(\bar{\delta}_{\pi} \bar{e}_{\pi} \bar{D}_{\pi} \bar{f} \bar{c}^{\tau} \bar{r}\right)^{\tau}=\left(\bar{\delta}_{\pi} \bar{e}_{\pi} \bar{f} \bar{D}_{\pi} \bar{c}^{\tau} \bar{r}\right)^{\tau}=\left(\bar{\delta}_{\pi} \sum_{b \in \mathfrak{B}_{\pi}} \bar{e}_{\pi} \bar{f}_{b} \bar{D}_{\pi} \bar{b}^{\tau}\right)^{\tau} \\
& =\left(\sum_{b \in \mathfrak{B}_{\pi}} \bar{\delta}_{\pi} \bar{D}_{\pi} \bar{\delta}_{\pi} \bar{D}_{\pi} \bar{f}_{b} \bar{b}^{\tau}\right)^{\tau}=\sum_{b \in \mathfrak{B}_{\pi}} \bar{b} \bar{f}_{b} \bar{D}_{\pi} \bar{\delta}_{\pi} \bar{D}_{\pi} \bar{\delta}_{\pi}=\sum_{b \in \mathfrak{B}_{\pi}} \bar{b} \bar{f}_{b} \bar{e}_{\pi}^{2} \\
& =\sum_{b \in \mathfrak{B}_{\pi}} \bar{b} \bar{f}_{b} \bar{e}_{\pi},
\end{aligned}
$$

which equals the left hand side of (4.2) by definition of $\varphi$.

To complete the proof, it remains to verify the commutativity of (1.2). This is equivalent to

$$
\tau \circ \nu_{\pi} \circ\left(\eta_{\pi} \otimes \eta_{\pi}^{\prime}\right)\left((b \otimes f) \otimes\left(f^{\prime} \otimes b^{\prime}\right)\right)=\nu_{\pi} \circ\left(\eta_{\pi} \otimes \eta_{\pi}^{\prime}\right)\left(\left(b^{\prime} \otimes f^{\prime}\right) \otimes(f \otimes b)\right)
$$

for all $b, b^{\prime} \in \mathfrak{B}_{\pi}$ and $f, f^{\prime} \in \Lambda_{\pi}$. The left hand side equals

$$
\begin{aligned}
& \tau \circ \nu_{\pi}\left(\bar{b} \bar{f} \bar{e}_{\pi} \otimes \bar{e}_{\pi} \bar{f}^{\prime} \bar{D}_{\pi}\left(\bar{b}^{\prime}\right)^{\tau}\right)=\tau\left(\bar{b} \bar{f} \bar{e}_{\pi} \bar{f}^{\prime} \bar{D}_{\pi}\left(\bar{b}^{\prime}\right)^{\tau}\right)=\tau\left(\bar{b} \bar{e}_{\pi} \bar{f} \bar{f}^{\prime} \bar{D}_{\pi}\left(\bar{b}^{\prime}\right)^{\tau}\right) \\
& =\bar{b}^{\prime} \bar{D}_{\pi} \bar{f}^{\prime} \bar{f} \bar{e}_{\pi}^{\tau} \bar{b}^{\tau}=\bar{b}^{\prime} \bar{D}_{\pi} \bar{f}^{\prime} \bar{f} \bar{\delta}_{\pi} \bar{D}_{\pi} \bar{b}^{\tau}=\bar{b}^{\prime} \bar{D}_{\pi} \delta_{\pi} \bar{f}^{\prime} \bar{f}^{\bar{f}} D_{\pi} \bar{b}^{\tau}=\bar{b}^{\prime} \bar{e}_{\pi} \bar{f}^{\prime} \bar{f} \bar{D}_{\pi} \bar{b}^{\tau} \\
& =\bar{b}^{\prime} \bar{f}^{\prime} \bar{e}_{\pi} \bar{f} \bar{D}_{\pi} \bar{b}^{\tau}=\nu_{\pi}\left(\bar{b}^{\prime} \bar{f}^{\prime} \bar{e}_{\pi} \otimes \bar{e}_{\pi} \bar{f} \bar{D}_{\pi} \bar{b}^{\tau}\right),
\end{aligned}
$$

which equals $\nu_{\pi} \circ\left(\eta_{\pi} \otimes \eta_{\pi}^{\prime}\right)\left(\left(b^{\prime} \otimes f^{\prime}\right) \otimes(f \otimes b)\right)$, as required.

\section{Verification of the Hypothesis}

In this section we verify Hypothesis 3.9 for all finite types. In ADE types (with one exception) this can be do using the theory of homogeneous representations developed in 12 . This theory is reviewed in the next subsection. We use the cuspidal modules of 4 .

Throughout the section $\beta$ is a positive root, and $\bar{R}_{\beta}:=R_{\beta} / I_{>(\beta)}, \bar{r}:=$ $r+I_{>(\beta)}$ for $r \in R_{\beta}$. 
5.1. Homogeneous representations. In this section we assume that the Cartan matrix $A$ is symmetric. In this subsection we fix $\alpha \in Q_{+}$with $d=\operatorname{ht}(\alpha)$. A graded $R_{\alpha}$-module is called homogeneous if it is concentrated in one degree. Let $\boldsymbol{i} \in\langle I\rangle_{\alpha}$. We call $s_{r} \in S_{d}$ an admissible transposition for $\boldsymbol{i}$ if $a_{i_{r}, i_{r+1}}=0$. The word graph $G_{\alpha}$ is the graph with the set of vertices $\langle I\rangle_{\alpha}$, and with $\boldsymbol{i}, \boldsymbol{j} \in\langle I\rangle_{\alpha}$ connected by an edge if and only if $\boldsymbol{j}=s_{r} \boldsymbol{i}$ for some admissible transposition $s_{r}$ for $\boldsymbol{i}$. A connected component $C$ of $G_{\alpha}$ is called homogeneous if for some $\boldsymbol{i}=\left(i_{1}, \ldots, i_{d}\right) \in C$ the following condition holds:

if $i_{r}=i_{s}$ for some $r<s$ then there exist $t, u$ such that $r<t<u<s$ and $a_{i_{r}, i_{t}}=a_{i_{r}, i_{u}}=-1$.

Theorem 5.1. [12, Theorems 3.6, 3.10, (3.3)] Let $C$ be a homogeneous connected component of $G_{\alpha}$. Let $L(C)$ be the vector space concentrated in degree 0 with basis $\left\{v_{\boldsymbol{i}} \mid \boldsymbol{i} \in C\right\}$ labeled by the elements of $C$. The formulas

$$
\begin{aligned}
1_{\boldsymbol{j}} v_{\boldsymbol{i}} & =\delta_{\boldsymbol{i}, \boldsymbol{j}} v_{\boldsymbol{i}} \quad\left(\boldsymbol{j} \in\langle I\rangle_{\alpha}, \boldsymbol{i} \in C\right), \quad(1 \leq r \leq d, \boldsymbol{i} \in C), \\
y_{r} v_{\boldsymbol{i}} & =0 \quad(1 \leq r<d, \boldsymbol{i} \in C) \\
\psi_{r} v_{\boldsymbol{i}} & = \begin{cases}v_{s_{r}} \boldsymbol{i} & \text { if } s_{r} \boldsymbol{i} \in C, \\
0 & \text { otherwise } ;\end{cases}
\end{aligned}
$$

define an action of $R_{\alpha}$ on $L(C)$, under which $L(C)$ is a homogeneous irreducible $R_{\alpha}$-module. Furthermore, $L(C) \neq L\left(C^{\prime}\right)$ if $C \neq C^{\prime}$, and every homogeneous irreducible $R_{\alpha}$-module, up to a degree shift, is isomorphic to one of the modules $L(C)$.

We need to push the theory of homogeneous modules a little further. In Proposition 5.3 below we give a presentation for a homogeneous module as a cyclic modules generated by a word vector. Let $C$ be a homogeneous component of $G_{\alpha}$ and $\boldsymbol{i} \in C$. An element $w \in \mathfrak{S}_{d}$ is called $\boldsymbol{i}$-admissible if it can be written as $w=s_{r_{1}} \ldots s_{r_{b}}$, where $s_{r_{a}}$ is an admissible transposition for $s_{r_{a+1}} \ldots s_{r_{b}} \boldsymbol{i}$ for all $a=1, \ldots, b$. We denote the set of all $\boldsymbol{i}$-admissible elements by $\mathfrak{D}_{\boldsymbol{i}}$.

Lemma 5.2. Let $C$ be a homogeneous component of $G_{\alpha}$ and $\boldsymbol{i} \in C$. Then $\left\{\psi_{w} v_{\boldsymbol{i}} \mid w \in \mathfrak{D}_{\boldsymbol{i}}\right\}$ is a basis of $L(C)$.

Proof. $\quad$ Note that if $w, w^{\prime}$ are admissible elements, then $w=w^{\prime}$ if and only if $w \boldsymbol{i}=w^{\prime} \boldsymbol{i}$. Indeed, it suffices to prove that $w \boldsymbol{i}=\boldsymbol{i}$ implies $w=1$, which follows from the property (5.1). The lemma follows.

Proposition 5.3. Let $C$ be a homogeneous component of $G_{\alpha}$ and $\boldsymbol{i} \in C$. Let $J(\boldsymbol{i})$ be the left ideal of $R_{\alpha}$ generated by

$$
\left\{y_{r}, 1_{j}, \psi_{w} 1_{\boldsymbol{i}} \mid 1 \leq r \leq d, \boldsymbol{j} \in\langle I\rangle_{\alpha} \backslash \boldsymbol{i}, w \in \mathfrak{S}_{d} \backslash \mathfrak{D}_{\boldsymbol{i}}\right\} .
$$

Then $R_{\alpha} / J_{\alpha} \simeq L(C)$ as (graded) left $R_{\alpha}$-modules. 
Proof. Note that the elements in (5.2) annihilate the vector $v_{\boldsymbol{i}} \in L(C)$, which generates $L(C)$, whence we have a (homogeneous) surjection

$$
R_{\alpha} / J_{\alpha} \rightarrow L(C), h+J_{\alpha} \mapsto h v_{i}
$$

To prove that this surjection is an isomorphism it suffices to prove that the dimension of $R_{\alpha} / J_{\alpha}$ is at most $\operatorname{dim} L(C)=|C|$, which follows easily from Lemma 5.2

5.2. Special Lyndon orders. Recall the theory of standard modules reviewed in 2.5. We now specialize to the case of a Lyndon convex order on $\Phi_{+}$as studied in [11]. For this we first need to fix a total order ' $\leq$' on $I$. This gives rise to a lexicographic order ' $\leq$ ' on the set $\langle I\rangle$. In particular, each finite dimensional $R_{\alpha}$-module has its (lexicographically) highest word, and the highest word of an irreducible module determines the irreducible module uniquely up to an isomorphism. This leads to the natural notion of dominant words (called good words in [11]), namely the elements of $\langle I\rangle_{\alpha}$ which occur as highest words of finite dimensional $R_{\alpha}$-modules.

The dominant words of cuspidal modules are characterized among all dominant words by the property that they are Lyndon words, so we refer to them as dominant Lyndon words. There is an explicit bijection

$$
\Phi_{+} \rightarrow\{\text { dominant Lyndon words }\}, \beta \mapsto \boldsymbol{i}_{\beta},
$$

uniquely determined by the property $\left|\boldsymbol{i}_{\beta}\right|=\beta$. Note that this notation $\boldsymbol{i}_{\beta}$ will be consistent with the same notation used in 33.1 .

Setting $\beta \leq \gamma$ if and only if $\boldsymbol{i}_{\beta} \leq \boldsymbol{i}_{\gamma}$ for $\beta, \gamma \in \Phi_{+}$defines a total order on $\Phi_{+}$called a Lyndon order. It is known that each Lyndon order is convex, and the theory of standard modules for Lyndon orders, developed in [11], fits into the general theory described in 2.5 . However, working with Lyndon orders allows us to be a little more explicit. In particular, given a a root partition $\pi=\left(p_{1}, \ldots, p_{N}\right) \in \Pi(\alpha)$, set

$$
\boldsymbol{i}_{\pi}:=\boldsymbol{i}_{\beta_{1}}^{p_{1}} \ldots \boldsymbol{i}_{\beta_{N}}^{p_{N}} \in\langle I\rangle_{\alpha} .
$$

Lemma 5.4. [11, Theorem 7.2] Let $\pi \in \Pi(\alpha)$. Then $\boldsymbol{i}_{\pi}$ is the highest word of $L(\pi)$.

From now on, we fix the notation for the Dynkin diagrams as follows:

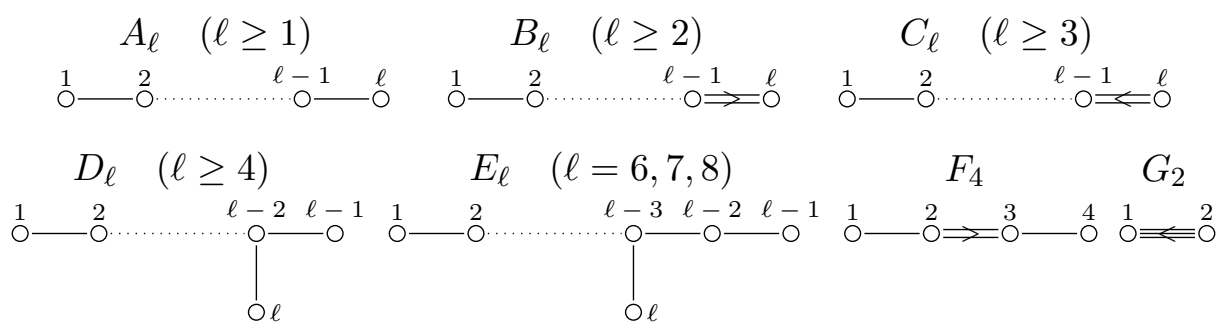

Also, we choose the signs $\varepsilon_{i j}$ as in $\$ 2.3$ and the total order $\leq$ on $I$ so that $\varepsilon_{i j}=1$ and $i<j$ if the corresponding labels $i$ and $j$ satisfy $i<j$ as integers. 
5.3. Homogeneous roots. We stick with the choices made in 55.2 , Throughout the subsection, we assume that the Cartan matrix is of $A D E$ type and $\beta \in \Phi_{+}$is such that $\boldsymbol{i}_{\beta}$ is homogeneous. Let $d:=\operatorname{ht}(\beta)$. The module $L(\beta)$ is concentrated in degree 0 , and each of its word spaces is one dimensional. Set $\mathfrak{D}_{\beta}:=\mathfrak{D}_{\boldsymbol{i}_{\beta}}$. Then we can take $\mathfrak{B}_{\beta}=\left\{\psi_{w} e\left(\boldsymbol{i}_{\beta}\right) \mid w \in \mathfrak{D}_{\beta}\right\}$. Let $\delta_{\beta}=D_{\beta}=e\left(\boldsymbol{i}_{\beta}\right)$, and define $y_{\beta}:=y_{d} e\left(\boldsymbol{i}_{\beta}\right)$. All parts of Hypothesis 3.9 are trivially satisfied, except $(\mathrm{v})$. In the rest of this subsection we verify Hypothesis 3.9(v).

Lemma 5.5. Let $w \in \mathfrak{S}_{d} \backslash \mathfrak{D}_{\beta}$. Then $\psi_{w} P_{d} e\left(\boldsymbol{i}_{\beta}\right) \subseteq I_{>(\beta)}$.

Proof. We have $\psi_{w}=\psi_{r_{1}} \ldots \psi_{r_{m}}$ for a reduced decomposition $w=$ $s_{r_{1}} \ldots s_{r_{m}}$. Let $k$ be the largest index such that $s_{r_{k}}$ is not an admissible transposition of $s_{r_{k+1}} \ldots s_{r_{m}} \boldsymbol{i}_{\beta}$. By Theorem 5.1, $s_{r_{k}} \ldots s_{r_{m}} \boldsymbol{i}_{\beta}$ is not a word of $L(\beta)$. So by Corollary 3.3 ,

$$
\psi_{r_{k}} \ldots \psi_{r_{m}} P_{d} e\left(\boldsymbol{i}_{\beta}\right)=e\left(s_{r_{k}} \ldots s_{r_{m}} \boldsymbol{i}_{\beta}\right) \psi_{r_{k}} \ldots \psi_{r_{m}} P_{d} e\left(\boldsymbol{i}_{\beta}\right) \subseteq I_{>(\beta)},
$$

whence $\psi_{w} P_{d} e\left(\boldsymbol{i}_{\beta}\right) \subseteq I_{>(\beta)}$.

Lemma 5.6. Given $1 \leq r, s \leq d$, we have $\left(y_{s}-y_{r}\right) e\left(\boldsymbol{i}_{\beta}\right) \in I_{>(\beta)}$.

Proof. We prove by induction on $s=1, \ldots, d$ that $\left(y_{s}-y_{r}\right) e\left(\boldsymbol{i}_{\beta}\right) \in I_{>(\beta)}$ for all $1 \leq r \leq s$. The base case $s=1$ is trivial. Let $s>1$, and write $\boldsymbol{i}_{\beta}=\left(i_{1}, \ldots, i_{d}\right)$. If $i_{r} \cdot i_{s}=0$ for all $1 \leq r<s$, then

$$
\left(i_{s}, i_{1}, i_{2}, \ldots, i_{s-1}, i_{s+1}, \ldots, i_{d}\right)
$$

is a word of $L(\beta)$. On the other hand, Lemma 5.4 says that $\boldsymbol{i}_{\beta}$ is the largest word of $L(\beta)$ and so $i_{s}<i_{1}$. But then $\boldsymbol{i}_{\beta}$ is not a Lyndon word, which is a contradiction. Thus there exists some $r<s$ with $i_{r} \cdot i_{s} \neq 0$. Since the Cartan matrix is assumed to be of ADE type, either $i_{r} \cdot i_{s}=-1$ or $i_{r}=i_{s}$. In the second case, by homogeneity (5.1) we can find $r<r^{\prime}<s$ with $i_{r^{\prime}} \cdot i_{s}=-1$. This shows that the definition $t:=\max \left\{r \mid r<s\right.$ and $\left.i_{r} \cdot i_{s}=-1\right\}$ makes sense. Once again by homogeneity we must have that $i_{r} \cdot i_{s}=0$ for any $r$ with $t<r<s$. Therefore, using defining relations in $R_{\alpha}$, we get

$$
\left(\psi_{s-1} \ldots \psi_{t}\right)\left(\psi_{t} \ldots \psi_{s-1}\right) e\left(\boldsymbol{i}_{\beta}\right)= \pm\left(y_{s}-y_{t}\right) e\left(\boldsymbol{i}_{\beta}\right) .
$$

On the other hand, the cycle $(t, t+1, \ldots, s)$ is not an element of $\mathfrak{D}_{\beta}$. By Lemma 5.5 we must have $\psi_{t} \ldots \psi_{s-1} e\left(\boldsymbol{i}_{\beta}\right) \in I_{>(\beta)}$. This shows that $\left(y_{s}-\right.$ $\left.y_{t}\right) e\left(\boldsymbol{i}_{\beta}\right) \in I_{>(\beta)}$, and therefore by induction that $\left(y_{s}-y_{r}\right) e\left(\boldsymbol{i}_{\beta}\right) \in I_{>(\beta)}$ for every $r$ with $1 \leq r \leq s$.

Recall the notation $\bar{R}_{\beta}:=R_{\beta} / I_{>(\beta)}$ and $\bar{r}:=r+I_{>(\beta)} \in \bar{R}_{\beta}$ for $r \in R_{\beta}$.

Corollary 5.7. We have that $\bar{e}_{\beta} \bar{R}_{\beta} \bar{e}_{\beta}$ is generated by $\bar{y}_{\beta}$.

Proof. By Theorem 2.2, an element of $e_{\beta} R_{\beta} e_{\beta}$ is a linear combination of terms of the form $\psi_{w} y_{1}^{a_{1}} \ldots y_{d}^{a_{d}} e\left(\boldsymbol{i}_{\beta}\right)$ such that $w \boldsymbol{i}_{\beta}=\boldsymbol{i}_{\beta}$. If $w \notin \mathfrak{D}_{\beta}$, then $\psi_{w} e_{\beta} \in I_{>(\beta)}$ by Lemma [5.5]. Otherwise, Lemma [5.2 shows that $w=1$. 
Therefore, $\bar{e}_{\beta} \bar{R}_{\beta} \bar{e}_{\beta}$ is spanned by terms of the form $\bar{y}_{1}^{a_{1}} \ldots \bar{y}_{d}^{a_{d}} \bar{e}_{\beta}$. In view of Lemma 5.6. we see that $\bar{e}_{\beta} \bar{R}_{\beta} \bar{e}_{\beta}$ is generated by $\bar{y}_{\beta}=\bar{y}_{d}$.

5.4. Types $A D E$. Throughout the subsection, we assume again that the Cartan matrix is of $A D E$ type. By [4], with a correction made in [2, Lemma A7], if $\beta \in \Phi_{+}$is any positive root, except the highest root in type $E_{8}$, then $\boldsymbol{i}_{\beta}$ is homogeneous. We have proved in the previous subsection that Hypothesis 3.9 holds in this case.

Now, we deal with the highest root

$$
\theta:=2 \alpha_{1}+3 \alpha_{2}+4 \alpha_{3}+5 \alpha_{4}+6 \alpha_{5}+4 \alpha_{6}+2 \alpha_{7}+3 \alpha_{8}
$$

in type $E_{8}$. By [2, Example A.5], the corresponding Lyndon word is

$$
\boldsymbol{i}_{\theta}=12345867564534231234586756458 .
$$

Define the positive roots

$$
\begin{aligned}
& \theta_{1}:=\alpha_{1}+\alpha_{2}+\alpha_{3}+2 \alpha_{4}+3 \alpha_{5}+2 \alpha_{6}+\alpha_{7}+2 \alpha_{8}, \\
& \theta_{2}:=\alpha_{1}+2 \alpha_{2}+3 \alpha_{3}+3 \alpha_{4}+3 \alpha_{5}+2 \alpha_{6}+\alpha_{7}+\alpha_{8} .
\end{aligned}
$$

Then the root partition $\left(\theta_{1}, \theta_{2}\right)$ is a minimal element of $\Pi(\theta) \backslash\{(\theta)\}$. Moreover, $\boldsymbol{i}_{\theta_{2}}=1234586756453423$ and $\boldsymbol{i}_{\theta_{1}}=1234586756458$. Indeed, one sees by inspection that these words are highest words in the corresponding homogeneous representations and are Lyndon. Finally, we have $\boldsymbol{i}_{\theta}=\boldsymbol{i}_{\theta_{2}} \boldsymbol{i}_{\theta_{1}}$.

Denote by $v_{\theta_{1}}$ and $v_{\theta_{2}}$ non-zero vectors in the $\boldsymbol{i}_{\theta_{1}}$ - and $\boldsymbol{i}_{\theta_{2}}$-word spaces in the homogeneous modules $L\left(\theta_{1}\right)$ and $L\left(\theta_{2}\right)$, respectively. Note that $L\left(\theta_{1}\right) \otimes L\left(\theta_{2}\right)$ is naturaly a submodule of $L\left(\theta_{1}\right) \circ L\left(\theta_{2}\right)$, so we can consider $v_{\theta_{1}} \otimes v_{\theta_{2}}$ as a cyclic vector of $L\left(\theta_{1}\right) \circ L\left(\theta_{2}\right)$, and similarly $v_{\theta_{2}} \otimes v_{\theta_{1}}$ as a cyclic vector of $L\left(\theta_{2}\right) \circ L\left(\theta_{1}\right)$. By definition, $L\left(\theta_{1}\right) \circ L\left(\theta_{2}\right)$ is the proper standard module $\bar{\Delta}\left(\theta_{1}, \theta_{2}\right)$, and let $v_{\theta_{1}, \theta_{2}}$ be the image of $v_{\theta_{1}} \otimes v_{\theta_{2}}$ under the natural projection $\bar{\Delta}\left(\theta_{1}, \theta_{2}\right) \rightarrow L\left(\theta_{1}, \theta_{2}\right)$. Denote by $w(\theta)$ the element of $\mathfrak{S}_{29}$ which sends $(1, \ldots, 29)$ to $(17, \ldots, 29,1, \ldots, 16)$. The following has been established in [2], see especially [2, Theorem A.9, Proof], but we sketch its very easy proof for the reader's convenience.

Lemma 5.8. The multiplicity of the highest word $\boldsymbol{i}_{\theta}$ in $L(\theta)$ is one. Moreover, there is a non-zero vector $v_{\theta}$ in the $\theta$-word space of $L(\theta)$ and homogeneous $R_{\theta}$-module maps

$$
\begin{aligned}
& \mu: L\left(\theta_{1}, \theta_{2}\right)\langle 1\rangle \rightarrow L\left(\theta_{2}\right) \circ L\left(\theta_{1}\right), v_{\theta_{1}, \theta_{2}} \mapsto \psi_{w(\theta)}\left(v_{\theta_{2}} \otimes v_{\theta_{1}}\right), \\
& \nu: L\left(\theta_{2}\right) \circ L\left(\theta_{1}\right) \rightarrow L(\theta), v_{\theta_{2}} \otimes v_{\theta_{1}} \mapsto v_{\theta},
\end{aligned}
$$

such that the sequence

$$
0 \rightarrow L\left(\theta_{1}, \theta_{2}\right)\langle 1\rangle \stackrel{\mu}{\rightarrow} L\left(\theta_{2}\right) \circ L\left(\theta_{1}\right) \stackrel{\nu}{\rightarrow} L(\theta) \rightarrow 0
$$

is exact. Finally,

$$
\operatorname{ch}_{q} L(\theta)=\left(\operatorname{ch}_{q} L\left(\theta_{2}\right) \circ \operatorname{ch}_{q} L\left(\theta_{1}\right)-q \operatorname{ch}_{q} L\left(\theta_{1}\right) \circ \operatorname{ch}_{q} L\left(\theta_{2}\right)\right) /\left(1-q^{2}\right) .
$$


Proof. By [11, Theorem 7.2(ii)], the multiplicity of the word $\boldsymbol{i}_{\theta_{1}} \boldsymbol{i}_{\theta_{2}}$ in $L\left(\theta_{1}\right) \circ L\left(\theta_{2}\right)$ is 1 . Moreover, an explicit check shows that the multiplicity of $\boldsymbol{i}_{\theta}$ in $L\left(\theta_{1}\right) \circ L\left(\theta_{2}\right)$ is $q$. We conclude using Theorem 2.6 and the minimality of $\left(\theta_{1}, \theta_{2}\right)$ in $\Pi(\theta) \backslash\{(\theta)\}$ that the standard module $L\left(\theta_{1}\right) \circ L\left(\theta_{2}\right)$ is uniserial with head $L\left(\theta_{1}, \theta_{2}\right)$ and socle $L(\theta)\langle 1\rangle$. The result follows from these observations since $L\left(\theta_{1}, \theta_{2}\right)$ is $\circledast$-self-dual and $\left(L\left(\theta_{1}\right) \circ L\left(\theta_{2}\right)\right)^{\circledast} \simeq L\left(\theta_{2}\right) \circ L\left(\theta_{1}\right)\langle-1\rangle$ in view of [15, Theorem 2.2].

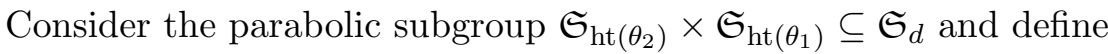

$$
\mathfrak{D}_{\theta_{2}, \theta_{1}}:=\left\{\left(w_{2}, w_{1}\right) \in \mathfrak{S}_{\mathrm{ht}\left(\theta_{2}\right)} \times \mathfrak{S}_{\mathrm{ht}\left(\theta_{1}\right)} \mid w_{2} \in \mathfrak{D}_{\theta_{2}}, w_{1} \in \mathfrak{D}_{\theta_{1}}\right\} .
$$

With this notation we finally have:

Lemma 5.9. The cuspidal module $L(\theta)$ is generated by a degree 0 vector $v_{\theta}$ subject only to the relations:

$$
\begin{aligned}
\left(e(\boldsymbol{j})-\delta_{\boldsymbol{j}, \boldsymbol{i}_{\theta}}\right) v_{\theta}=0, & \text { for all } \boldsymbol{j} \in\langle I\rangle_{\theta}, \\
y_{r} v_{\theta}=0, & \text { for all } r=1, \ldots, \mathrm{ht}(\theta), \\
\psi_{w} v_{\theta}=0, & \text { for all } w \in\left(\mathfrak{S}_{\mathrm{ht}\left(\theta_{2}\right)} \times \mathfrak{S}_{\mathrm{ht}\left(\theta_{1}\right)}\right) \backslash \mathfrak{D}_{\theta_{2}, \theta_{1}}, \\
\psi_{w(\theta)} v_{\theta}=0 . &
\end{aligned}
$$

Proof. The theorem follows easily from Proposition 5.3 applied to homogeneous modules $L\left(\theta_{1}\right)$ and $L\left(\theta_{2}\right)$, and Lemma 5.8 .

We now define $\delta_{\theta}=D_{\theta}=e\left(\boldsymbol{i}_{\theta}\right)$, and $y_{\theta}=y_{\mathrm{ht}(\theta)} e\left(\boldsymbol{i}_{\theta}\right)$. All parts of Hypothesis 3.9 are trivially satisfied, except (v). We now verify Hypothesis 3.9(v).

Lemma 5.10. We have

$$
\iota_{\theta_{2}, \theta_{1}}\left(I_{>\left(\theta_{2}\right)} \otimes R_{\theta_{1}}+R_{\theta_{2}} \otimes I_{>\left(\theta_{1}\right)}\right) \subseteq I_{>(\theta)} .
$$

Proof. Apply Proposition 3.5 twice with $m=2, \gamma_{1}=\theta_{2}, \gamma_{2}=\theta_{1}, \pi=(\theta)$, and either $k=1$ and $\pi_{0}=\left(\theta_{2}\right)$, or $k=2$ and $\pi_{0}=\left(\theta_{1}\right)$.

Lemma 5.11. We have that $\bar{e}_{\theta} \bar{R}_{\theta} \bar{e}_{\theta}$ is generated by $\bar{y}_{\theta}$.

Proof. By Theorem 2.2, an element of $e_{\theta} R_{\theta} e_{\theta}$ is a linear combination of terms of the form $\psi_{w} y_{1}^{a_{1}} \ldots y_{d}^{a_{d}} e\left(\boldsymbol{i}_{\theta}\right)$ such that $w \boldsymbol{i}_{\theta}=\boldsymbol{i}_{\theta}$. If $w \in\left(\mathfrak{S}_{\mathrm{ht}\left(\theta_{2}\right)} \times\right.$ $\left.\mathfrak{S}_{\text {ht }\left(\theta_{1}\right)}\right) \backslash \mathfrak{D}_{\theta_{2}, \theta_{1}}$, then $\psi_{w} e_{\theta} \in I_{>(\theta)}$ by Lemmas [5.10] and [5.5. So we may assume that $w=u v$ with $u \in \mathfrak{S}^{\operatorname{ht}\left(\theta_{2}\right), \operatorname{ht}\left(\theta_{1}\right)}, v \in \mathfrak{D}_{\theta_{2}, \theta_{1}}$. It is easy to check that the only such permutation that fixes $\boldsymbol{i}_{\theta}$ is the identity. We therefore see that $\bar{e}_{\theta} \bar{R}_{\theta} \bar{e}_{\theta}$ is generated by $\bar{y}_{1}, \ldots, \bar{y}_{\mathrm{ht}(\theta)}$.

Note that $\operatorname{ht}\left(\theta_{2}\right)=16$ and $\operatorname{ht}\left(\theta_{1}\right)=13$. Using the cases $\beta=\theta_{2}$ and $\beta=\theta_{1}$ proved above and Lemma [5.10, we have that $\left(y_{r}-y_{s}\right) e\left(\boldsymbol{i}_{\theta}\right) \in I_{>(\theta)}$ if $1 \leq$ $r, s \leq 16$ or $17 \leq r, s \leq 29$. It remains to show that $\left(y_{r}-y_{s}\right) e\left(\boldsymbol{i}_{\theta}\right) \in I_{>(\theta)}$ for some $1 \leq r \leq 16$ and $17 \leq s \leq 29$. Let $w \in \mathfrak{S}_{29}$ be the cycle $(27,26, \ldots, 16)$. By considering words and using Corollary 3.3 , one can verify that

$$
\psi_{w}^{\tau} \psi_{w} e\left(\boldsymbol{i}_{\theta}\right) \equiv\left(y_{16}-y_{27}\right) e\left(\boldsymbol{i}_{\theta}\right) \quad\left(\bmod I_{>(\theta)}\right) .
$$


On the other hand, by the formula for the character of $L(\theta)$ from Lemma 5.8 , we have that $w \boldsymbol{i}_{\theta}$ is not a word of $L(\theta)$. Therefore, by Corollary 3.3, we have that $\psi_{w} e\left(\boldsymbol{i}_{\theta}\right) \in I_{>(\theta)}$, so $\left(y_{16}-y_{27}\right) e\left(\boldsymbol{i}_{\theta}\right) \in I_{>(\theta)}$, and we are done.

5.5. Non-symmetric types. Now we deal with non-symmetric Cartan matrices, i.e. Cartan matrices of $B C F G$ types.

Lemma 5.12. Suppose that $\delta_{\beta}, D_{\beta} \in e\left(\boldsymbol{i}_{\beta}\right) R_{\beta} e\left(\boldsymbol{i}_{\beta}\right)$ have been chosen so that Hypothesis 3.9(iii) is satisfied. If the minimal degree component of $e\left(\boldsymbol{i}_{\beta}\right) R_{\beta} e\left(\boldsymbol{i}_{\beta}\right)$ is spanned by $D_{\beta}$, then Hypothesis 3.9(i) and (vi) are satisfied.

Proof. Since $D_{\beta} \delta_{\beta} D_{\beta}$ has the same degree as $D_{\beta}$, the assumption above implies that $D_{\beta} \delta_{\beta} D_{\beta}$ is proportional to $D_{\beta}$. Acting on $v_{\beta}^{+}$and using Hypothesis 3.9(iii) gives $D_{\beta} \delta_{\beta} D_{\beta}=D_{\beta}$, which upon multiplication by $\delta_{\beta}$ on the right gives the property $e_{\beta}^{2}=e_{\beta}$, which is even stronger than (i).

To see (vi), we look at the lowest degree component in $e\left(\boldsymbol{i}_{\beta} \boldsymbol{i}_{\beta}\right) R_{2 \beta} e\left(\boldsymbol{i}_{\beta} \boldsymbol{i}_{\beta}\right)$ using [11, Lemma 5.3(ii)] and commutation relations in the algebra $R_{2 \beta}$.

It will be clear in almost all cases that the condition of Lemma 5.12 will be satisfied, and moreover Hypothesis 3.9(ii) and (iv) are easy to verify by inspection. This leaves Hypothesis 3.9 (v) to be shown in each case.

5.5.1. Type $B_{l}$. The set of positive roots is broken into two types. For $1 \leq i \leq j \leq l$ we have the root $\alpha_{i}+\cdots+\alpha_{j}$, and for $1 \leq i<j \leq l$ we have the root $\alpha_{i}+\cdots+\alpha_{j-1}+2 \alpha_{j}+\cdots+2 \alpha_{l}$.

Let $\beta:=\alpha_{i}+\cdots+\alpha_{j}$. Then $\boldsymbol{i}_{\beta}:=(i, \ldots, j)$, and the irreducible module $L(\beta)$ is one-dimensional with character $\boldsymbol{i}_{\beta}$. Define $\delta_{\beta}:=D_{\beta}:=e\left(\boldsymbol{i}_{\beta}\right)$ and $y_{\beta}:=y_{d} e\left(\boldsymbol{i}_{\beta}\right)$. Using Corollary 3.3 one sees that $\psi_{r} e_{\beta} \in I_{>(\beta)}$ for all $r$, which by Theorem 2.2 shows that $\bar{R}_{\beta} \bar{e}_{\beta}=F\left[\bar{y}_{1}, \ldots, \bar{y}_{d}\right] \bar{e}_{\beta}$. This also shows that for $1 \leq r \leq d$ we have the elements of $I_{>(\beta)}$ :

$$
\psi_{r}^{2} e_{\beta}= \begin{cases}\left(y_{r}-y_{r+1}^{2}\right) e_{\beta}, & \text { if } j=l \text { and } r=d-1 \\ \left(y_{r}-y_{r+1}\right) e_{\beta}, & \text { otherwise. }\end{cases}
$$

It follows that $\bar{R}_{\beta} \bar{e}_{\beta}=F\left[\bar{y}_{\beta}\right] \bar{e}_{\beta}$, and thus $\bar{e}_{\beta} \bar{R}_{\beta} \bar{e}_{\beta}$ is generated by $\bar{e}_{\beta} \bar{y}_{\beta} \bar{e}_{\beta}$.

Consider $\beta:=\alpha_{i}+\cdots+\alpha_{j-1}+2 \alpha_{j}+\cdots+2 \alpha_{l}$. In this case, $\boldsymbol{i}_{\beta}=$ $(i, \ldots, l, l, \ldots, j)$, and $\operatorname{ch}_{q} L(\beta)=\left(q+q^{-1}\right) \boldsymbol{i}_{\beta}$. Define $\delta_{\beta}:=y_{l-i+2} e\left(\boldsymbol{i}_{\beta}\right)$, $D_{\beta}:=\psi_{l-i+1} e\left(\boldsymbol{i}_{\beta}\right)$, and $y_{\beta}=y_{1} e\left(\boldsymbol{i}_{\beta}\right)$. Using Corollary 3.3. one sees that $\psi_{r} e\left(\boldsymbol{i}_{\beta}\right) \in I_{>(\beta)}$ for $r \neq l-i+1$. It is also clear that $\psi_{l-i+1} e_{\beta}=0$, and therefore by Theorem $2.2, \bar{R}_{\beta} \bar{e}_{\beta}=F\left[\bar{y}_{1}, \ldots, \bar{y}_{d}\right] e_{\beta}$. We also have the following elements of $I_{>(\beta)}$ :

$$
\psi_{r}^{2} e\left(\boldsymbol{i}_{\beta}\right)= \begin{cases}\left(y_{r}-y_{r+1}\right) e\left(\boldsymbol{i}_{\beta}\right), & \text { for } 1 \leq r \leq l-i-1 \\ \left(y_{l-i}-y_{l-i+1}^{2}\right) e\left(\boldsymbol{i}_{\beta}\right), & \text { for } r=l-i \\ \left(y_{l-i+3}-y_{l-i+2}^{2}\right) e\left(\boldsymbol{i}_{\beta}\right), & \text { for } r=l-i+2 \\ \left(y_{r+1}-y_{r}\right) e\left(\boldsymbol{i}_{\beta}\right) & \text { for } l-i+3 \leq r \leq d-1 .\end{cases}
$$


Taken together, these show that $\bar{R}_{\beta} \bar{e}\left(\boldsymbol{i}_{\beta}\right)=F\left[\bar{y}_{l-i+1}, \bar{y}_{l-i+2}\right] \bar{e}\left(\boldsymbol{i}_{\beta}\right)$. Multiplying on both sides by $\bar{e}_{\beta}$ and using the KLR / nil-Hecke relations, we have

$$
\bar{e}_{\beta} \bar{R}_{\beta} \bar{e}_{\beta}=F\left[\bar{y}_{l-i+1}+\bar{y}_{l-i+2}, \bar{y}_{l-i+1} \bar{y}_{l-i+2}\right] \bar{e}_{\beta} .
$$

Furthermore, $\left(y_{l-i+1}+y_{l-i+2}\right) \psi_{l-i+1} e\left(\boldsymbol{i}_{\beta}\right)=\psi_{l-i+1} \psi_{l-i}^{2} \psi_{l-i+1} e\left(\boldsymbol{i}_{\beta}\right) \in I_{>(\beta)}$, and so in fact

$$
\bar{e}_{\beta} \bar{R}_{\beta} \bar{e}_{\beta}=F\left[\bar{y}_{l-i+1}^{2}\right] \bar{e}_{\beta}=F\left[\bar{y}_{1}\right] \bar{e}_{\beta} .
$$

5.5.2. Type $C_{l}$. The set of positive roots is broken into three types. For $1 \leq i \leq j \leq l$ we have the root $\alpha_{i}+\cdots+\alpha_{j}$, for $1 \leq i<j<l$ we have the root $\alpha_{i}+\cdots+\alpha_{j-1}+2 \alpha_{j}+\cdots+2 \alpha_{l-1}+\alpha_{l}$, and for $1 \leq i<l$ we have the $\operatorname{root} 2 \alpha_{i}+\cdots+2 \alpha_{l-1}+\alpha_{l}$.

Consider $\beta=\alpha_{i}+\cdots+\alpha_{j}$. Then $\boldsymbol{i}_{\beta}=(i, \ldots, j)$ and $\operatorname{ch}_{q} L(\beta)=\boldsymbol{i}_{\beta}$. Define $\delta_{\beta}=D_{\beta}:=e\left(\boldsymbol{i}_{\beta}\right)$. Define $y_{\beta}:=y_{1} e\left(\boldsymbol{i}_{\beta}\right)$. Using Corollary 3.3 one sees that $\psi_{r} e_{\beta} \in I_{>(\beta)}$ for all $r$, which by Theorem 2.2 shows that $\bar{R}_{\beta} \bar{e}_{\beta}=$ $F\left[\bar{y}_{1}, \ldots, \bar{y}_{d}\right] \bar{e}_{\beta}$. This also shows that for $1 \leq r \leq d$ we have the elements of $I_{>(\beta)}$ :

$$
\psi_{r}^{2} e_{\beta}= \begin{cases}\left(y_{r}^{2}-y_{r+1}\right) e_{\beta}, & \text { if } j=l \text { and } r=d-1 \\ \left(y_{r}-y_{r+1}\right) e_{\beta}, & \text { otherwise. }\end{cases}
$$

Consequently, $\bar{e}_{\beta} \bar{R}_{\beta} \bar{e}_{\beta}$ is generated by $\bar{e}_{\beta} \bar{y}_{\beta} \bar{e}_{\beta}$.

Consider $\beta=\alpha_{i}+\cdots+\alpha_{j-1}+2 \alpha_{j}+\cdots+2 \alpha_{l-1}+\alpha_{l}$. Then $\boldsymbol{i}_{\beta}=(i, \ldots, l-$ $1, l, l-1, \ldots, j)$ and $\operatorname{ch}_{q} L(\beta)=(i, \ldots, l-1, l, l-1, \ldots, j)$. Define $\delta_{\beta}=D_{\beta}:=$ $e\left(\boldsymbol{i}_{\beta}\right)$, and $y_{\beta}:=y_{1} e\left(\boldsymbol{i}_{\beta}\right)$. Using Corollary 3.3 one sees that $\psi_{r} e_{\beta} \in I_{>(\beta)}$ for all $r$, which by Theorem 2.2 shows that $\bar{R}_{\beta} \bar{e}_{\beta}=F\left[\bar{y}_{1}, \ldots, \bar{y}_{d}\right] \bar{e}_{\beta}$. This also shows that for $1 \leq r \leq d$ we have the elements of $I_{>(\beta)}$ :

$$
\psi_{r}^{2} e_{\beta}= \begin{cases}\left(y_{r}-y_{r+1}\right) e_{\beta}, & \text { for } 1 \leq r \leq l-i-1 \\ \left(y_{l-i}^{2}-y_{l-i+1}\right) e_{\beta}, & \text { for } r=l-i \\ \left(y_{l-i+2}^{2}-y_{l-i+1}\right) e_{\beta}, & \text { for } r=l-i+1 \\ \left(y_{r+1}-y_{r}\right) e_{\beta} & \text { for } l-i+2 \leq r \leq d-1\end{cases}
$$

It follows that $\bar{R}_{\beta} \bar{e}_{\beta}=F\left[\bar{y}_{l-i}, \bar{y}_{l-i+2}\right] \bar{e}_{\beta}$. Furthermore, by the relation (2.10),

$$
\left(y_{l-i}+y_{l-i+2}\right) e_{\beta}=\left(\psi_{l-i+1} \psi_{l-i} \psi_{l-i+1}-\psi_{l-i} \psi_{l-i+1} \psi_{l-i}\right) e_{\beta} \in I_{>(\beta)}
$$

and therefore $\bar{R}_{\beta} \bar{e}_{\beta}=F\left[\bar{y}_{l-i}\right] e_{\beta}=F\left[\bar{y}_{\beta}\right] \bar{e}_{\beta}$.

Consider $\beta=2 \alpha_{i}+\cdots+2 \alpha_{l-1}+\alpha_{l}$. Then $\boldsymbol{i}_{\beta}=(i, \ldots, l-1, i, \ldots, l)$ and

$$
\operatorname{ch}_{q} L(\beta)=q((i, \ldots, l-1) \circ(i, \ldots, l-1)) \cdot(l) .
$$

Let $w \in \mathfrak{S}_{d}$ be the permutation that sends $(1,2, \ldots, d)$ to $(l-i+1, \ldots, d-$ $1,1, \ldots, l-i, d)$, and define $D_{\beta}:=\psi_{w} e\left(\boldsymbol{i}_{\beta}\right)$. Define also $\delta_{\beta}:=y_{d-1} e\left(\boldsymbol{i}_{\beta}\right)$ and $y_{\beta}:=y_{d} e\left(\boldsymbol{i}_{\beta}\right)$. Set $\gamma=\alpha_{i}+\cdots+\alpha_{l-1}$. Since $I_{>\left(\gamma^{2}\right)}$ is generated by idempotents $e(\boldsymbol{i})$ with $\boldsymbol{i}>\boldsymbol{i}_{\gamma}^{2}$, and $\boldsymbol{i}_{\beta}=\boldsymbol{i}_{\gamma}^{2} i_{l}$ is the highest weight of $L_{\beta}$, we see that

$$
\iota_{2 \gamma, \alpha_{l}}\left(I_{>\left(\gamma^{2}\right)} \otimes R_{\alpha_{l}}\right) \subseteq I_{>(\beta)} .
$$


Let $\mu: \bar{R}_{2 \gamma} \otimes R_{\alpha_{l}} \rightarrow \bar{R}_{\beta}$ be the induced map. Note that every weight of $L(\beta)$ ends with $l$, so that $\psi_{u} e\left(\boldsymbol{i}_{\beta}\right) \in I_{>(\beta)}$ unless $u \in \mathfrak{S}_{d-1,1}$, by Corollary 3.3. Therefore, applying (3.14) in the type $A$ case of $\left(\gamma^{2}\right)$ (which has already been verified), we obtain

$$
\bar{e}_{\beta} \bar{R}_{\beta} \bar{e}_{\beta}=\mu\left(\bar{e}_{\left(\gamma^{2}\right)} \bar{R}_{2 \gamma} \bar{e}_{\left(\gamma^{2}\right)} \otimes R_{\alpha_{l}}\right)=\bar{e}_{\beta} \mathcal{O}\left[\bar{y}_{l-i}+\bar{y}_{2 l-2 i}, \bar{y}_{l-i} \bar{y}_{2 l-2 i}, \bar{y}_{d}\right] \bar{e}_{\beta} .
$$

Furthermore,

$$
\left(\bar{y}_{l-i}+\bar{y}_{2 l-2 i}\right) \bar{e}\left(\boldsymbol{i}_{\beta}\right)=\bar{\psi}_{l-i} \ldots \bar{\psi}_{2 l-2 i-1} \bar{\psi}_{2 l-2 i}^{2} \bar{\psi}_{2 l-2 i-1} \ldots \bar{\psi}_{l-i} \bar{e}\left(\boldsymbol{i}_{\beta}\right)=0
$$

and $\left(\bar{y}_{2 l-2 i}^{2}-\bar{y}_{d}\right) \bar{e}\left(\boldsymbol{i}_{\beta}\right)=\bar{\psi}_{2 l-2 i}^{2} \bar{e}\left(\boldsymbol{i}_{\beta}\right)=0$. Thus $\bar{e}_{\beta} \bar{R}_{\beta} \bar{e}_{\beta}$ is generated by $\bar{e}_{\beta} \bar{y}_{d} \bar{e}_{\beta}$.

5.5.3. Type $F_{4}$. We write $\beta=c_{1} \alpha_{1}+c_{2} \alpha_{2}+c_{3} \alpha_{3}+c_{4} \alpha_{4} \in \Phi_{+}$. If $c_{4}=0$, then this root lies in a subsystem of type $B_{3}$ with the same order as in section 5.5 .1 and we are done.

If $\beta=\alpha_{i}+\cdots+\alpha_{j}$ for some $1 \leq i \leq j \leq 4$, then $\boldsymbol{i}_{\beta}=(i, \ldots, j)$ and $\operatorname{ch}_{q} L(\beta)=(i, \ldots, j)$. In this case we take $D_{\beta}=\delta_{\beta}=e\left(\boldsymbol{i}_{\beta}\right)$, and set $y_{\beta}=y_{\mathrm{ht}(\beta)} e\left(\boldsymbol{i}_{\beta}\right)$.

The following table shows the choice of data for the remaining roots, except for the highest root $\beta=2 \alpha_{1}+3 \alpha_{2}+4 \alpha_{3}+2 \alpha_{4}$, which we discuss separately. In each of these cases, the hypotheses may be verified by employing the same methods used above. For example, in each case either Hypothesis 3.9(i)-(iv),(vi) may be verified directly or with the help of Lemma 5.12 when it applies.

\begin{tabular}{|c|c|c|c|}
\hline $\boldsymbol{i}_{\beta}$ & $D_{\beta}$ & $\delta_{\beta}$ & $y_{\beta}$ \\
\hline 2343 & $e\left(\boldsymbol{i}_{\beta}\right)$ & $e\left(\boldsymbol{i}_{\beta}\right)$ & $y_{3} e\left(\boldsymbol{i}_{\beta}\right)$ \\
12343 & $e\left(\boldsymbol{i}_{\beta}\right)$ & $e\left(\boldsymbol{i}_{\beta}\right)$ & $y_{5} e\left(\boldsymbol{i}_{\beta}\right)$ \\
23434 & $\psi_{3} \psi_{2} \psi_{4} \psi_{3} e\left(\boldsymbol{i}_{\beta}\right)$ & $y_{5} e\left(\boldsymbol{i}_{\beta}\right)$ & $y_{1} e\left(\boldsymbol{i}_{\beta}\right)$ \\
123432 & $e\left(\boldsymbol{i}_{\beta}\right)$ & $e\left(\boldsymbol{i}_{\beta}\right)$ & $y_{5} e\left(\boldsymbol{i}_{\beta}\right)$ \\
123434 & $\psi_{4} \psi_{3} \psi_{5} \psi_{4} e\left(\boldsymbol{i}_{\beta}\right)$ & $y_{6} e\left(\boldsymbol{i}_{\beta}\right)$ & $y_{2} e\left(\boldsymbol{i}_{\beta}\right)$ \\
1234323 & $e\left(\boldsymbol{i}_{\beta}\right)$ & $e\left(\boldsymbol{i}_{\beta}\right)$ & $y_{5} e\left(\boldsymbol{i}_{\beta}\right)$ \\
1234342 & $\psi_{4} \psi_{3} \psi_{5} \psi_{4} e\left(\boldsymbol{i}_{\beta}\right)$ & $y_{6} e\left(\boldsymbol{i}_{\beta}\right)$ & $y_{2} e\left(\boldsymbol{i}_{\beta}\right)$ \\
12343423 & $\psi_{4} \psi_{3} \psi_{5} \psi_{4} e\left(\boldsymbol{i}_{\beta}\right)$ & $y_{6} e\left(\boldsymbol{i}_{\beta}\right)$ & $y_{8} e\left(\boldsymbol{i}_{\beta}\right)$ \\
123434233 & $\psi_{4} \psi_{3} \psi_{5} \psi_{4} \psi_{8} e\left(\boldsymbol{i}_{\beta}\right)$ & $y_{6} y_{9} e\left(\boldsymbol{i}_{\beta}\right)$ & $y_{7} e\left(\boldsymbol{i}_{\beta}\right)$ \\
1234342332 & $\psi_{4} \psi_{3} \psi_{5} \psi_{4} \psi_{8} e\left(\boldsymbol{i}_{\beta}\right)$ & $y_{6} y_{9} e\left(\boldsymbol{i}_{\beta}\right)$ & $y_{10} e\left(\boldsymbol{i}_{\beta}\right)$ \\
\hline
\end{tabular}

Consider now $\beta=2 \alpha_{1}+3 \alpha_{2}+4 \alpha_{3}+2 \alpha_{4}$, where $\boldsymbol{i}_{\beta}=(12343123432)$. Let $w \in \mathfrak{S}_{11}$ be the permutation that sends $(1, \ldots, 11)$ to $(6,7,8,9,10,1,2,3,4,5,11)$, and set $D_{\beta}=\psi_{w} e\left(\boldsymbol{i}_{\beta}\right)$. Let $\delta_{\beta}=y_{10} e\left(\boldsymbol{i}_{\beta}\right)$, and $y_{\beta}=y_{11} e\left(\boldsymbol{i}_{\beta}\right)$. Define $\gamma=\alpha_{1}+\alpha_{2}+2 \alpha_{3}+\alpha_{4}$. There is a map $\mu: \bar{R}_{2 \gamma} \otimes R_{\alpha_{2}} \rightarrow \bar{R}_{\beta}$. This map is not surjective, but one can show that

$$
\bar{e}\left(\boldsymbol{i}_{\beta}\right) \bar{R}_{\beta} \bar{e}\left(\boldsymbol{i}_{\beta}\right)=\mu\left(\bar{e}\left(\boldsymbol{i}_{\gamma}^{2}\right) \bar{R}_{2 \gamma} \bar{e}\left(\boldsymbol{i}_{\gamma}^{2}\right) \otimes R_{\alpha_{2}}\right)
$$

and thus

$$
\begin{aligned}
\bar{e}_{\beta} \bar{R}_{\beta} \bar{e}_{\beta} & =\mu\left(\bar{e}_{\left(\gamma^{2}\right)} \bar{R}_{2 \gamma} \bar{e}_{\left(\gamma^{2}\right)} \otimes R_{\alpha_{2}}\right)=\mu\left(\mathcal{O}\left[\bar{y}_{5}+\bar{y}_{10}, \bar{y}_{5} \bar{y}_{10}\right] \bar{e}_{\left(\gamma^{2}\right)} \otimes R_{\alpha_{2}}\right) \\
& =\mathcal{O}\left[\bar{y}_{5}+\bar{y}_{10}, \bar{y}_{5} \bar{y}_{10}, \bar{y}_{11}\right] \bar{e}_{\beta} .
\end{aligned}
$$


We also compute (cf. [19, §5]):

$$
\left(\bar{y}_{5}+\bar{y}_{10}\right) \bar{e}\left(\boldsymbol{i}_{\beta}\right)=-\bar{\psi}_{5} \bar{\psi}_{6} \bar{\psi}_{7} \bar{\psi}_{8} \bar{\psi}_{9} \bar{\psi}_{10}^{2} \bar{\psi}_{9} \bar{\psi}_{8} \bar{\psi}_{7} \bar{\psi}_{6} \bar{\psi}_{5} \bar{e}\left(\boldsymbol{i}_{\beta}\right)
$$

which is zero because it contains the weight (12341234323), and this is not a weight of $L(\beta)$. Since $\bar{y}_{11} \bar{e}\left(\boldsymbol{i}_{\beta}\right)=\bar{y}_{10}^{2} \bar{e}\left(\boldsymbol{i}_{\beta}\right)$, we see that $\bar{e}_{\beta} \bar{R}_{\beta} \bar{e}_{\beta}=\mathcal{O}\left[\bar{y}_{11}\right] \bar{e}_{\beta}$, as required.

5.5.4. Type $G_{2} \cdot \beta=\alpha_{1}: \boldsymbol{i}_{\beta}=(1), D_{\beta}=\delta_{\beta}=e\left(\boldsymbol{i}_{\beta}\right)$, and $y_{\beta}=y_{1} e\left(\boldsymbol{i}_{\beta}\right)$.

$\beta=\alpha_{2}: \boldsymbol{i}_{\beta}=(2), D_{\beta}=\delta_{\beta}=e\left(\boldsymbol{i}_{\beta}\right)$, and $y_{\beta}=y_{1} e\left(\boldsymbol{i}_{\beta}\right)$.

$\beta=\alpha_{1}+\alpha_{2}: \boldsymbol{i}_{\beta}=(12), D_{\beta}=\delta_{\beta}=e\left(\boldsymbol{i}_{\beta}\right)$, and $y_{\beta}=y_{1} e\left(\boldsymbol{i}_{\beta}\right)$.

$\beta=2 \alpha_{1}+\alpha_{2}: \boldsymbol{i}_{\beta}=(112), D_{\beta}=\psi_{1} e\left(\boldsymbol{i}_{\beta}\right), \delta_{\beta}=y_{2} e\left(\boldsymbol{i}_{\beta}\right)$, and $y_{\beta}=$ $\left(y_{1}+y_{2}\right) e\left(\boldsymbol{i}_{\beta}\right)$.

$\beta=3 \alpha_{1}+\alpha_{2}: \boldsymbol{i}_{\beta}=(1112), D_{\beta}=\psi_{1} \psi_{2} \psi_{1} e\left(\boldsymbol{i}_{\beta}\right), \delta_{\beta}=y_{2} y_{3}^{2} e\left(\boldsymbol{i}_{\beta}\right)$, and $y_{\beta}=y_{1} y_{2} y_{3} e\left(\boldsymbol{i}_{\beta}\right)$.

Let $\mu$ be the composition $R_{3 \alpha_{1}} \otimes R_{\alpha_{2}} \hookrightarrow R_{\beta} \rightarrow \bar{R}_{\beta}$. If $w \notin \mathfrak{S}_{3,1}$, then $\psi_{w} e\left(\boldsymbol{i}_{\beta}\right) \in I_{>(\beta)}$, and so $\mu$ is surjective. Furthermore, $\bar{e}_{\beta}=\mu\left(e_{\left(\alpha_{1}^{3}\right)} \otimes 1\right)$. Thus $\bar{e}_{\beta} \bar{R}_{\beta} \bar{e}_{\beta}=\mathcal{O}\left[\bar{y}_{1}, \bar{y}_{2}, \bar{y}_{3}, \bar{y}_{4}\right]^{\mathfrak{S}_{3,1}} \bar{e}_{\beta}$. Since $\left(\bar{y}_{3}^{3}-\bar{y}_{4}\right) \bar{e}\left(\boldsymbol{i}_{\beta}\right)=\bar{\psi}_{3}^{2} \bar{e}\left(\boldsymbol{i}_{\beta}\right)=0$, we have that $\bar{e}_{\beta} \bar{R}_{\beta} \bar{e}_{\beta}$ is generated by $\mathcal{O}\left[\bar{y}_{1}, \bar{y}_{2}, \bar{y}_{3}\right]^{\mathfrak{S}_{3}} \bar{e}_{\beta}$. Observe using [10, Theorem $4.12(\mathrm{i})]$ that

$$
\left(\bar{y}_{1}+\bar{y}_{2}+\bar{y}_{3}\right) \bar{e}_{\beta}=\bar{e}_{\beta} \bar{\psi}_{1} \bar{\psi}_{2} \bar{\psi}_{3}^{2} \bar{e}_{\beta} \in I_{>(\beta)}
$$

and

$$
\left(\left(\bar{y}_{1}+\bar{y}_{2}+\bar{y}_{3}\right)^{2}-\left(\bar{y}_{1} \bar{y}_{2}+\bar{y}_{1} \bar{y}_{3}+\bar{y}_{2} \bar{y}_{3}\right)\right) \bar{e}_{\beta}=\bar{e}_{\beta} \bar{\psi}_{2} \bar{\psi}_{3}^{2} \bar{e}_{\beta} \in I_{>(\beta)} .
$$

Therefore $\bar{e}_{\beta} \bar{R}_{\beta} \bar{e}_{\beta}$ is generated by $\bar{y}_{1} \bar{y}_{2} \bar{y}_{3}$.

$\beta=3 \alpha_{1}+2 \alpha_{2}: \boldsymbol{i}_{\beta}=(11212), D_{\beta}=\psi_{1} \psi_{3} \psi_{2} \psi_{4} \psi_{1} \psi_{3} e\left(\boldsymbol{i}_{\beta}\right), \delta_{\beta}=y_{2} y_{4}^{2} e\left(\boldsymbol{i}_{\beta}\right)$, and $y_{\beta}=y_{1} y_{2} y_{4} e\left(\boldsymbol{i}_{\beta}\right)$. We first prove

Claim: If $w \neq 1$ then $e\left(\boldsymbol{i}_{\beta}\right) \psi_{w} e_{\beta} \in I_{>(\beta)}$.

This is clearly true unless $w$ is one of the twelve permutations that stabilizes the weight $\boldsymbol{i}_{\beta}$. Of these, six produce a negative degree. Since $D_{\beta}$ spans the smallest degree component of $e\left(\boldsymbol{i}_{\beta}\right) R_{\beta} e\left(\boldsymbol{i}_{\beta}\right)$ we the Claim holds for these six permutations. Two of the remaining six permutations end with the cycle (12). Since $\psi_{1} D_{\beta}=0$, this implies that the Claim holds for them too. Finally, reduced decompositions for the remaining non-identity permutations may be chosen so that $e\left(\boldsymbol{i}_{\beta}\right) \psi_{w} \in I_{>(\beta)}$ by Lemmas 3.2 and 5.4 .

Now we combine the Claim with Theorem 2.2 to see that $\bar{e}_{\beta} \bar{R}_{\beta} \bar{e}_{\beta}$ is generated by $\bar{e}_{\beta} \mathcal{O}\left[\bar{y}_{1}, \ldots, \bar{y}_{5}\right] \bar{e}_{\beta}$. Next, by weights and quadratic relations, $\left(y_{3}-y_{2}^{3}\right) e\left(\boldsymbol{i}_{\beta}\right),\left(y_{5}-y_{4}^{3}\right) e\left(\boldsymbol{i}_{\beta}\right) \in I_{>(\beta)}$. Thus $\bar{e}_{\beta} \bar{R}_{\beta} \bar{e}_{\beta}=\bar{e}_{\beta} \mathcal{O}\left[\bar{y}_{1}, \bar{y}_{2}, \bar{y}_{4}\right] \bar{e}_{\beta}$. This can then be seen to be equal to $\mathcal{O}\left[\bar{y}_{1}, \bar{y}_{2}, \bar{y}_{4}\right]^{\mathfrak{S}_{3}} \bar{e}_{\beta}$, arguing as in the case of the root $3 \alpha_{1}+\alpha_{2}$ above. Again as in the case of the root $3 \alpha_{1}+\alpha_{2}$, one then shows using specific elements of $I_{>(\beta)}$ that $\left(\bar{y}_{1}+\bar{y}_{2}+\bar{y}_{4}\right) \bar{e}_{\beta}=0$ and $\left(\bar{y}_{1} \bar{y}_{2}+\bar{y}_{1} \bar{y}_{4}+\bar{y}_{2} \bar{y}_{4}\right) \bar{e}_{\beta}=0$, so that $\bar{e}_{\beta} \bar{R}_{\beta} \bar{e}_{\beta}=\mathcal{O}\left[\bar{y}_{1} \bar{y}_{2} \bar{y}_{4}\right] e_{\beta}$. 


\section{REFERENCES}

[1] J. Brundan and A. Kleshchev, Graded decomposition numbers for cyclotomic Hecke algebras, Adv. Math. 222 (2009), 1883-1942.

[2] J. Brundan, A. Kleshchev and P.J. McNamara, Homological properties of finite type Khovanov-Lauda-Rouquier algebras, Duke Math. J., to appear; arXiv: 1210.6900

[3] G. Benkart, S.-J. Kang, S.-J Oh, and E.Park, Construction of irreducible representations over Khovanov-Lauda-Rouquier algebras of finite classical type, IMRN, to appear; arXiv:1108.1048.

[4] D. Hill, G. Melvin, and D. Mondragon, Representations of quiver Hecke algebras via Lyndon bases, J. Pure Appl. Algebra 216 (2012), 1052-1079.

[5] V. G. Kac, Infinite Dimensional Lie Algebras, Cambridge University Press, 1990.

[6] S. Kato, PBW bases and KLR algebras, arXiv:1203.5254.

[7] M. Khovanov and A. Lauda, A diagrammatic approach to categorification of quantum groups I, Represent. Theory 13 (2009), 309-347.

[8] M. Khovanov and A. Lauda, A diagrammatic approach to categorification of quantum groups II, Trans. Amer. Math. Soc. 363 (2011), 2685-2700.

[9] A. Kleshchev, Cuspidal systems for affine Khovanov-Lauda-Rouquier algebras, Math. Z., to appear; arXiv:1210.6556.

[10] A. Kleshchev, J. Loubert, and V. Miemietz, Affine Cellularity of Khovanov-LaudaRouquier algebras in type A, J. Lond. Math. Soc., to appear; $\operatorname{arXiv:1210.6542}$

[11] A. Kleshchev and A. Ram, Representations of Khovanov-Lauda-Rouquier algebras and combinatorics of Lyndon words, Math. Ann. 349 (2011), no. 4, 943-975.

[12] A. Kleshchev and A. Ram, Homogeneous representations of KhovanovLauda algebras, J. Eur. Math. Soc. 12 (2010), 1293-1306.

[13] S. Koenig and A. Kleshchev, Affine highest weight categories and affine quasihereditary algebras, preprint, 2013.

[14] S. Koenig and C. Xi, Affine cellular algebras, Adv. Math. 229 (2012), no. 1, 139 182.

[15] A.D. Lauda and M. Vazirani, Crystals from categorified quantum groups, Adv. Math. 228(2011), 803-861.

[16] B. Leclerc, Dual canonical bases, quantum shuffles and q-characters, Math. Z. 246 (2004), 691-732.

[17] G. Lusztig, Introduction to Quantum Groups, Progress in mathematics 110, Birkhäuser, Boston, 1993.

[18] L. Manivel, Symmetric functions, Schubert polynomials and Degeneracy Loci, vol. 6 of SMF/AMS Texts and Monographs. AMS, Providence, RI, 2001.

[19] P.J. McNamara, Finite dimensional representations of Khovanov-Lauda-Rouquier algebras I: finite type, J. reine angew. Math., to appear; arXiv:1207.5860.

[20] R. Rouquier, 2-Kac-Moody algebras; arXiv:0812.5023

Department of Mathematics, University of Oregon, Eugene, OR 97403, USA

E-mail address: klesh@uoregon.edu

Department of Mathematics, University of Oregon, Eugene, OR 97403, USA

E-mail address: loubert@uoregon.edu 